
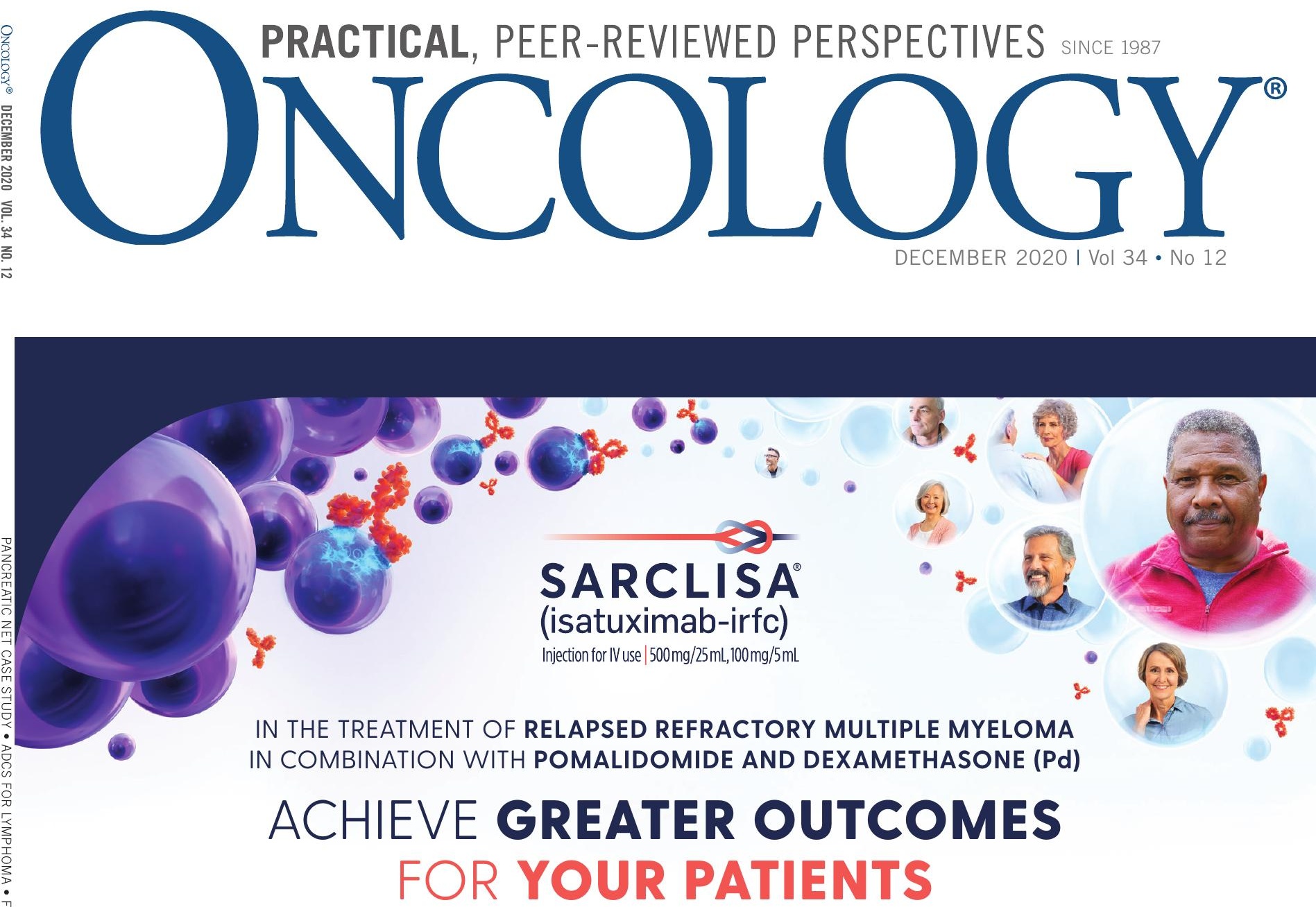

SARCLISA is an anti-CD38 therapy proven to deliver superior PFS (median PFS, 11.53 months with SARCLISA + Pd vs 6.47 months with Pd alone, $\mathrm{HR}=0.596,95 \% \mathrm{Cl}$ : $0.44,0.81, P=0.0010)$. SARCLISA also demonstrated a significant increase in ORR $(60.4 \%$ with SARCLISA + Pd

[95\% Cl: $52.2 \%, 68.2 \%]$ vs 35.3\% with Pd alone [95\% Cl: $27.8 \%, 43.4 \%], P<0.0001)^{1 *}$

*ORR included SCR, CR, VGPR, and PR. SCR, CR, VGPR, and PR were evaluated by an IRC using the IMWG response criteria.

$\mathrm{CR}=$ complete response; IMWG=International Myeloma Working Group; IRC=independent response committee; ORR=overall response rate; $\mathrm{PFS}=$ progression-free survival; $\mathrm{PR}=$ partial response; $\mathrm{s} C \mathrm{R}=$ stringent complete response; VGPR=very good partial response.

\section{Indication}

SARCLISA (isatuximab-irfc) is indicated, in combination with pomalidomide and dexamethasone, for the treatment of adult patients with multiple myeloma who have received at least two prior therapies including lenalidomide and a proteasome inhibitor.

\section{Important Safety Information}

\section{CONTRAINDICATIONS}

SARCLISA is contraindicated in patients with severe hypersensitivity to isatuximab-irfc or to any of its excipients.

Please see Important Safety Information throughout, and accompanying Brief Summary of the full Prescribing Information.

\section{WARNINGS AND PRECAUTIONS Infusion-Related Reactions}

Infusion-related reactions (IRRs) have been observed in $39 \%$ of patients treated with SARCLISA. All IRRs started during the first SARCLISA infusion and resolved on the same day in $98 \%$ of the cases. The most common symptoms of an IRR included dyspnea, cough, chills, and nausea. The most common severe signs and symptoms included hypertension and dyspnea. 
SARCLISA Is the First Anti-CD38 Antibody Studied.in a Phase 3 Trial in Combination With Pd vs Pd Alone

- ICARIA-MM: A multicenter, open-label, randomized, phase 3 study? -

Patients with relapsed refractory multiple myeloma who received at least 2 prior therapies, including lenalidomide and a PI (N=307)

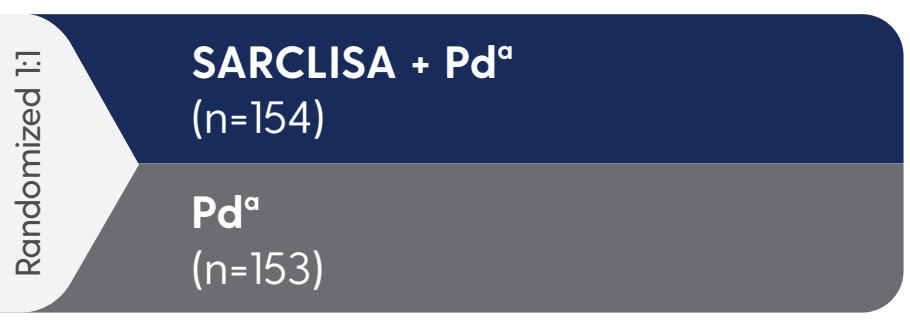

aPomalidomide $4 \mathrm{mg}$ was taken orally once daily from day 1 to day 21 of each 28 -day cycle. Low-dose dexamethasone (orally or IV) $40 \mathrm{mg}$ (20 mg for patients $\geq 75$ years of age) was given on days 1, 8, 15, and 22 for each 28-day cycle.

- SARCLISA $10 \mathrm{mg} / \mathrm{kg}$ was administered as an IV infusion weekly in the first cycle and every 2 weeks thereafter

- Treatment administered in 28-day cycles until disease progression or unacceptable toxicity

Primary endpoint: PFS*

Key secondary endpoints: ORR, ${ }^{\dagger}$ OS

The phase 3 ICARIA-MM trial included patients with poor prognostic factors ${ }^{1-3}$

Treatment history

$93 \%$ Refractory to lenalidomide

$73 \%$ Refractory to IMiD ${ }^{\oplus}+\mathrm{PI}$

$56 \%$ Received prior ASCT

\section{Patient factors}

\section{$36 \%$ Impaired renal function}

\section{$20 \%$}

High-risk chromosomal abnormalities

\section{$20 \% \geq 75$ years}

*PFS results were assessed by an IRC, based on central laboratory data for M-protein, and central radiologic imaging review using the IMWG criteria. Median time to follow-up was 11.6 months.

${ }_{\mathrm{I}} \mathrm{CR}, \mathrm{CR}$, VGPR, and PR were evaluated by the IRC using the IMWG response criteria.

ASCT=autologous stem cell transplant; IMiD=immunomodulatory drug; IV=intravenous; M-protein=myeloma protein; OS=overall survival; $\mathrm{Pl}=$ proteasome inhibitor.

\section{Important Safety Information (cont'd)}

Infusion-Related Reactions (cont'd)

To decrease the risk and severity of IRRs, premedicate patients prior to SARCLISA infusion with acetaminophen, $\mathrm{H}_{2}$ antagonists, diphenhydramine or equivalent, and dexamethasone. Monitor vital signs frequently during the entire SARCLISA infusion. For patients with grade 1 or 2 reactions, interrupt SARCLISA infusion and provide appropriate medical support.

Please see Important Safety Information throughout, and accompanying Brief Summary of the full Prescribing Information. 


\section{SARCLISA + Pd Extended Median PFS to 1 Year}

\section{Superior PFS with SARCLISA + Pd vs Pd alone'}

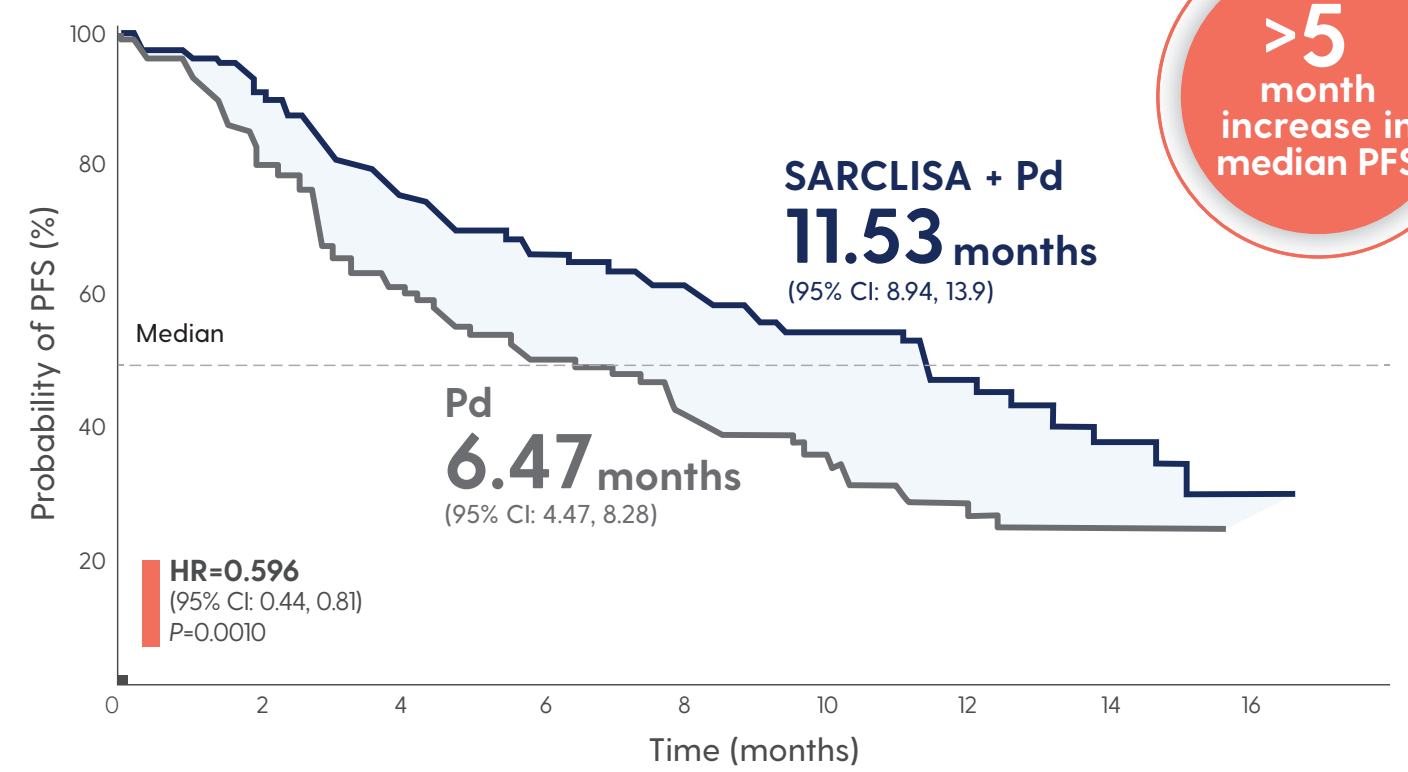

Patients at risk

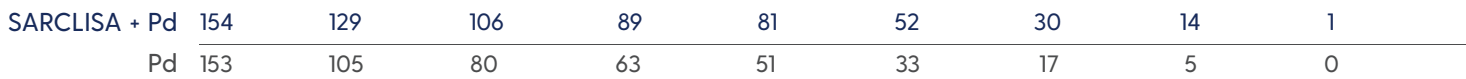

The median duration of treatment was 41 weeks with SARCLISA + Pd vs 24 weeks with Pd.' At a median follow-up time of 11.6 months, 43 patients $(27.9 \%)$ receiving SARCLISA + Pd and 56 patients (36.6\%) receiving Pd had died. Median OS was not reached for either treatment group at interim analysis. The OS results at interim analysis did not reach statistical significance.'

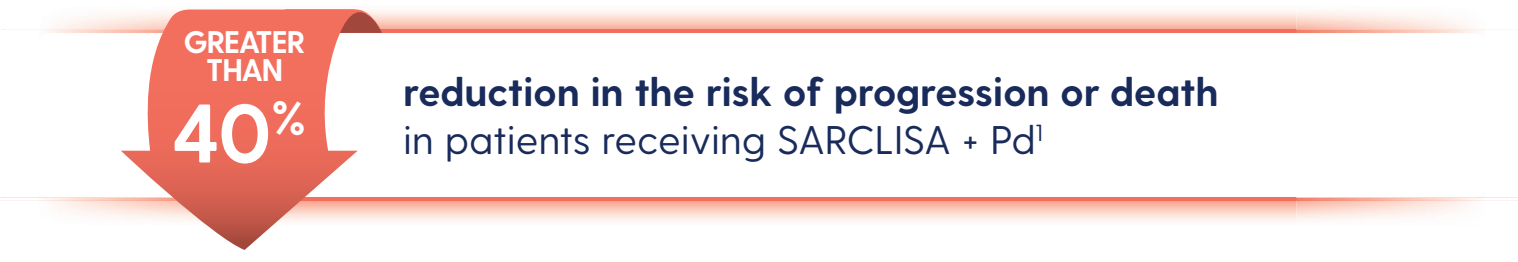

\section{Important Safety Information (cont'd)}

\section{Infusion-Related Reactions (cont'd)}

If symptoms improve, restart SARCLISA infusion at half of the initial rate, with supportive care as needed, and closely monitor patients. If symptoms do not recur after 30 minutes, the infusion rate may be increased to the initial rate, and then increased incrementally. In case symptoms do not improve or recur after interruption, permanently discontinue SARCLISA and institute appropriate management. Permanently discontinue SARCLISA if a grade 3 or higher IRR occurs and institute appropriate emergency medical management. 


\section{SARCLISA + Pd Showed a Significant Increase in ORR}

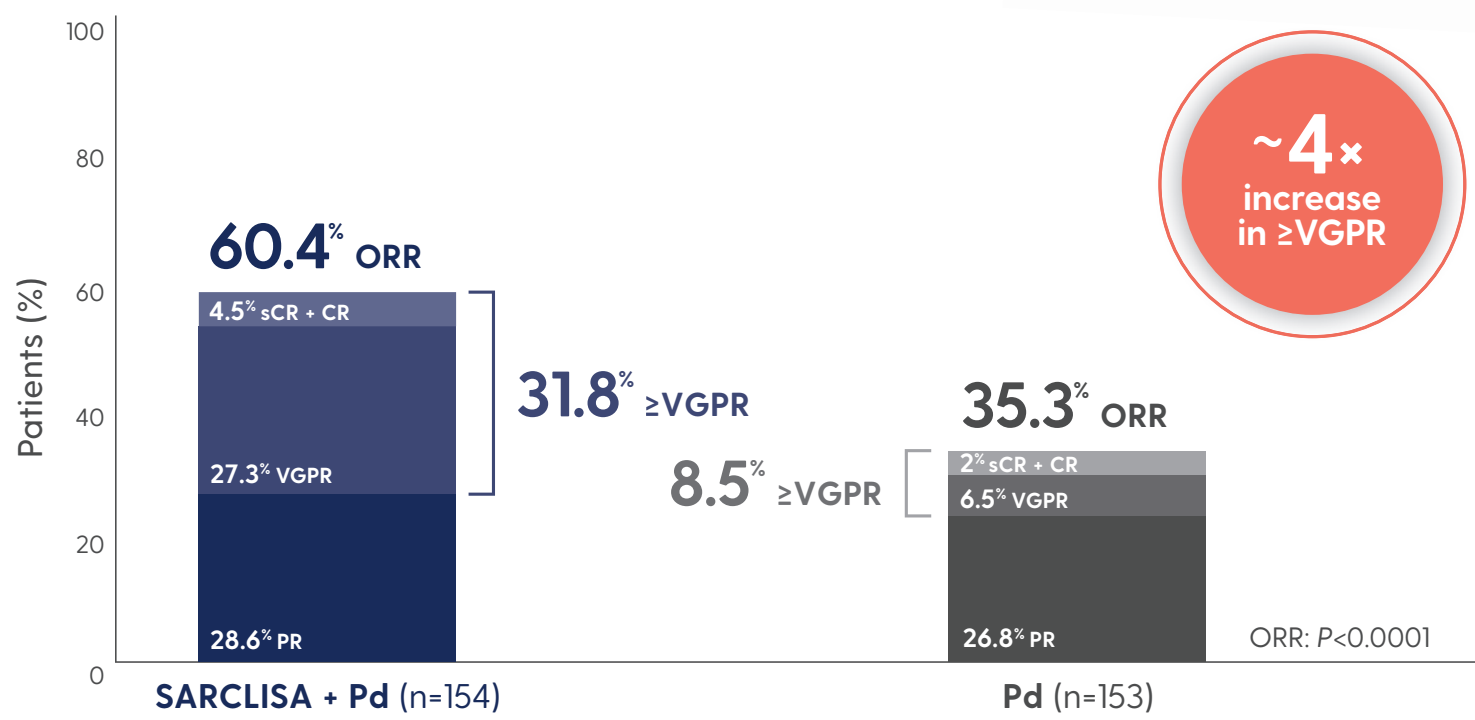

ORR: SARCLISA + Pd (95\% Cl: 52.2\%, 68.2\%), Pd (95\% Cl: 27.8\%, 43.4\%). 95\% Cl estimated using the Clopper-Pearson method.

The median duration of response among responders was 13.3 months (95\% Cl: 10.6, NR) with SARCLISA + Pd vs 11.1 months $(95 \% \mathrm{Cl}: 8.5, \mathrm{NR})$ with Pd alone.?

\section{NCCN Clinical Practice Guidelines in Oncology (NCCN Guidelines ${ }^{\circledR}$ )}

\section{Preferred Category 1 recommendation for isatuximab-irfc (SARCLISA)}

Isatuximab-irfc (SARCLISA), in combination with pomalidomide and dexamethasone, is a Preferred Category 1 option for previously treated multiple myeloma by the National Comprehensive Cancer Network ${ }^{\oplus}\left(\mathrm{NCCN}^{\oplus}\right){ }^{4}$

NCCN makes no warranties of any kind whatsoever regarding their content, use or application and disclaims any responsibility for their application or use in any way.

NR=not reached

\section{Important Safety Information (cont'd)}

\section{Neutropenia}

SARCLISA may cause neutropenia. Neutropenia (reported as laboratory abnormality) occurred in $96 \%$ of patients and grade $3-4$ neutropenia occurred in $85 \%$ of patients treated with SARCLISA, pomalidomide, and dexamethasone (Isa-Pd). Febrile neutropenia occurred in $12 \%$ of patients and neutropenic infections, defined as infection with concurrent grade $\geq 3$ neutropenia, occurred in $25 \%$ of patients treated with Isa-Pd. The most frequent neutropenic infections included those of upper respiratory tract $(10 \%)$, lower respiratory tract $(9 \%)$, and urinary tract $(3 \%)$. 
SARCLISA

Rx Only

(isatuximab-irfc) injection, for intravenous use Brief Summary of Prescribing Information 1 INDICATIONS AND USAGE

SARCLISA is indicated, in combination with pomalidomide and dexamethasone, for the treatment of adult patients with multiple myeloma who have received at least two prior therapies including lenalidomide and a proteasome inhibitor. 2 DOSAGE AND ADMINISTRATION

2.1 Recommended Dosage

- Administer pre-infusion medications [see Dosage and

Administration (2.2)].

- SARCLISA should be administered by a healthcare professional, with immediate access to emergency equipment and appropriate medical support to manage infusion-related reactions if they occur [see Warnings and Precautions (5.1)].

The recommended dose of SARCLISA is $10 \mathrm{mg} / \mathrm{kg}$ actual body weight administered as an intravenous infusion in combination with pomalidomide and dexamethasone, according to the schedule in Table 1 [see Clinical Studies (14) in the full prescribing information]

Table 1: SARCLISA Dosing Schedule in Combination with Pomalidomide and Dexamethasone

\begin{tabular}{|l|l|}
\hline Cycle & Dosing schedule \\
\hline Cycle 1 & Days 1, 8, 15, and 22 (weekly) \\
\hline Cycle 2 and beyond & Days 1, 15 (every 2 weeks) \\
\hline
\end{tabular}

Each treatment cycle consists of a 28-day period. Treatment is repeated until disease progression or unacceptable toxicity. SARCLISA is used in combination with pomalidomide an dexamethasone.

Missed SARCLISA Doses

If a planned dose of SARCLISA is missed, administer the dose as soon as possible and adjust the treatment schedule accordingly, maintaining the treatment interval.

\section{Recommended Premedications}

Administer the following premedications prior to SARCLISA infusion to reduce the risk and severity of infusion-related reactions [see Warnings and Precautions (5.1)].

- Dexamethasone $40 \mathrm{mg}$ orally or intravenously (or $20 \mathrm{mg}$

orally or intravenously for patients $\geq 75$ years of age).

- Acetaminophen $650 \mathrm{mg}$ to $1000 \mathrm{mg}$ orally (or equivalent).

- H2 antagonists.

- Diphenhydramine $25 \mathrm{mg}$ to $50 \mathrm{mg}$ orally or intravenously

(or equivalent). The intravenous route is preferred for at

least the first 4 infusions.

The above recommended dose of dexamethasone (orally

or intravenously) corresponds to the total dose to be

administered only once before infusion as part of the

premedication and of the backbone treatment, before

SARCLISA and pomalidomide administration.

Administer the recommended premedication agents 15 to 60

minutes prior to starting a SARCLISA infusion.

2.3 Dose Modifications

No dose reduction of SARCLISA is recommended. Dose delay may be required to allow recovery of blood counts in the event of hematological toxicity [see Warnings and Precautions (5.2, 5.4)] For information concerning drugs given in combination with SARCLISA, see manufacturer's prescribing information. For other medicinal products that are administered with

SARCLISA, refer to the respective current prescribing information.

2.4 Preparation

Prepare the solution for infusion using aseptic technique as follows: Calculate the dose (mg) of required SARCLISA based on

actual patient weight (measured prior to each cycle to have

the administered dose adjusted accordingly) (see Dosage an Administration (2.1)]. More than one SARCLISA vial may be necessary to obtain the required dose for the patient.

- Parenteral drug products should be inspected visually for

particulate matter and discoloration prior to administration, whenever solution and container permit.

- Remove the volume of diluent from the $250 \mathrm{~mL}$ Sodium

Chloride Injection, USP, or 5\% Dextrose Injection,

USP diluent bag that is equal to the required volume of

SARCLISA injection.

- Withdraw the necessary volume of SARCLISA injection and dilute by adding to the infusion bag of $0.9 \%$ Sodium

Chloride Injection, USP or $5 \%$ Dextrose Injection, USP

to achieve the appropriate SARCLISA concentration for infusion.

- The infusion bag must be made of polyolefins (PO), polyethylene (PE), polypropylene (PP), polyvinyl chloride (PVC) with di-(2-ethylhexyl) phthalate (DEHP) or ethyl viny acetate (EVA).

- Gently homogenize the diluted solution by inverting the bag. Do not shake.

\subsection{Administration}

- Administer the infusion solution by intravenous infusion using an intravenous tubing infusion set (in PE, PVC with or with a 0.22 micron in-line filter (polyethersulfone [PES], polysulfone, or nylon).

- The infusion solution should be administered for a period of time that will depend on the infusion rate (see Table 2). Use prepared SARCLISA infusion solution within 48 hours when stored refrigerated at $2^{\circ} \mathrm{C}-8^{\circ} \mathrm{C}$, followed by 8 hours

(including the infusion time) at room temperature.

- Do not administer SARCLISA infusion solution concomitantly in the same intravenous line with other agents.

Infusion Rates

Following dilution, administer the SARCLISA infusion

solution intravenously at the infusion rates presented in

Table 2. Incremental escalation of the infusion rate should be considered only in the absence of infusion-related reactions [se Warnings and Precautions (5.1) and Adverse Reactions (6.1)]

Table 2: Infusion Rates of SARCLISA Administration

\begin{tabular}{|l|c|c|c|c|c|}
\hline & $\begin{array}{c}\text { Dilution } \\
\text { Volume }\end{array}$ & $\begin{array}{c}\text { Initial } \\
\text { Rate }\end{array}$ & $\begin{array}{c}\text { Absence of } \\
\text { Infusion- } \\
\text { Related } \\
\text { Reaction }\end{array}$ & $\begin{array}{c}\text { Rate } \\
\text { Increment }\end{array}$ & $\begin{array}{c}\text { Maximum } \\
\text { Rate }\end{array}$ \\
\hline $\begin{array}{l}\text { First } \\
\text { infusion }\end{array}$ & $250 \mathrm{~mL}$ & $\begin{array}{c}25 \mathrm{~mL} / \\
\text { hour }\end{array}$ & $\begin{array}{c}\text { For } 60 \\
\text { minutes }\end{array}$ & $\begin{array}{c}25 \mathrm{~mL} / \text { hour } \\
\text { every } 30 \\
\text { minutes }\end{array}$ & $\begin{array}{c}150 \mathrm{~mL} / \\
\text { hour }\end{array}$ \\
\hline $\begin{array}{l}\text { Second } \\
\text { infusion }\end{array}$ & $250 \mathrm{~mL}$ & $\begin{array}{c}50 \mathrm{~mL} / \\
\text { hour } \\
\text { hour for } 30 \\
\text { minutes then } \\
\text { increase by } \\
100 \mathrm{~mL} / \\
\text { hour every } 30 \\
\text { minutes }\end{array}$ & $\begin{array}{c}\text { For } 30 \\
\text { minutes }\end{array}$ & $\begin{array}{c}200 \mathrm{~mL} / \\
\text { hour }\end{array}$ \\
\hline $\begin{array}{l}\text { Subsequent } \\
\text { infusions }\end{array}$ & $250 \mathrm{~mL}$ & $\begin{array}{c}200 \\
\mathrm{~mL} / \\
\text { hour }\end{array}$ & - & - & $200 \mathrm{~mL} /$ \\
hour
\end{tabular}

\section{CONTRAINDICATIONS}

SARCLISA is contraindicated in patients with severe

hypersensitivity to isatuximab-irfc or to any of its excipients [see Warnings and Precautions (5.1)].

\section{WARNINGS AND PRECAUTIONS}

\subsection{Infusion-Related Reactions}

Infusion-related reactions have been observed in 39\% of

patients treated with SARCLISA [see Adverse Reactions

(6.1)]. All infusion-related reactions started during the first

SARCLISA infusion and resolved on the same day in $98 \%$ of the cases. The most common symptoms of an infusion-

related reaction included dyspnea, cough, chills, and nausea. The most common severe signs and symptoms included hypertension and dyspnea [see Adverse Reactions (6.1)] To decrease the risk and severity of infusion-related reactions, premedicate patients prior to SARCLISA infusion with acetaminophen, $\mathrm{H} 2$ antagonists, diphenhydramine, or cquivalent: dexamethasone [see Dosage and Administration (2.2)]. Monitor vital signs frequently during the entire SARCLISA infusion. For patients with grade 1 or 2 reactions, interrupt SARCLISA infusion and provide appropriate medic support. If symptoms improve, restart SARCLISA infusion at half of the initial infusion rate, with supportive care as needed and closely monitor patients. If symptoms do not recur after 30 minutes, the infusion rate may be increased to the initia rate, and then increased incrementally, as shown in Table 2 [see Dosage and Administration (2.5)]. In case symptoms do not improve or recur after interruption, permanently discontinue SARCLISA and institute appropriate management. Permanently discontinue SARCLISA therapy if a grade 3 or higher infusion-related reaction occurs and institute appropriate medical management.

\subsection{Neutropenia}

SARCLISA may cause neutropenia. Neutropenia (reported as laboratory abnormality) occurred in $96 \%$ of patients and grade 3-4 neutropenia occurred in $85 \%$ of patients treated with SARCLISA, pomalidomide, and dexamethasone (Isa-Pd). Febrile neutropenia occurred in 12\% of patients and neutropenic infections, defined as infection with concurrent grade $\geq 3$ neutropenia, occurred in $25 \%$ of patients treated with Isa-Pd. The most frequent neutropenic infections included those of upper respiratory tract (10\%), lower respiratory tract (9\%), and urinary tract (3\%) [see Adverse Reactions (6.1)].

Monitor complete blood cell counts periodically during treatment. Consider the use of antibiotics and antiviral prophylaxis during treatment. Monitor patients with neutropenia for signs of infection. In case of grade 4 neutropenia delay SARCLISA dose until neutrophil count recovery to at least $1.0 \times 10^{9} / \mathrm{L}$, and provide supportive care with growth factors, according to institutional guidelines. No dose reductions of SARCLISA are recommended.

\subsection{Second Primary Malignancies}

\section{Second primary malignancies were reported in $3.9 \%$ of}

patients in the SARCLISA, pomalidomide and dexamethason (Isa-Pd) arm and in $0.7 \%$ of patients in the pomalidomide an dexamethasone (Pd) arm, and consisted of skin squamous cell carcinoma ( $2.6 \%$ of patients in the Isa-Pd arm and in $0.7 \%$ of patients in the Pd arm), breast angiosarcoma $(0.7 \%$
( $0.7 \%$ of patients in the Isa-Pd arm). With the exception of the patient with myelodysplastic syndrome, patients were able to continue SARCLISA treatment. Monitor patients for the development of second primary malignancies, as per International Myeloma Working Group (IMWG) guidelines. 5.4 Laboratory Test Interference nterference with Serological Testing (Indirect Antiglobulin est) SARCLISA binds to CD38 on red blood cells (RBCs) and may result in a false positive indirect antiglobulin test indirect Coombs test). In ICARIA-multiple myeloma (MM) the indirect antiglobulin test was positive during SARCLISA treatment in $67.7 \%$ of the tested patients. In patients with a positive indirect antiglobulin test, blood transfusions were administered without evidence of hemolysis. ABO/RhD typing administered withoul evidence of hemolysis. ABO/RhD typing SARCLISA infusion, conduct blood type and screen tests on SARCLISA-treated patients. Consider phenotyping prior to starting SARCLISA treatment. If treatment with SARCLISA has already started, inform the blood bank that the patient is receiving SARCLISA and SARCLISA interference with blood compatibility testing can be resolved using dithiothreitoltreated RBCs. If an emergency transfusion is required, noncross-matched ABO/RhD-compatible RBCs can be given as per local blood bank practices [see Drug Interactions (7.1)]. Interference with Serum Protein Electrophoresis and

SARCLISA is an IgG

SARCLISA is an IgG kappa monoclonal antibody that can be incidentally detected on both serum protein electrophoresis and immunofixation assays used for the clinical monitoring of endogenous M-protein. This interference can impact the accuracy of the determination of complete response in some patients with IgG kappa myeloma protein [see Drug Interactions (7.1)].

\subsection{Embryo-Fetal Toxicity}

Based on the mechanism of action, SARCLISA can cause fetal harm when administered to a pregnant woman. SARCLISA may cause fetal immune cell depletion and decreased bon density. Advise pregnant women of the potential risk to a fetus. Advise females with reproductive potential to use an effective method of contraception during treatment with SARCLISA and for at least 5 months after the last dose [see Use in Specific Populations (8.1.8.3)]. The combination of SARCLISA with pomalidomide is contraindicated in pregnant women because pomalidomide may cause birth defects and death of the unborn child. Refer to the pomalidomide prescribing information on use during pregnancy.

\section{ADVERSE REACTIONS}

The following clinically significant adverse reactions from SARCLISA are also described in other sections of the labeling:

- Infusion-Related Reactions [see Warnings and Precautions

(5.1)]

- Neutropenia [see Warnings and Precautions (5.2)]

- Second Primary Malignancies [see Warnings and

Precautions (5.3)]

\subsection{Clinical Trials Experience}

Because clinical trials are conducted under widely varying conditions adverse reaction rates observed in the clinical trials of a drug cannot be directly compared to rates in the clinical trials of another drug and may not reflect the rates observed in practice.

Multiple Myeloma

The safety of SARCLISA was evaluated in ICARIA-MM, a randomized, open-label clinical trial in patients with previously treated multiple myeloma. Patients were eligible for inclusion if they had ECOG status of $0-2$, platelets $\geq 75,000$ cells $/ \mathrm{mm}^{3}$, absolute neutrophil count $\geq 1 \times 10^{9} / \mathrm{L}$, creatinine clearance $\geq 30 \mathrm{~mL} / \mathrm{min}$ (MDRD formula), and AST and/ or ALT $\leq 3 \times$ ULN. Patients received SARCLISA $10 \mathrm{mg} / \mathrm{kg}$ intravenously, weekly in the first cycle and every two weeks thereafter, in combination with pomalidomide and low dose dexamethasone (Isa-Pd) $(n=152)$ or pomalidomide and low dose dexamethasone (Pd) (n=149) [see Clinical Studies (14) in the full prescribing information]. Among patients receiving Isa-Pd, 66\% were exposed to SARCLISA for 6 months or longer and $24 \%$ were exposed for greater than 12 months or longer. The median age of patients who received Isa-Pd was 68 years (range 36-83); $58 \%$ male, $76 \%$ white, and $14 \%$ Asian. Serious adverse reactions occurred in $62 \%$ of patients receiving Isa-Pd. Serious adverse reactions in $>5 \%$ of patients who received Isa-Pd included pneumonia (26\%), upper respiratory tract infections (7\%), and febrile neutropenia $(7 \%)$. Fatal adverse reactions occurred in $11 \%$ of patients (those that occurred in more than $1 \%$ of patients were pneumonia and other infections [3\%]).

Permanent discontinuation due to an adverse reaction (grades 1-4) occurred in 7\% of patients who received Isa-Pd. The most frequent adverse reactions requiring permanent discontinuation in patients who received Isa-Pd were infections $(2.6 \%)$. In addition, SARCLISA alone was discontinued in 3\% of patients due to infusion-related reactions.

Dosage interruptions due to an adverse reaction occurred in $31 \%$ of patients who received SARCLISA. The most frequent adverse reaction requiring dosage interruption was infusionrelated reaction (28\%). 
The most common adverse reactions ( $\geq 20 \%$ ) were neutropenia, infusion-related reactions, pneumonia, upper respiratory tract infection, and diarrhea.

Table 3 summarizes the adverse reactions in ICARIA-MM

Table 3: Adverse Reactions ( $\geq 10 \%)$ in Patients

Receiving SARCLISA, Pomalidomide, and Dexamethasone with a Difference Between Arms of $\geq 5 \%$ Compared to Control Arm in ICARIA-MIM Trial

\begin{tabular}{|l|c|c|c|c|c|c|}
\hline $\begin{array}{l}\text { Adverse } \\
\text { Reactions }\end{array}$ & \multicolumn{2}{|c|}{$\begin{array}{c}\text { SARCLISA+ } \\
\text { Pomalidomide+ } \\
\text { Dexamethasone (Isa-Pd) } \\
\text { (N=152) }\end{array}$} & \multicolumn{3}{|c|}{$\begin{array}{c}\text { Pomalidomide + } \\
\text { Dexamethasone (Pd) } \\
\text { (N=149) }\end{array}$} \\
\hline & $\begin{array}{c}\text { All } \\
\text { grades } \\
(\%)\end{array}$ & $\begin{array}{c}\text { Grade } \\
\mathbf{3} \\
(\%)\end{array}$ & $\begin{array}{c}\text { Grade } \\
\mathbf{4} \\
(\%)\end{array}$ & $\begin{array}{c}\text { All } \\
\text { grades } \\
(\%)\end{array}$ & $\begin{array}{c}\text { Grade } \\
\mathbf{3} \\
(\%)\end{array}$ & $\begin{array}{c}\text { Grade } \\
\mathbf{4} \\
(\%)\end{array}$ \\
\hline $\begin{array}{l}\text { Infusion-related } \\
\text { reaction }\end{array}$ & 38 & 1.3 & 1.3 & 0 & 0 & 0 \\
\hline \begin{tabular}{l} 
Infections \\
\hline Pneumonia*
\end{tabular} & 31 & 22 & 3.3 & 23 & 16 & 2.7 \\
\hline $\begin{array}{l}\text { Upper } \\
\text { respiratorytract } \\
\text { infection }\end{array}$ & 57 & 9 & 0 & 42 & 3.4 & 0 \\
\hline
\end{tabular}

Blood and lymphatic system disorders

\begin{tabular}{|l|c|c|c|c|c|c|}
\hline $\begin{array}{l}\text { Febrile } \\
\text { neutropenia }\end{array}$ & 12 & 11 & 1.3 & 2 & 1.3 & 0.7 \\
\hline
\end{tabular}

Respiratory, thoracic and mediastinal disorders

\begin{tabular}{|l|l|l|l|l|l|l|}
\hline Dyspnea $^{\ddagger}$ & 17 & 5.0 & 0 & 12 & 1.3 & 0 \\
\hline
\end{tabular}

\begin{tabular}{|l|c|c|c|c|c|c|}
\hline \multicolumn{7}{|l|}{ Gastrointestinal disorders } \\
\hline Diarnea & 26 & 2 & - & 19 & 0.7 & - \\
\hline Nausea & 15 & 0 & - & 9 & 0 & - \\
\hline Vomiting & 12 & 1.3 & - & 3.4 & 0 & - \\
\hline
\end{tabular}

CTCAE version 4.03

*Pneumonia includes atypical pneumonia, bronchopulmonary aspergillosis, pneumonia, pneumonia haemophilus, pneumonia influenzal, pneumonia pneumococcal, pneumonia streptococcal, pneumonia viral, candida pneumonia, pneumonia bacterial, haemophilus infection, lung infection, pneumonia fungal, and pneumocystis jirovecii pneumonia.

†Upper respiratory tract infection includes bronchiolitis, bronchitis, bronchitis viral, chronic sinusitis,

fungal pharyngitis, influenza-like illness, laryngitis, nasopharyngitis, parainfluenzae virus infection, pharyngitis, respiratory tract infection, respiratory tract infection viral, rhinitis, sinusitis, tracheitis, upper respiratory tract infection, and upper respiratory tract infection bacterial.

‡Dyspnea includes dyspnea, dyspnea exertional, and dyspnea at rest.

Table 4 summarizes the hematology laboratory abnormalities in ICARIA-MM.

Table 4: Treatment Emergent Hematology Laboratory Abnormalities in Patients Receiving Isa-Pd Treatment versus Pd Treatment - ICARIA-MM

\begin{tabular}{|l|c|c|c|c|c|c|}
\hline $\begin{array}{l}\text { Laboratory } \\
\text { Parameter } \\
\text { n (\%) }\end{array}$ & \multicolumn{3}{|c|}{$\begin{array}{c}\text { SARCLISA+ } \\
\text { Pomalidomide + } \\
\text { Dexamethasone (Isa-Pd) } \\
\text { (N=152) }\end{array}$} & \multicolumn{3}{|c|}{$\begin{array}{c}\text { Pomalidomide + } \\
\text { Dexamethasone (Pd) } \\
\text { (N=149) }\end{array}$} \\
\hline & $\begin{array}{c}\text { All } \\
\text { Grades }\end{array}$ & $\begin{array}{c}\text { Grade } \\
\mathbf{3}\end{array}$ & $\begin{array}{c}\text { Grade } \\
\mathbf{4}\end{array}$ & $\begin{array}{c}\text { All } \\
\text { Grades }\end{array}$ & $\begin{array}{c}\text { Grade } \\
\mathbf{3}\end{array}$ & $\begin{array}{c}\text { Grade } \\
\mathbf{4}\end{array}$ \\
\hline Anemia & 151 & 48 & 0 & $\begin{array}{c}145 \\
(97)\end{array}$ & $\begin{array}{c}41 \\
(28)\end{array}$ & 0 \\
\hline Neutropenia & 146 & $\begin{array}{c}37 \\
(32)\end{array}$ & $\begin{array}{c}92 \\
(96)\end{array}$ & $\begin{array}{c}137 \\
(24)\end{array}$ & $\begin{array}{c}57 \\
(61)\end{array}$ & $\begin{array}{c}46 \\
(31)\end{array}$ \\
\hline Lymphopenia & 140 & $\begin{array}{c}64 \\
(92)\end{array}$ & $\begin{array}{c}19 \\
(13)\end{array}$ & $\begin{array}{c}137 \\
(92)\end{array}$ & $\begin{array}{c}52 \\
(35)\end{array}$ & $\begin{array}{c}12 \\
(8)\end{array}$ \\
\hline Thrombocytopenia & $\begin{array}{c}127 \\
(84)\end{array}$ & $\begin{array}{c}22 \\
(14)\end{array}$ & $\begin{array}{c}25 \\
(16)\end{array}$ & $\begin{array}{c}118 \\
(79)\end{array}$ & $\begin{array}{c}14 \\
(9)\end{array}$ & $\begin{array}{c}22 \\
(15)\end{array}$ \\
\hline
\end{tabular}

\section{Description of Selected Adverse Reactions}

Infusion-related reactions

In ICARIA-MM, infusion-related reactions (defined as adverse reactions associated with the SARCLISA infusions, with an onset typically within 24 hours from the start of the infusion) were reported in 58 patients (38\%) treated with SARCLISA.

All patients who experienced infusion-related reactions, experienced them during the 1st infusion of SARCLISA, with 3 patients (2\%) also having infusion-related reactions at their 2nd infusion, and 2 patients (1.3\%) at their 4th infusion. Grade 1 infusion-related reactions were reported in 3.9\%,

Grade 2 in $32 \%$, Grade 3 in 1.3\%, and Grade 4 in 1.3\% of the patients. Signs and symptoms of Grade 3 or higher infusion-related reactions included dyspnea, hypertension, and bronchospasm. The incidence of infusion interruptions because of infusion-related reactions was $29.6 \%$. The median time to infusion interruption was 55 minutes.

In a separate study (TCD 14079 Part B) with SARCLISA 10 $\mathrm{mg} / \mathrm{kg}$ administered from a $250 \mathrm{~mL}$ fixed-volume infusion in combination with $\mathrm{Pd}$, infusion-related reactions (all Grade 2 were reported in $40 \%$ of patients, at the first administration, the day of the infusion. Overall, the infusion-related reactions of SARCLISA $10 \mathrm{mg} / \mathrm{kg}$ administered as a $250 \mathrm{~mL}$ fixed-volume infusion were similar to that of SARCLISA as administered in ICARIA-MM

Infections

In ICARIA-MM, the incidence of Grade 3 or higher infections was $43 \%$ in Isa-Pd group. Pneumonia was the most commonly reported severe infection with Grade 3 reported in $22 \%$ of patients in Isa-Pd group compared to $16 \%$ in $\mathrm{Pd}$ group, and Grade 4 in $3.3 \%$ of patients in Isa-Pd group compared to $2.7 \%$ in $\mathrm{Pd}$ group. Discontinuations from

reatment due to infection were reported in $2.6 \%$ of patients in Isa-Pd group compared to $5.4 \%$ in $\mathrm{Pd}$ group. Fatal infections were reported in $3.3 \%$ of patients in Isa-Pd group and in $4 \%$ in $\mathrm{Pd}$ group.

\section{Immunogenicity}

As with all therapeutic proteins, there is a potential for immunogenicity. The detection of antibody formation is highly dependent on the sensitivity and specificity of the assay. Additionally, the observed incidence of antibody (including neutralizing antibody) positivity in an assay may be influenced by several factors, including assay methodology, sample handling, timing of sample collection, concomitant medications, and underlying disease. For these reasons, comparison of the incidence of antibodies in the studies described below with the incidence of antibodies in other studies or to other isatuximab-irfc products may be misleading.

In ICARIA-MM, no patients tested positive for antidrug antibodies (ADA). Therefore, the neutralizing ADA status was not determined. Overall, across 6 clinical studies in multiple myeloma (MM) with SARCLISA single agent and combination therapies including ICARIA-MM (N=564), the incidence of treatment emergent ADAs was $2.3 \%$. No clinically significant differences in the pharmacokinetics, safety, or efficacy of isatuximab-irfc were observed in patients with ADAs.

\section{DRUG INTERACTIONS}

\section{Laboratory Test Interference}

Interference with Serological Testing

SARCLISA, an anti-CD38 antibody, may interfere with blood bank serologic tests with false positive reactions in indirect antiglobulin tests (indirect Coombs tests), antibody detection (screening) tests, antibody identification panels, and antihuman globulin crossmatches in patients treated with SARCLISA [see Warnings and Precautions (5.4)]. Interference with Serum Protein Electrophoresis and

Immunofixation Tests

SARCLISA may be incidentally detected by serum protein electrophoresis and immunofixation assays used for the monitoring of M-protein and may interfere with accurate response classification based on International Myeloma Working Group (IMWG) criteria [see Warnings and Precautions (5.4)].

\section{USE IN SPECIFIC POPULATIONS}

\subsection{Pregnancy}

Risk Summary

SARCLISA can cause fetal harm when administered to a pregnant woman. The assessment of isatuximab-irfcassociated risks is based on the mechanism of action and data from target antigen CD38 knockout animal models (see Data). There are no available data on SARCLISA use in pregnant women to evaluate for a drug-associated risk of major birth defects, miscarriage or adverse maternal or fetal outcomes. Animal reproduction toxicity studies have not been conducted with isatuximab-irfc. The estimated background risk of major birth defects and miscarriage for the indicated population is unknown. All pregnancies have a background risk of birth defect, miscarriage, or other adverse outcomes. In the U.S. general population, the estimated background risk of major birth defects and miscarriage in clinically recognized pregnancies is $2 \%$ to $4 \%$ and $15 \%$ to $20 \%$, respectively. The combination of SARCLISA and pomalidomide is contraindicated in pregnant women because pomalidomide may cause birth defects and death of the unborn child. Refer to the pomalidomide prescribing information on use during pregnancy. Pomalidomide is only available through a REMS program

\section{Clinical Considerations}

Fetal/neonatal reactions

Immunoglobulin G1 monoclonal antibodies are known to cross the placenta. Based on its mechanism of action SARCLISA may cause depletion of fetal CD38-positive immune cells and decreased bone density. Defer administration of live vaccines to neonates and infants exposed to SARCLISA in utero until a hematology evaluation is completed. (isatuximab-irfc) injection, for intravenous use

Data

Animal data

Mice that were genetically modified to eliminate all CD38 expression (CD38 knockout mice) had reduced bone density which recovered 5 months after birth. Data from studies using CD38 knockout animal models also suggest the involvement of CD38 in regulating humoral immune responses (mice) feto-maternal immune tolerance (mice), and early embryonic development (frogs)

\subsection{Lactation}

\section{Risk Summary}

There are no available data on the presence of isatuximab-irfo in human milk, milk production, or the effects on the breastfed child. Maternal immunoglobulin $\mathrm{G}$ is known to be present in human milk. The effects of local gastrointestinal exposure and limited systemic exposure in the breastfed infant to SARCLISA are unknown. Because of the potential for serious adverse reactions in the breastfed child from isatuximab-irfc administered in combination with pomalidomide and dexamethasone, advise lactating women not to breastfeed during treatment with SARCLISA. Refer to pomalidomide prescribing information for additional information.

\subsection{Females and Males of Reproductive Potential} Pregnancy Testing

With the combination of SARCLISA with pomalidomide, refer to the pomalidomide labeling for pregnancy testing requirements prior to initiating treatment in females of reproductive potential.

\section{Contraception}

\section{Cemales}

SARCLISA can cause fetal harm when administered to a pregnant woman [see Use in Specific Populations (8.1)]. Advise female patients of reproductive potential to use effective contraception during treatment and for at least 5 months after the last dose of SARCLISA. Additionally, refer to the pomalidomide labeling for contraception requirements prior to initiating treatment in females of reproductive potential

Males

Refer to the pomalidomide prescribing information.

\subsection{Pediatric Use}

Safety and effectiveness in pediatric patients have not been established.

\subsection{Geriatric Use}

Of the total number of subjects in clinical studies of

SARCLISA, 53\% (306 patients) were 65 and over, while 14\% (82 patients) were 75 and over. No overall differences in safety or effectiveness were observed between subjects 65 and over and younger subjects, and other reported clinical experience has not identified differences in responses between the adults 65 years and over and younger patients, but greater sensitivity of some older individuals cannot be ruled out.

\section{OVERDOSAGE}

There is no known specific antidote for SARCLISA overdose. In the event of overdose of SARCLISA, monitor the patients for signs or symptoms of adverse effects and take all

appropriate measures immediately.

Manufactured by:

sanofi-aventis U.S. LLC

Bridgewater, NJ 08807

A SANOFI COMPANY

U.S. License No. 1752

SARCLISA is a registered trademark of Sanof

(C)2020 sanofi-aventis U.S. LLC

ISA-BPLR-SA-MAR20

Revised: March 2020 

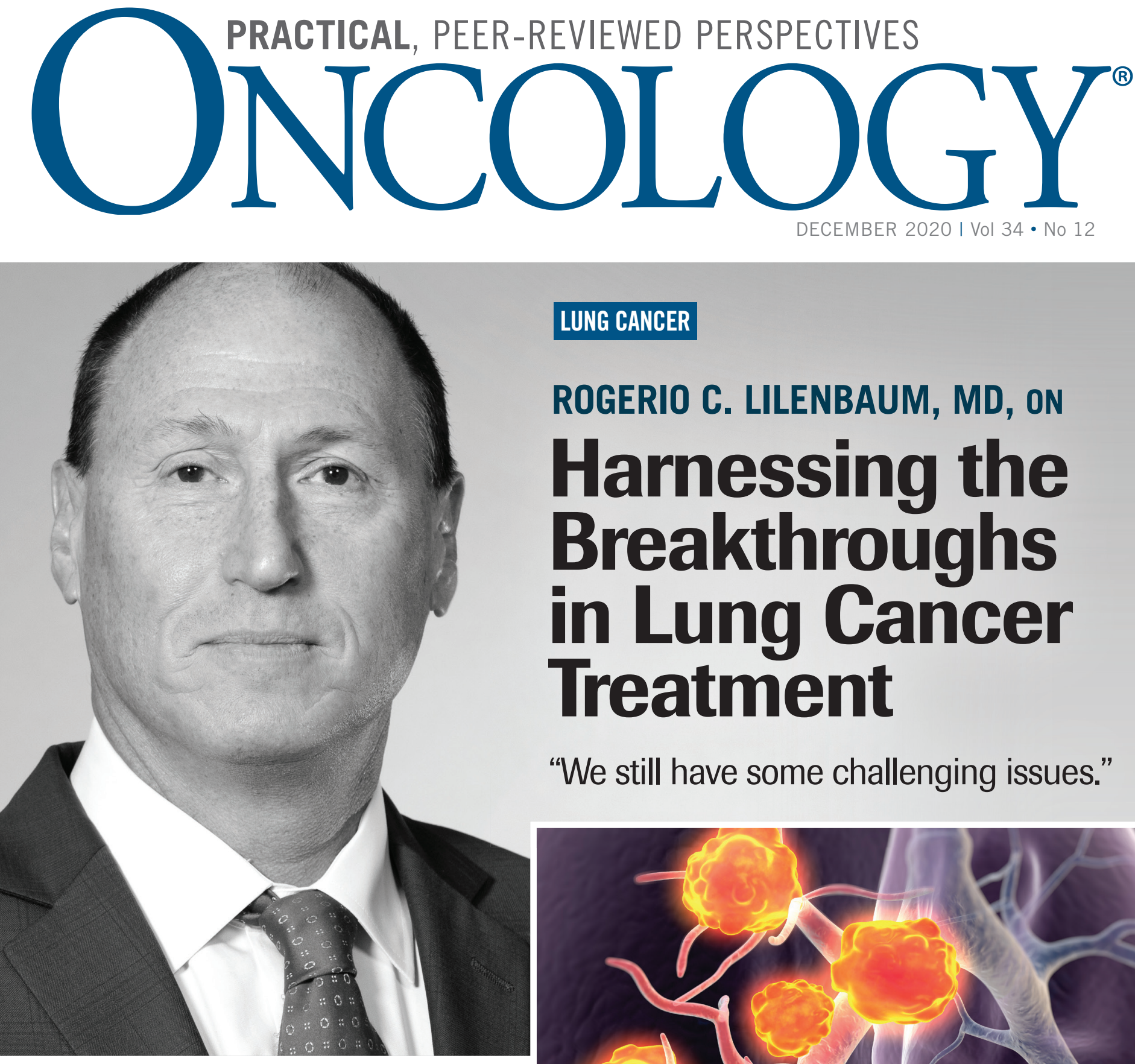

GU Cancer Pipeline

Women's Cancer FDA Approval

GI Cancer Pancreatic Neuroendocrine Tumor With Humoral Hypercalcemia and High

LUNG CANCER

ROGERIO C. LILENBAUM, MD, ON Harnessing the Breakthroughs in Lung Cancer Treatment

"We still have some challenging issues."

Tumor PD-L1 Score Mehmet Sitki Copur, MD, FACP; Luciano Vargas, MD; Whitney Wedel, MD; Shaheed Merani, MD; Allison CushmanVokoun, MD, PhD; Angela Drincic, MD

Immunotherapy Beyond PD-1 Blockade: Novel Checkpoint Inhibitors Naiyer A. Rizvi, MD

Hematological Malignancies Recent Advances in Antibody-Drug Conjugates for Lymphoma David A. Russler-Germain, MD, PhD; Brad S. Kahl, MD

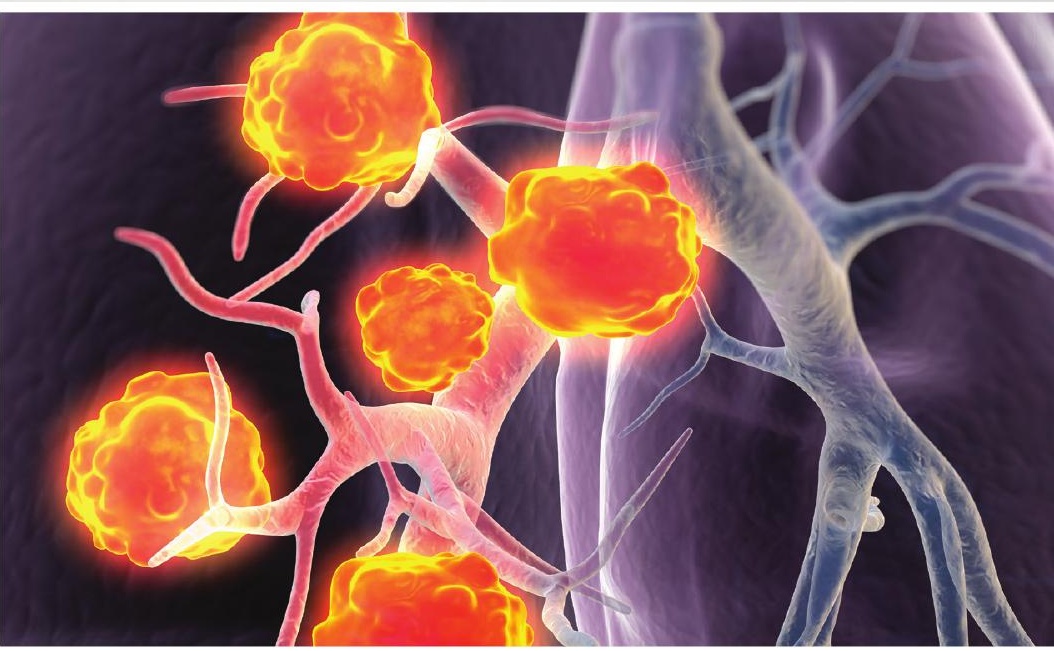




\section{When the path forward in MCRPC is unclear, the Oncotype DX AR-VF Nucleus Detect ${ }^{\circledR}$ test can help navigate.}

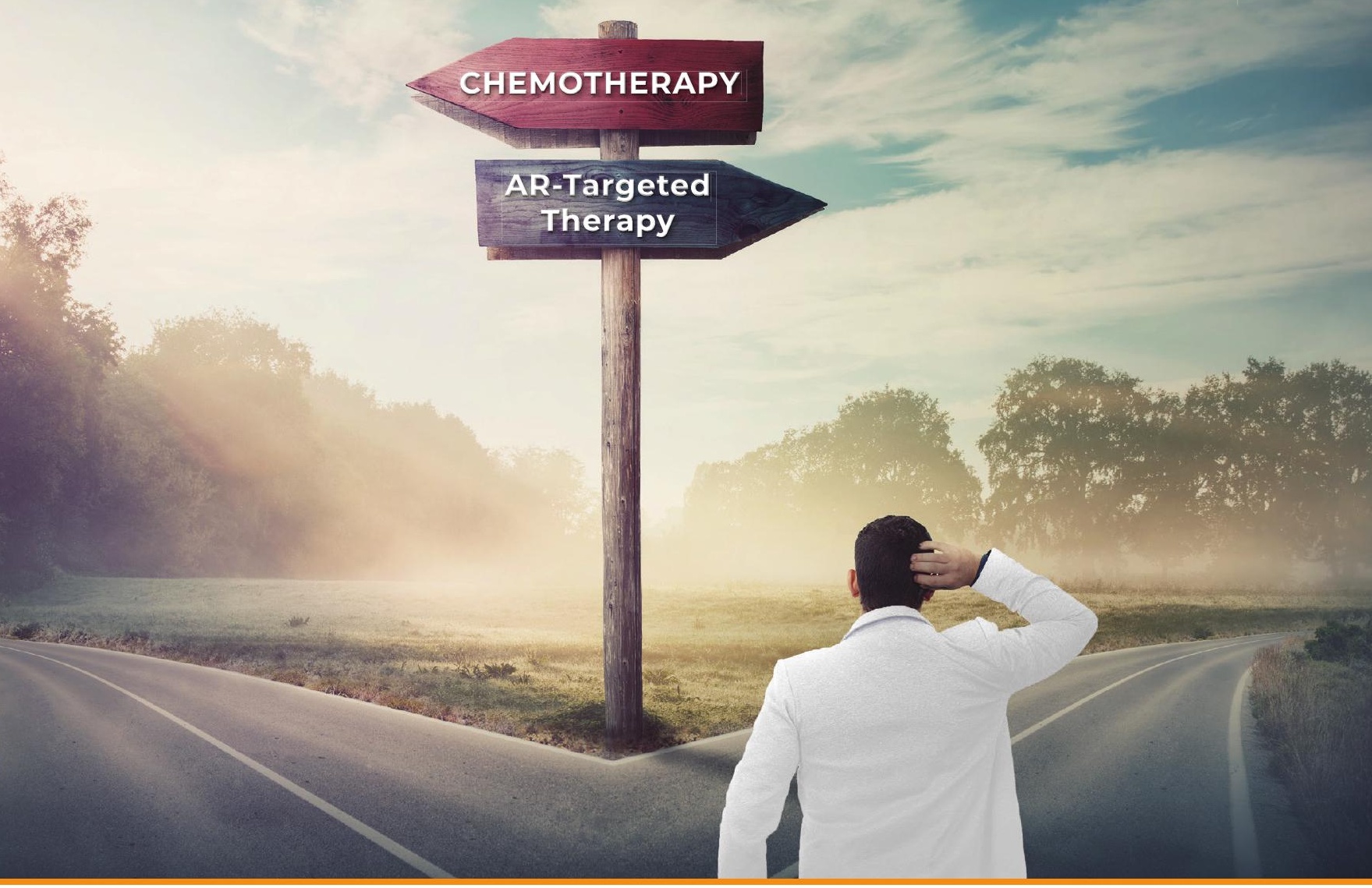

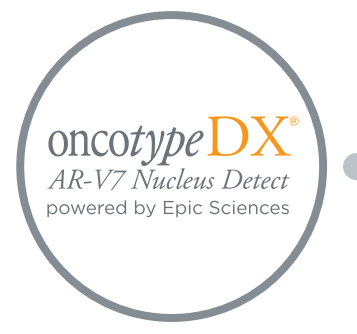

Order the test after a patient fails an AR-targeted therapy

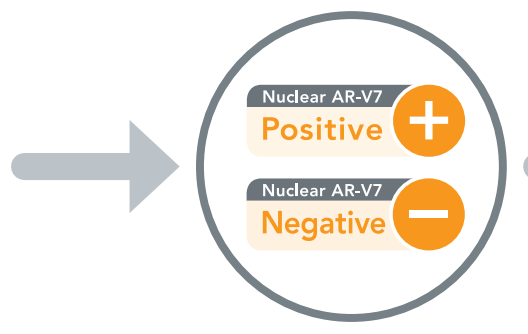

Understand patient's AR-V7 status

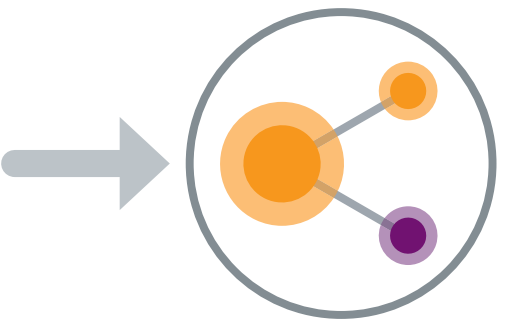

Consider sequential AR-targeted therapy or chemotherapy depending on AR-V7 status 
IN THIS ISSUE

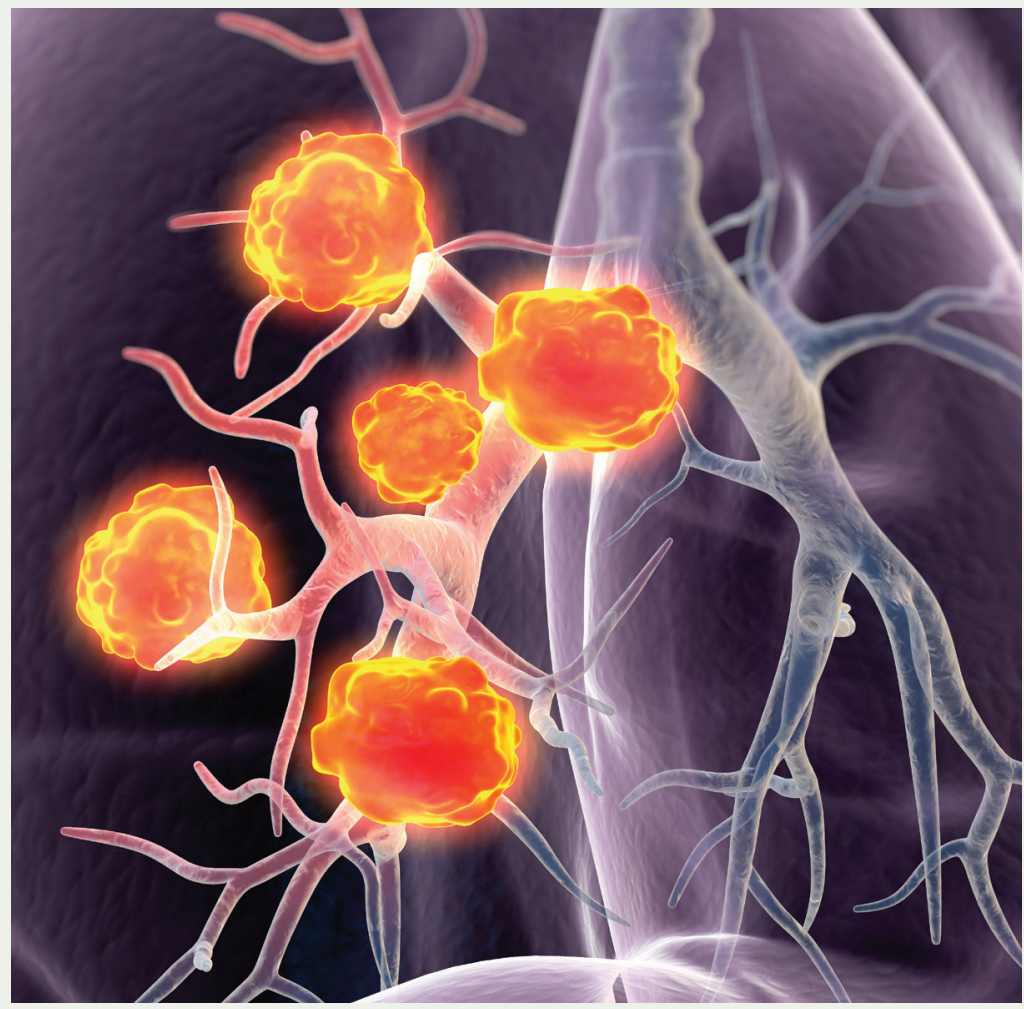

\section{LUNG CANCER: Cover \\ Harnessing the Breakthroughs in Lung Cancer Treatment}

ONCOLOG $Y^{\circledR}$ recently sat down with Rogerio C. Lilenbaum, $\mathrm{MD}$, director of Banner MD Anderson Cancer Center, to discuss current and future trends in the care of patients with lung cancer, as well as his transition from clinician to administrator and the considerable set of challenges that role brings.

\section{Chairman's Letter \\ Optimizing Therapies for Patients With Lung Cancer}

Mike Hennessy Sr

\section{Letter to Readers \\ Molecular Therapy: Lung Cancer Leading the Way}

Howard S. Hochster, MD cancernetwork.com

Visit CancerNetwork.com, home of the journal ONCOLOGY ${ }^{B}$ and a web destination for oncologists seeking expert peer perspectives, podcasts, and other clinically practical features.

\section{NEWS}

Delays in Cancer Treatment Are Linked With Increased Mortality Rates

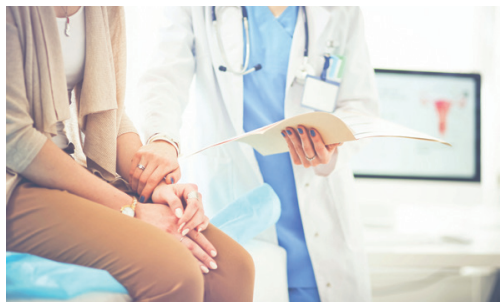

Cancernetwork.com/cancertreatment delay

\section{PODCAST}

\section{Barriers to Pancreatic Cancer Clinical Trial Enrollment}

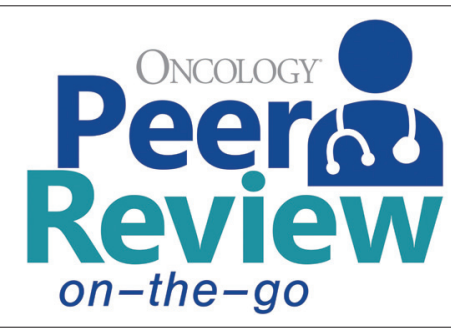

Cancernetwork.com/clinicaltrial_podcast

\section{VIDEO}

Adil Daud, MD, on the Importance of Testing Patients With Melanoma For BRAF Mutations

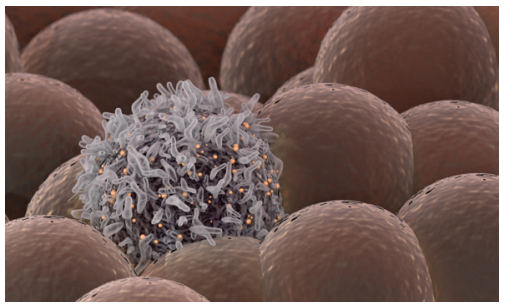

Cancernetwork.com/Daud_BRAF 
IN THIS ISSUE

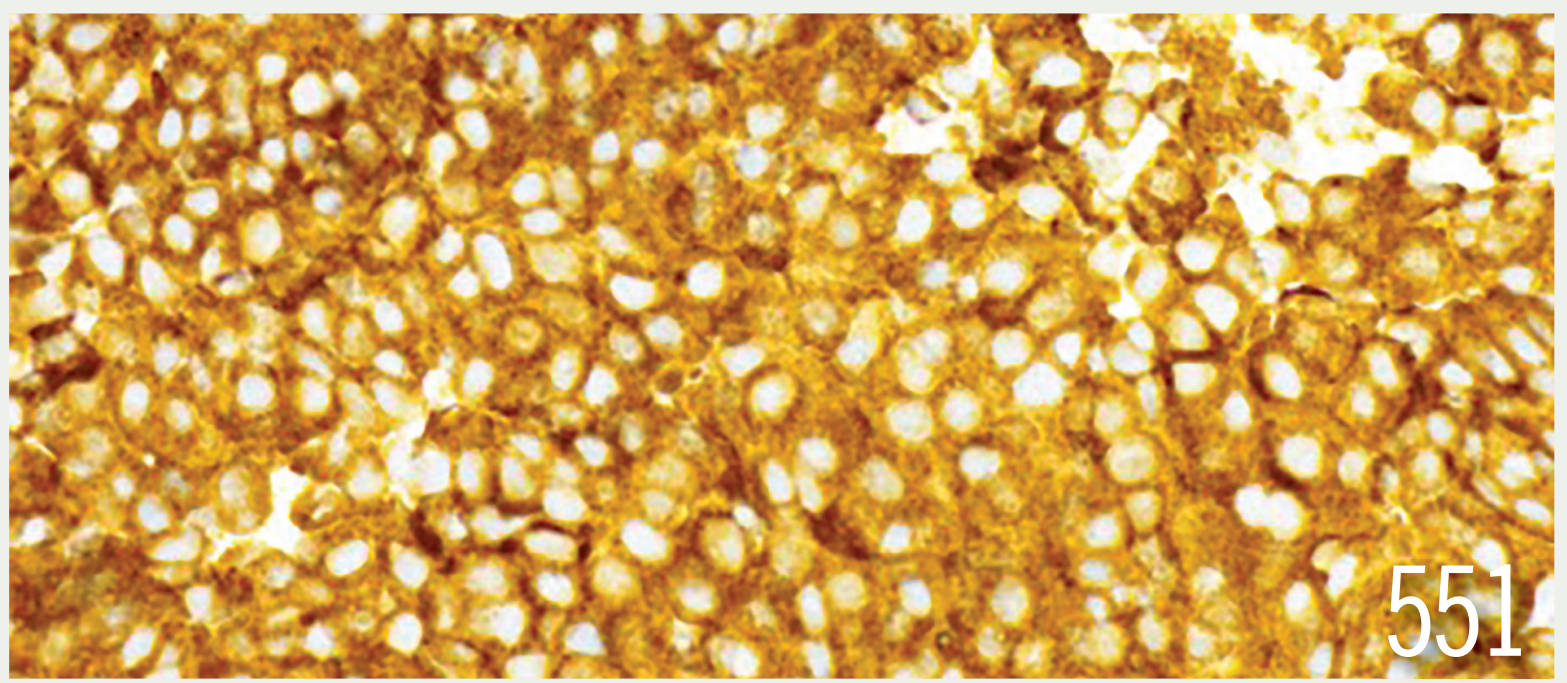

\section{HEMATOLOGICAL \\ MALIGNANCIES: Review \\ Article \\ 522 Recent Advances \\ in Antibody-Drug \\ Conjugates for \\ Lymphoma}

David A. Russler-Germain, MD, PhD;

Brad S. Kahl, MD

\section{GU CANCER: Pipeline}

539 Updates on Key

Clinical Trials and Drug

Development

Published in affiliation with

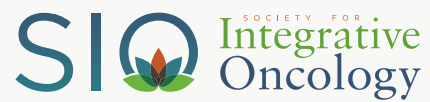

WOMEN'S CANCER: FDA

Approval

547 FDA Approves

Pembrolizumab

+ Chemotherapy

Combination for Locally

Recurrent Unresectable or Metastatic TNBC

Hannah Slater

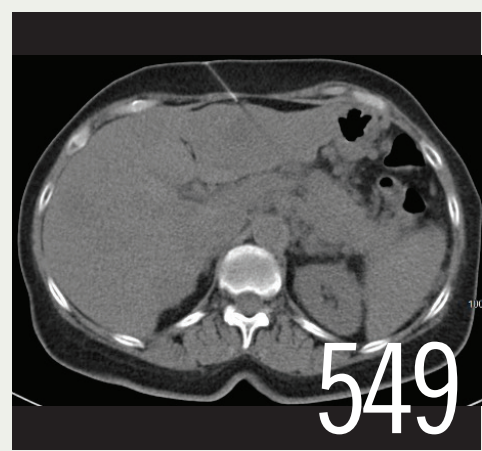

GI CANCER: Case Study 548 Pancreatic Neuroendocrine Tumor With Humoral Hypercalcemia and High Tumor PD-L1 Score

Mehmet Sitki Copur, MD, FACP; Luciano Vargas, MD; Whitney Wedel, MD; Shaheed Merani, MD; Allison Cushman-Vokoun, MD, PhD; Angela Drincic, MD

IMMUNOTHERAPY: Continuing Medical Education 553 Beyond PD-1 Blockade: Novel Checkpoint Inhibitors

Naiyer A. Rizvi, MD

JPR THE editors ARE PLEASED to ANNOUNCE the availability of our new parent company's continuing education activities. gotoper.com We've picked this one especially for our ONCOLOGY ${ }^{\circledR}$ readers. Go to: https://bit.ly/2IRAknZ

Advertiser and advertising agency recognize and accept that the following language appears within the publication: "All statements, including product claims, are those of the person or organization making the statement or claim. The publisher does not adopt any such statement or claim as its own, and any such statement or claim does not necessarily reflect the opinion of the publisher." (including text, representations, illustrations, opinions and facts) of advertisements printed, and also assume responsibility for any claims made against the publisher arising from or related to such advertisements. In the event that legal action or a claim is made against the publisher arising from or related to such advertisements, advertiser and advertising agency agree to fully defend, indemnify and hold harmless the publisher, and to pay any judgment, expenses and legal fees incurred by the publisher as a result of said legal action or claim. The publisher eserves the right to reject any advertising which he feels is not in keeping with the publication's standards.

Publisher is not liable fordelays in delivery and/ornon-deliveninthe event of an act of God, action by any govermment or quasi-governmental entity, fire, flood, insurrection, riot, explosion, embargo, strikes (whether legal or illegal), labor or materia shortage, transportation interruption of any kind, work slow-down, or any condition beyond the control of publisher affecting production or delivery in any manner.
ONCOLOG\% (ISSN 0890-9091) is published monthly by MultiMedia Healthcare LLC, 2 Clarke Drive, Suite 100 Cranbury, N 08512. Annual subscription rates: US, \$199 and Canada, \$219; students and nurses, \$96; international, \$249. Single copies: $\$ 20$ each. Institutional US, $\$ 299$; Canada, \$329; international, \$375. Periodicals postage paid at Trenton, $\mathrm{NJ}$ and at additional mailing offices. POSTMASTER: Please send address changes to Oncology PO Box 457, Cranbury NJ 08512-0457, USA. Publications Mail Agreement № 40612608. Return Undeliverable Canadian Addresses to: IMEX Global Solutions, P0 Box 25542 London ON N6C 6B2. Canadian G.S.T number: R-124213133RT001. Printed in U.S.A.

For address changes, please notify the Circulation Department by visiting www.surveymonkey.com/s/subscriptions, or by mail to ONCOLOGY , ( 2020 MH Life Sciences, PO Box 457, Cranbury NJ 08512-0457. Send old address, new address and attach a copy of mail label, if possible. 


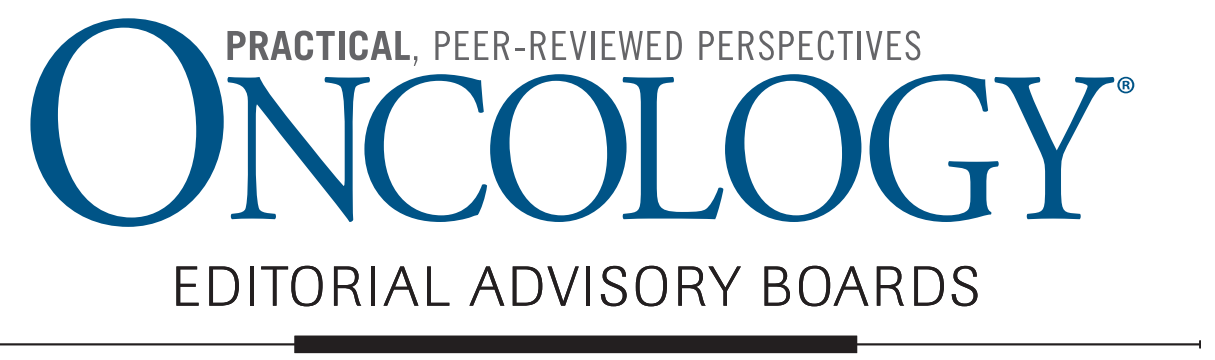

MISSION STATEMENT

ONCOLOGY $Y^{\circledR}$ and its website, CancerNetwork.com, provide oncologists with the practical, timely, clinical information they need to deliver the highest level of care to their patients. Expert authors and peer review ensure the quality of ONCOLOGY ${ }^{\circledR}$ and CancerNetwork.com's articles and features. Focused discussions capture key clinical take-aways for application in today's time-constrained practice environment.

\section{EDITORS-IN-CHIEF}

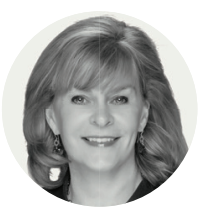

Julie M. Vose, MD, MBA Omaha, NE
GASTROINTESTINAL CANCER

Tanios S. Bekaii-Saab, MD, Phoenix, AZ

HEAD AND NECK CANCER

Eric J. Sherman, MD, New York, NY
HEMATOLOGIC MALIGNANCIES

C. Ola Landgren, MD, PhD, New York, NY

LUNG CANCER

Joshua M. Bauml, MD, Philadelphia, PA

\section{BOARD MEMBERS}

BREAST CANCER

William J. Gradishar, MD, FACP, Chicago, IL

Tari King, MD, Boston, MA

Vered Stearns, MD, Baltimore, MD

Melinda L. Telli, MD, Palo Alto, CA

CANCER SURVIVORSHIP

Matthew J. Matasar, MD, MS, New York, NY

COLORECTALGGSTROINTESTINAL CANCER

Edward Chu, MD, Pittsburgh, PA

Mehmet Sitki Copur, MD, FACP, Omaha, NE

Daniel Haller, MD, Philadelphia, PA

John L. Marshall, MD, Washington, DC

Shubham Pant, MD, Houston, TX

Matthew B. Yurgelun, MD, Boston, MA

Mehmet Sitki Copur, MD, FACP, Omaha, NE

GENITOURINARY CANCER

L. Michael Glodé, MD, FACP, Denver, CO

Paul Mathew, MD, Boston, MA

Elisabeth Heath, MD, FACP, Detroit, MI

William Oh, MD, New York, NY

GYNECOLOGIC ONCOLOGY

Mario M. Leitao Jr, MD, New York, NY

Franco Muggia, MD, New York, NY
HEAD AND NECK CANCER

Apar K. Ganti, MD, MS, FACP, Omaha, NE

HEALTH ECONOMICS

Nora Janjan, MD, Dallas, TX

HEMATOLOGIC MALIGNANCIES

Danielle M. Brander, MD, Durham, NC

Christopher R. Flowers, MD, Houston, TX

Steven T. Rosen, MD, Duarte, CA

Naval G. Daver, MD, Houston, TX

Ehab L. Atallah, MD, Milwaukee, WI

INFECTIOUS DISEASE

Genovefa Papanicolaou, MD, New York, NY

INTEGRATIVE ONCOLOGY

Ting Bao, MD, New York, NY

Linda Carlson, PhD, RPsych, Calgary, Alberta,

Canada

LUNG CANCER

David S. Ettinger, MD, Baltimore, MD James L. Mulshine, MD, Chicago, IL

Edward S. Kim, MD, Charlotte, NC

Jennifer W. Carlisle, MD, Atlanta, GA

MELANOMA

Richard D. Carvajal, MD, New York, NY

Jason Luke, MD, FACP, Pittsburgh, PA

\section{NEURO-ONCOLOGY}

David A. Reardon, MD, Boston, MA Stuart A. Grossman, MD, Baltimore, MD Nicole A. Shonka, MD, Omaha, NE

PEDIATRIC ONCOLOGY

David G. Poplack, MD, Houston, TX Richard A. Drachtman, MD, New Brunswick, NJ

PROSTATE CANCER

Tomasz M. Beer, MD, Portland, OR

E. David Crawford, MD, Denver, CO

Judd W. Moul, MD, FACS, Durham, NC

PSYCHO-ONCOLOGY

Daniel C. McFarland, Do, New York, NY

Michelle Riba, MD, Ann Arbor, MI

RADIATION ONCOLOGY

Louis Potters, MD, FACR, Hempstead, NY James B. Yu, MD, MHS, New Haven, CT

SARCOMA

Kenneth Cardona, MD, FACS, Atlanta, GA

SUPPORTIVE AND PALLIATIVE CARE

Thomas J. Smith, MD, FACP, Baltimore, MD

N. Simon Tchekmedyian, MD, Long Beach, CA

SURGICAL ONCOLOGY

Burton L. Eisenberg, MD, Newport Beach, CA

INTERESTED IN SUBMITTING TO ONCOLOGY ${ }^{\circledR}$ ?

Please contact senior editor Kevin Wright at KWright@mjhlifesciences.com for submission guidelines or more information. 


\section{Optimizing Therapies For Patients With Lung Cancer}

The incidence and overall mortality of lung cancer has been declining in the United States, due in part to both reduced use of tobacco and better treatment options. Despite those treatment options, for patients with advanced disease, lung cancer remains difficult to treat.

In this issue of ONCOLOGY ${ }^{\circledR}$, we spoke with Rogerio C. Lilenbaum, MD, director of Banner MD Anderson Cancer Center in Gilbert, Arizona, about the recent treatment breakthroughs for patients with lung cancer, and the obstacles that those breakthroughs bring.

"I think we still have some challenging issues," Lilenbaum says. "Testing in this day and age is still erratic," adds Lilenbaum. "Even within each category - EGFR, ALK, ROS, RET, BRAF - the information about how to best manage those patients is not always within the domain of the average oncologist."

Lilenbaum also notes that identifying patients who benefit from immunotherapy and checkpoint inhibitors is also an issue. "Yes, PD-L1 is a marker that can help ascertain that magnitude of benefit, but it's far from perfect," said Lilenbaum. "I think we need additional predictors or predictive factors to justify the cost and the toxicity of these agents."

Also in this issue, you will read a review of the recent advances in antibody-drug conjugates for patients with lymphoma. With the clinical successes of both polatuzumab vedotin (Polivy) and brentuximab vedotin (Adcetris), and several promising agents under development, this unique class of chemoimmunotherapy agents is poised to make a large impact in the treatment of lymphoma.

We also present a case of a patient with a metastatic pancreatic neuroendocrine tumor with hypercalcemia, elevated parathyroid hormone-related peptide, high tumor mutational burden, and high PD-L1 score. How do we treat a patient who presents with this rare condition? Read on to find out.

Within these pages, you'll also read about some of the emerging therapeutics and diagnostic devices in the genitourinary cancer pipeline and a discussion of the FDA's recent accelerated approval of pembrolizumab (Keytruda) in combination with chemotherapy for the treatment of patients with locally recurrent unresectable or metastatic triple-negative breast cancer.

I hope you find our journal helpful in caring for your patients through what is likely one of the most challenging times in their lives. As always, thank you for reading.

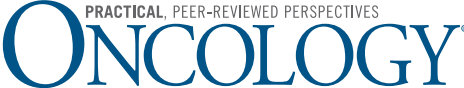

DECEMBER 2020 - VOL. 34 • NO. 12

\section{EDITORIAL}

KRISTIE L. KAHL Editorial Director

KEVIN WRIGHT Senior Editor

HANNAH SLATER Assistant Editor

MATTHEW FOWLER Assistant Editor

JENNIFER POTASH Copy Chief

RACHELLE LALIBERTE, PAUL SILVERMAN Copy Supervisors

KELLY KING Senior Copy Editor

CHENEY BALTZ, GEORGINA CARSON, REBEKAH HARRISON, KIRSTY MACKAY, RON PANAROTTI

Copy Editors

\section{DESIGN \& PRODUCTION}

ROBERT MCGARR Creative Director

KRISTEN MORABITO Art Director

RACHEL KEATLEY Graphic Designer

JONATHAN SEVERN Circulation Director

KEYONNA GRAHAM Production Director

\section{PUBLISHING \& SALES}

BRIAN HAUG Executive Vice President 609-325-4780 • bhaug@mmhgroup.com

MICHELLE JANIN Director of Sales

732-429-4316•mjanin@mmhgroup.com

PATRIC PASTORE National Accounts Associate 609-955-1694•ppastore@mjhlifesciences.com

\section{AUDIENCE DEVELOPMENT}

KELLY KEMPER Audience Development Manager

\section{CORPORATE}

MIKE HENNESSY SR Chairman \& Founder

JACK LEPPING Vice Chairman

MIKE HENNESSY JR President \& CEO

NEIL GLASSER, CPA/CFE Chief Financial Officer

MICHAEL BAER Chief Marketing Officer

JOE PETROZIELLO Executive Vice President, Global Medical Affairs and Corporate Development

SILAS INMAN Senior Vice President, Content

MICHAEL BALL Senior Vice President Operations

JOHN MORICONE Senior Vice President, I.T. \& Enterprise Systems

SHARI LUNDENBERG Vice President. Human Resources \& Administration

CHRIS HENNESSY Vice President, Mergers \& Acquisitions JEFF BROWN Executive Creative Director, Creative Services

\section{SUBSCRIPTIONS}

888-527-7008 


\title{
LETTER TO THE READERS
}

\section{Molecular Therapy: Lung Cancer Leads the Way}

\author{
Howard S. Hochster, MD \\ Distinguished Professor of Medicine, Rutgers Robert Wood Johnson Medical School \\ Associate Cancer Center Director, Rutgers Cancer Institute of New Jersey
}

$\mathrm{T}$ his month, ONCOLOGY ${ }^{\circledR}$ features an interview with Rogerio C. Lilenbaum, MD, who outlines the evolution in treatment of non-small cell lung cancer (NSCLC), from the era of platinum doublets to highly targeted therapies. Lilenbaum is now director of the Banner MD Anderson Cancer Center in Arizona, and his interview brings to mind memories of when we worked together.

Approximately 8 years ago, I was fortunate to be a faculty member at Yale Cancer Center along with 3 world-class thoracic oncologists, Thomas J. Lynch Jr, MD; Roy S. Herbst, MD, PhD; and Lilenbaum. I have to admit that I was extremely jealous of the treatment landscape for NSCLC.

Lynch, the cancer center's director at the time, was responsible for the observation that gefitinib (Iressa), a new drug targeting EGFR, was highly active in a patient who had an EGFR mutation. This conclusion was based on the spectacular clinical response of a young woman who was a nonsmoker, and recent work related to gene sequencing that was being performed at the Broad Institute. The initial report of the efficacy of gefitinib in patients harboring NSCLC tumors with activating EGFR mutations was published in the New England Journal of Medicine. ${ }^{1}$ This was a great revelation, because gefitinib was not active for $90 \%$ of the patients with NSCLC being treated. Oncology as a discipline has never looked back from this paradigm of driver mutations being treated with tyrosine kinase inhibitors.

About the same time, Herbst came to Yale following a successful tenure at MD Anderson, where he had instituted the BATTLE trial to evaluate multiple treatments for NSCLC in the metastatic setting. ${ }^{2}$ This was further developed to look at targeted agents and evolved into the SWOG LUNGMAP (NCT03851445) trial, which continues to enroll and develop agents in new strategies for lung cancer.

Recently, we have seen progress with the "undruggable" target of RAS. This gene is a GTPase rather than having adenosine triphosphate as a substrate (which is the case for all the other targeted drugs, going back to imatinib). This unscalable mountain is being whittled away with drugs targeting specific mutations, locking them in the off position. The latest drugs from Amgen (AMG 510) and Mirati (MRTX849) attack the GTPase of the G12C mutation by interacting with the cysteine atom. These have shown a $30 \%$ response rate in NSCLC but substantially lower in colon cancer with the same mutation. How this target will contribute to eventual therapy remains to be seen, but they may be the first cracks in the RAS wall of resistance. The promise of effective RAS drugs in pancreatic cancer, which has an $85 \%$ mutation rate, is hard to overestimate.

At the time these observations were moving forward in NSCLC, the field of colon cancer was quite far behind in the availability of targeted therapies. Developing a multiarm drug trial was just not possible because of limited numbers of patients with treatable, actionable mutations. Today, we are somewhat more fortunate with understanding of microsatellite unstable tumors, poor-prognosis $B R A F$-mutated colon cancer and effective triple-drug therapy, HER 2 amplification and antibody therapy, and the rare NTRK fusions that are treatable now with targeted agents. Remarkably, we still can prognosticate more effectively on the basis of right-versus-left-sided colon cancer than on molecular profile.

The 2020 holidays are approaching, and it is sometimes hard to grasp that for which we should be thankful, given the disruption to our lives in this year of the coronavirus disease 2019 pandemic. However, I am thankful for the observations of those in lung cancer therapy who made the key observations and developed the important paradigms of the new oncology: next-generation sequencing, understanding driver mutations, and development of inhibitors. We have come a long way from empiric broad-based chemotherapy drugs to treating tumors with the appropriate targeted agents. I consider the term precision medicine somewhat of an optimistic overstatement, but at least it is a state we can aspire to and work toward.

For full reference list, visit: cancernetwork.com/Dec_LTR 


\title{
Recent Advances in Antibody-Drug Conjugates for Lymphoma
}

David A. Russler-Germain, MD, PhD'; and Brad S. Kahl, MD'

'Division of Oncology, Department of Medicine, Siteman Cancer Center, Washington University School of Medicine, St Louis, MO

\begin{abstract}
Antibody-drug conjugates (ADCs) comprise a unique class of chemoimmunotherapy agents, incorporating cytotoxic payloads covalently linked to a monoclonal antibody via specialized linkers. This strategy attempts to exploit antibody-antigen specificity to selectively deliver a potent 'warhead' payload to tumor cells (Figure), while sparing nontumor antigen-negative cells. Decades of development have culminated in the recent approvals of a handful of ADCs across multiple tumor types. ADCs for the treatment of lymphoma are particularly attractive due in part to the favorable spectrum of cell surface markers uniquely expressed on lymphocytes compared with other tissues. Here we discuss general principles of ADC design, including antigen/ antibody, payload, and linker selection. We highlight the clinical successes of the 2 approved ADCs for treatment of lymphomas: brentuximab vedotin (Adcetris) and polatuzumab vedotin (Polivy). Finally, we describe several $A D C$ agents currently under development for lymphoma, including emerging efficacy and toxicity data from early-stage clinical trials (Table).
\end{abstract}

\section{Introduction}

The mainstay of treatment for the majority of lymphomas has been systemic cytotoxic chemotherapy, either single-agent or multiagent, for many decades. Advances in biotechnology through the 1990s facilitated the development of the anti-CD20 monoclonal antibody ( $\mathrm{mAb}$ ) rituximab (Rituxan), which is now widely used both as a monotherapy and in combination with chemotherapy for the treatment of a wide range of $\mathrm{B}$-cell malignancies (in addition to being used for several nonmalignant conditions). ${ }^{1}$ Oral and in- travenous (IV) targeted small molecule therapies have demonstrated impressive efficacy in various frontline and relapsed settings for a range of lymphoid malignancies, ${ }^{2}$ but balancing the effectiveness and toxicities of lymphoma treatments remains a key goal in this area. The lymphoma community has continued to seek novel therapies that specifically target tumor cells, as the lineage-specific agent rituximab does, which would allow for increasingly potent treatments to be offered to patients by selectively delivering cytotoxic agents to sites of disease while sparing healthy tissues from drug exposure. Antibody-drug conjugates (ADCs) are one manifestation of this "magic bullet" that was historically imagined by Paul Ehrlich. ${ }^{3}$ Two ADCs have been approved for the treatment of lymphomas; several other agents are being actively explored and showing early clinical promise. Complex factors must be considered when constructing potential ADCs, as these multicomponent cancer therapies come with compounding levels of nuance in their design strategies.

\section{Antigen Selection and Antibody Design}

The fundamental goal of an ADC is the tumor-specific delivery of a cytotoxic drug, so it is vital to identify an appropriate antigenic target for an ADC's mAb component. This first major hurdle has been overcome with varying degrees of ease or difficulty depending on tumor type. For epithelial malignancies, which can share considerable cell surface marker expression with other organs of endodermal origin, the identification of tumor-specific instead of tissue-specific antigens has been one key to successful ADC development. ${ }^{4}$ In contrast, multiple lymphoid lineage-specific markers are ripe for exploitation by ADCs for the treatment of lymphomas, as any nontumor B- and/or T-cell ablation can 
often be tolerated, and minimal nonhematopoietic end-organ toxicities would be predicted. Later in this review, we outline many of the cell surface markers currently being utilized for lymphoma ADC development, including their spectrum of expression in different hematopoietic compartments plus other tissues. Not only does one strive for high tumor cell expression of an ADC target antigen compared with limited or no expression in other tissues, but this target antigen must also localize to the cell surface to be accessible to the circulating ADC. Upon binding, the ADC should then be transported intracellularly for release of its cytotoxic payload. Some cell surface antigens, however, are noninternalizing, creating additional challenges for drug development.

Due to the unique mechanism of action of ADCs, it is not required for their mAb component to possess any intrinsic non-ADC ("naked") clinical activity. Furthermore, many ADCs are also the first agents to show clinical activity targeting a particular antigen. The anti-CD30 ADC brentuximab vedotin (Adcetris; BV), as described in more detail below, is an example of both principles, and there are also similar examples in diseases other than lymphoma. Additionally, whether the mAb used in an ADC exerts any antibody-dependent cellular cytotoxicity (ADCC) or complement-dependent cytotoxicity (CDC) could complicate the potential adverse event (AE) profile of the ADC, separate from the expected toxicities that may be caused by off-target exposure to the actual cytotoxic payload of the ADC.

Lastly, while not specific to ADCs, $\mathrm{mAb}$ design in general must also address immunogenicity and isotype selection. Whether a $\mathrm{mAb}$ is chimeric, humanized, or fully human will influence the potential for antidrug antibodies (ADAs) to emerge. This phenomenon has been shown to be detrimental to ADC effectiveness in a variety of settings. ${ }^{5}$ Most ADCs utilize immunoglobulin (Ig) G

\section{FIGURE. General Principles of ADCs.}

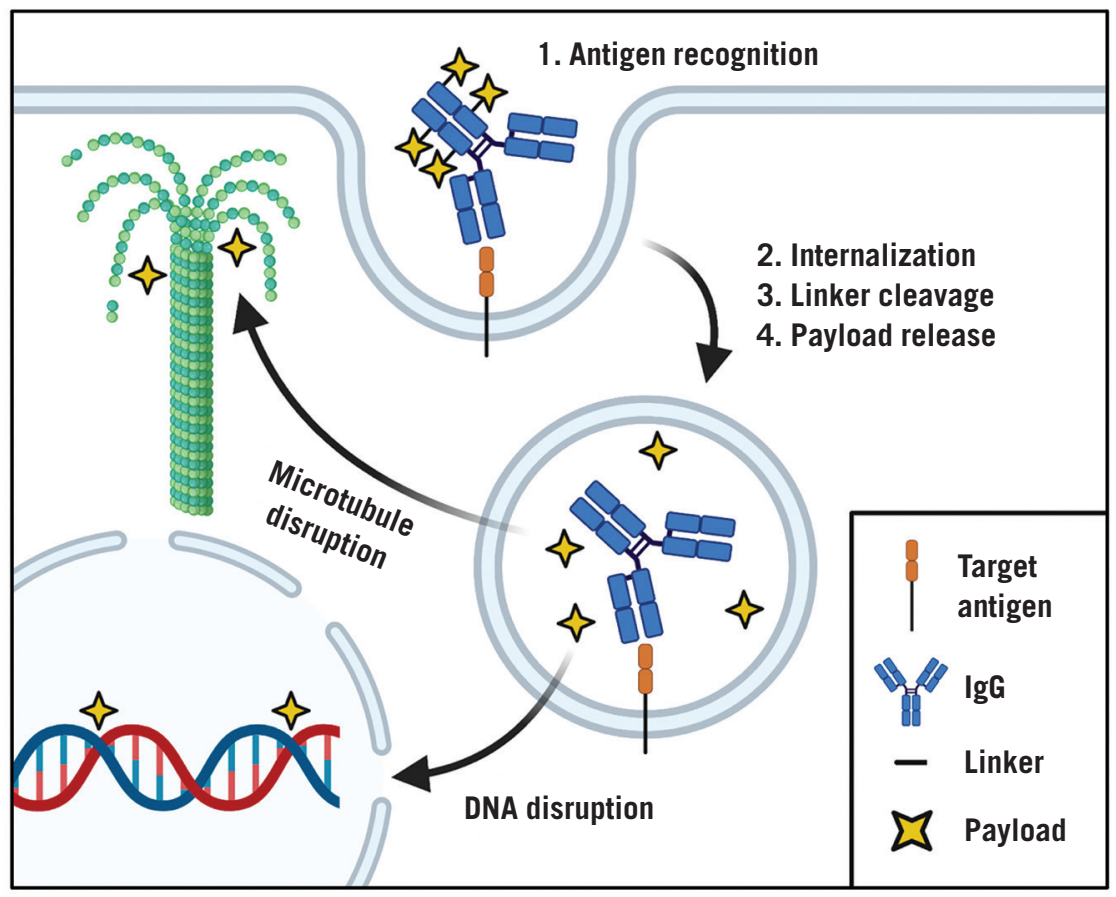

IgG, immunoglobulin G.

Illustration created with BioRender.com.

mAbs, and the choice of specific isotype impacts production ease, payload linkage ease, and pharmacokinetics, as well as potential AEs when the ADC is administered clinically. ${ }^{6-8}$ IgG3 mAbs suffer from faster clearance, making them largely unsuited for use in ADCs. IgG1, IgG2, and IgG4 mAbs have differing numbers of disulfide bonds, which potentially impact where and how many payloads can be affixed. Further, the potential for ADCC and CDC varies among isotypes, with more for IgG1 and less for IgG2 or IgG4. Related to this, recent advances in $\mathrm{mAb}$ design have explored the introduction of mutations to modulate these $\mathrm{mAb}$ functions. One aim has been to abrogate off-target effects via silencing mutations of the Fc domain, thus minimizing FcyR binding and theoretically reducing subsequent thrombocytopenia, which has been observed with the anti-HER2 ADC trastu- zumab emtansine (Kadcyla) for breast cancer. ${ }^{9}$ In other contexts, Fc domain mutations have been introduced to enhance ADCC by increasing the mAb affinity for FcyRIIIa, such as with an anti-BCMA ADC for multiple myeloma. ${ }^{10}$

\section{Choice of Payload}

The ADCs that are currently approved utilize a relatively narrow set of cytotoxic payloads as "warheads" due to a number of limitations, but extensive active research continues in this arena. Most ADCs achieve drug-to-antibody ratios between $3: 1$ and 5:1, and the absolute number of target antigens expressed on the tumor cell surface is in the range of $10^{3}$ to $10^{7}$ in most circumstances. ${ }^{11}$ This results in relatively low absolute drug delivery to a tumor cell in terms of intracellular cytotoxic agent concentration compared with the concentrations commonly achieved 
by systemic IV chemotherapy. In turn, most ADCs take advantage of payloads that are highly potent, often with a half maximal inhibitory concentration in the range of $10^{-10}$ to $10^{-12}$ molar. These drugs commonly target microtubules (and so are cytotoxic to proliferating cells) or DNA (and so are cytotoxic to both proliferating and nonproliferating cells). Most frequently used are auristatins, such as monomethyl auristatin $\mathrm{E}$ (MMAE) and F, which are microtubule disrupting agents. ${ }^{12}$ These are synthetic analogues of dolastatin 10, a pentapeptide derived from the marine mollusk Dolabella auricularia but which is too toxic for use outside of the ADC context. Next most common are ADCs with payloads derived from maytansine, a natural product of the African shrub Maytenus ovatus that also disrupts microtubules; examples include drug maytansinoid (DM)1 and DM4. ${ }^{13}$ Also under investigation are tubulysins, which comprise a third, less common class of ADC payloads that targets microtubules.

Of the payloads that target DNA, common classes include calicheamicins, which bind the minor groove of DNA and induce site-specific double-strand cleavage, and duocarmycins, which are alkylating agents. ${ }^{14}$ Also under development are several ADCs with pyrrolobenzodiazepine (PBD) payloads, which are naturally occurring antitumor antibiotics that dimerize then bind and crosslink DNA. ${ }^{15}$ ADCs with camptothecin analogues, such as prodrugs of the established chemotherapeutic agent irinotecan, inhibit DNA topoisomerase I. They are already being advanced in nonlymphoma settings and have the benefits of particularly high potency and water solubility. ${ }^{16}$ Finally, seveal other classes of cytotoxic payloads are in preclinical development, including RNA polymerase II inhibitors, crytophycin-based tubulin inhibitors, novel anthracyclines, and RNA spliceosome inhibitors.

\section{Linker Optimization}

In addition to $\mathrm{mAb}$ and cytotoxic payload selection, optimizing the linkage between these 2 components plays a vital role in the toxicity, efficaciousness, and manufacturing ease of an ADC. On one hand, an ADC must remain stable in circulation and tissues-for a period ranging from days to potentially weeks-without releasing its cytotoxic payload and harming areas without tumor. On the other, once internalized, an ADC must make the payload available to exert its cytotoxic effects, often by conditional release in specific subcellular compartments. Some linkers being explored are noncleavable, which would make ADCs dependent on proteolytic degradation of the $\mathrm{mAb}$ to release its payload. ${ }^{17}$ In contrast, cleavable linkers are also utilized, such as those sensitive to lysosomal proteases, acidic $\mathrm{pH}$, or reduction by glutathione, which all trigger intracellular dissociation of the payload from the mAb. ${ }^{18}$ Despite generally aiming to avoid extracellular release of the toxic payload of ADCs, strategies to overcome heterogeneity of antigen expression in tumors have included tolerating and even intentionally exploiting a "bystander effect." ${ }^{19}$ Released payloads (often with part of the linker attached) that are able to cross cellular membranes have the potential to reach nearby antigen-negative tumor cells, which could enhance an ADC's therapeutic effect. Nonpolar agents have the greatest potential for exhibiting a beneficial bystander effect, but this can come with disadvantages beyond toxic exposure to the payload. Specifically, increasing linker-payload hydrophobicity can induce circulating $\mathrm{ADC}$ aggregation, accelerate $\mathrm{ADC}$ clearance, and lead to reduced ADC potency in tumors expressing MDR1.20,21 In the end, striking the perfect balance between excessive and ineffective local exposure to the toxic payload can be difficult. Overall, there is no single optimal strategy for ADC development as it pertains to these aforementioned issues; instead, there are complex tradeoffs with each decision that impact efficacy and toxicity, often unique to each target antigen and tumor type.

\section{Brentuximab Vedotin}

CD30 is a member of the tumor necrosis factor (TNF) receptor superfamily and is traditionally expressed on the surface of subsets of $\mathrm{T}$ cells, B cells, and macrophages. ${ }^{22}$ However, its characteristic expression in a variety of lymphoid malignancies and its rapid endocytic internalization upon binding by a $\mathrm{mAb}$ made it an attractive target for the development of the ADC BV. BV consists of a chimeric anti-CD30 IgG1 mAb with the antitubulin agent MMAE attached via a protease-sensitive dipeptide valine-citrulline linker. ${ }^{23}$ Pharmacokinetic analyses revealed that steady-state concentrations of BV are achieved with dosing every 3 weeks, such that minimal to no accumulation occurs with repeated dosing. ${ }^{24}$

The multicenter, open-label, phase 1 dose-escalation study of BV explored doses between 0.1 and $3.6 \mathrm{mg} / \mathrm{kg}$ administered every 21 days. ${ }^{25}$ It enrolled 45 patients with relapsed or refractory $(\mathrm{R} / \mathrm{R})$ CD30-positive hematologic malignancies, primarily Hodgkin lymphoma (HL) and anaplastic large cell lymphoma (ALCL). The maximum tolerated dose (MTD) was $1.8 \mathrm{mg} / \mathrm{kg}$ and the most common AEs were fatigue, pyrexia, diarrhea, nausea, neutropenia, and peripheral neuropathy. Initial regulatory approval was derived from a subsequent multicenter, openlabel, phase 2 study that carried forward the MTD of $1.8 \mathrm{mg} / \mathrm{kg}$, treating $102 \mathrm{pa}-$ tients with $\mathrm{HL}$ relapsing after autologous stem cell transplantation (autoSCT). ${ }^{26}$ The ORR was $75 \%$, with complete remission (CR) in $34 \%$ of patients. The median progression-free survival (PFS) for all patients was only 5.6 months, but the median duration of response (DOR) for those achieving $\mathrm{CR}$ was a promising 20.5 months. The confirmatory trial,

Continued on page 530 

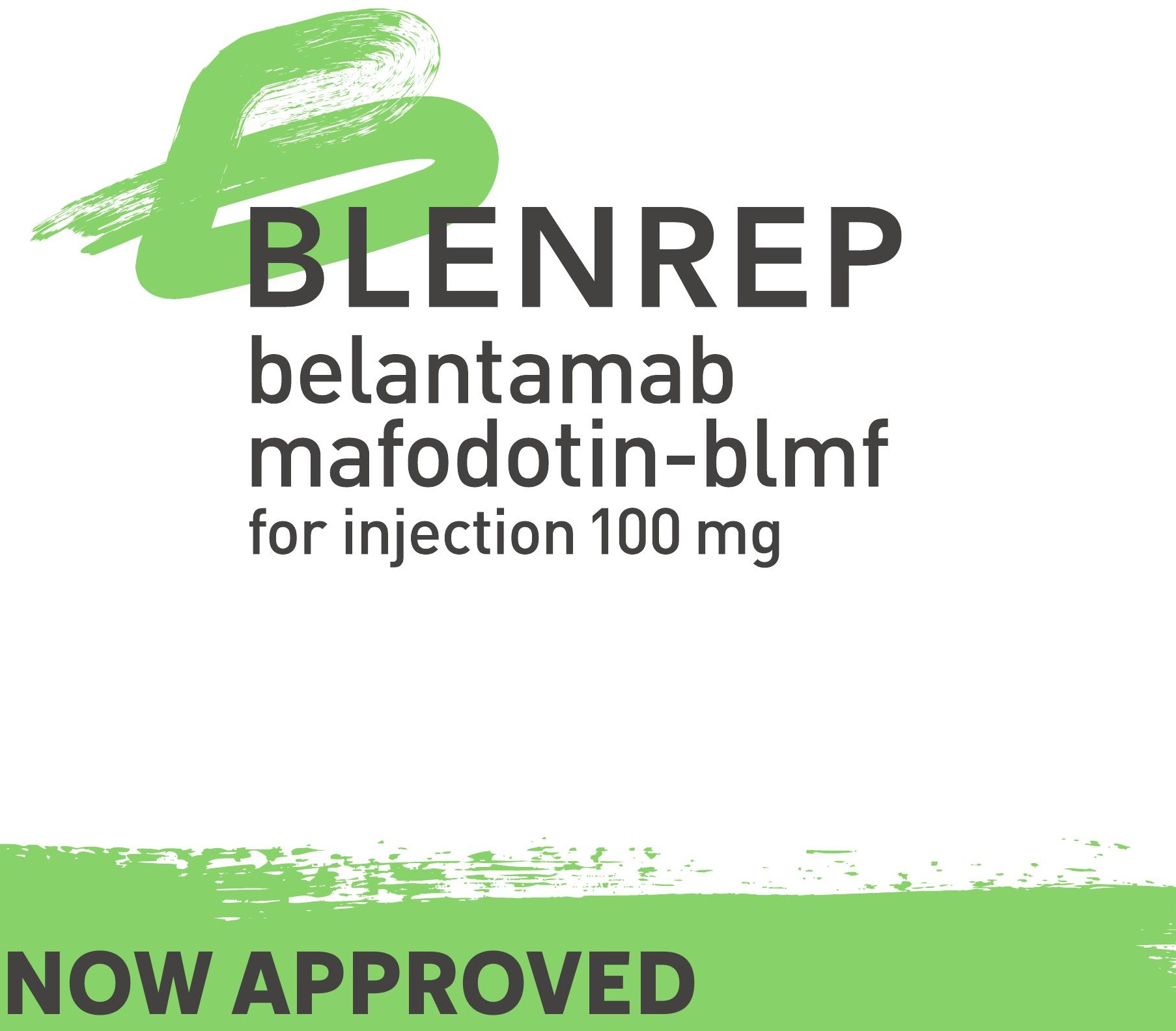

Please see following pages for Brief Summary of full Prescribing Information, including BOXED WARNING

Trademarks are owned by or licensed

to the GSK group of companies. 


\section{BRIEF SUMMARY \\ BLENREP}

\section{(belantamab mafodotin-blmf) for injection, for intravenous use}

The following is a brief summary only; see full Prescribing Information for complete product information.

\section{WARNING: OCULAR TOXICITY}

BLENREP caused changes in the corneal epithelium resulting in changes in vision, including severe vision loss and corneal ulcer, and symptoms, such as blurred vision and dry eyes [see Warnings and Precautions (5.1)]

Conduct ophthalmic exams at baseline, prior to each dose, and promptly for worsening symptoms. Withhold BLENREP until improvement and resume, or permanently discontinue, based on severity [see Dosage and Administration (2.3), Warnings and Precautions (5.1)].

Because of the risk of ocular toxicity, BLENREP is available only through a restricted program under a Risk Evaluation and Mitigation Strategy (REMS) called the BLENREP REMS [see Warnings and Precautions (5.2)].

\section{INDICATIONS AND USAGE}

BLENREP is indicated for the treatment of adults with relapsed or refractory multiple myeloma who have received at least 4 prior therapies, including an anti-CD38 monoclonal antibody, a proteasome inhibitor, and an immunomodulatory agent.

This indication is approved under accelerated approval based on response rate [see Clinical Studies (14) of full Prescribing Information]. Continued approval for this indication may be contingent upon verification and description of clinical benefit in a confirmatory trial(s).

\section{DOSAGE AND ADMINISTRATION}

\subsection{Important Safety Information}

Perform an ophthalmic exam prior to initiation of BLENREP and during treatment [see Warnings and Precautions (5.1)].

Advise patients to use preservative-free lubricant eye drops and avoid contact lenses unless directed by an ophthalmologist [see Warnings and Precautions (5.1)].

\subsection{Recommended Dosage}

The recommended dosage of BLENREP is $2.5 \mathrm{mg} / \mathrm{kg}$ of actual body weight given as an intravenous infusion over approximately 30 minutes once every 3 weeks until disease progression or unacceptable toxicity.

\subsection{Dosage Modifications for Adverse Reactions}

The recommended dose reduction for adverse reactions is:

- BLENREP $1.9 \mathrm{mg} / \mathrm{kg}$ intravenously once every 3 weeks.

Discontinue BLENREP in patients who are unable to tolerate a dose of $1.9 \mathrm{mg} / \mathrm{kg}$ (see Tables 1 and 2).

\section{Corneal Adverse Reactions}

The recommended dosage modifications for corneal adverse reactions, based on both corneal examination findings and changes in best-corrected visual acuity (BCVA), are provided in Table 1 [see Warnings and Precautions (5.1)]. Determine the recommended dosage modification of BLENREP based on the worst finding in the worst affected eye. Worst finding should be based on either a corneal examination finding or a change in visual acuity per the Keratopathy and Visual Acuity (KVA) scale.

Table 1. Dosage Modifications for Corneal Adverse Reactions per the KVA Scale

\begin{tabular}{|l|l|l|}
\hline \multicolumn{2}{|l|}{ Corneal Adverse Reaction } & $\begin{array}{l}\text { Recommended Dosage } \\
\text { Modifications }\end{array}$ \\
\hline Grade 1 & $\begin{array}{l}\text { Corneal examination finding(s): } \\
\text { Mild superficial keratopathy } \\
\text { Change in BCVA } \\
\text { Decline from baseline of 1 line } \\
\text { on Snellen Visual Acuity }\end{array}$ & $\begin{array}{l}\text { Continue treatment at current } \\
\text { dose. }\end{array}$ \\
\hline Grade 2 & $\begin{array}{l}\text { Corneal examination finding(s): } \\
\text { Moderate superficial keratopathyc } \\
\text { Change in BCVA : } \\
\text { Decline from baseline of 2 or 3 } \\
\text { lines on Snellen Visual Acuity and } \\
\text { not worse than 20/200 }\end{array}$ & $\begin{array}{l}\text { Withhold BLENREP until } \\
\text { improvement in both corneal } \\
\text { examination findings and } \\
\text { change in BCVA to Grade 1 } \\
\text { or better and resume at same } \\
\text { dose. }\end{array}$ \\
\hline
\end{tabular}

Table 1. Dosage Modifications for Corneal Adverse Reactions per the KVA Scale (continued)

\begin{tabular}{|c|c|c|}
\hline Grade 3 & $\begin{array}{l}\text { Corneal examination finding(s): } \\
\text { Severe superficial keratopathy } \\
\text { Change in } B C V A^{\mathrm{b}} \text { : } \\
\text { Decline from baseline by more } \\
\text { than } 3 \text { lines on Snellen Visual } \\
\text { Acuity and not worse than } 20 / 200\end{array}$ & $\begin{array}{l}\text { Withhold BLENREP until } \\
\text { improvement in both corneal } \\
\text { examination findings and } \\
\text { change in BCVA to Grade } \\
1 \text { or better and resume at } \\
\text { reduced dose. }\end{array}$ \\
\hline Grade 4 & $\begin{array}{l}\text { Corneal examination finding(s): } \\
\text { Corneal epithelial defect } \mathrm{e}^{\mathrm{s}} \\
\text { Change in } B C V A^{\mathrm{b}} \text { : } \\
\text { Snellen Visual Acuity worse than } \\
20 / 200\end{array}$ & $\begin{array}{l}\text { Consider permanent } \\
\text { discontinuation of BLENREP. } \\
\text { If continuing treatment, } \\
\text { withhold BLENREP until } \\
\text { improvement in both corneal } \\
\text { examination findings and } \\
\text { change in BCVA to Grade } \\
1 \text { or better and resume at } \\
\text { reduced dose. }\end{array}$ \\
\hline
\end{tabular}

a Mild superficial keratopathy (documented worsening from baseline), with or without symptoms.

${ }^{b}$ Changes in visual acuity due to treatment-related corneal findings.

'Moderate superficial keratopathy with or without patchy microcyst-like deposits, sub-epithelial haze (peripheral), or a new peripheral stromal opacity.

${ }^{d}$ Severe superficial keratopathy with or without diffuse microcyst-like deposits, sub-epithelial haze (central), or a new central stromal opacity.

${ }^{e}$ Corneal epithelial defect such as corneal ulcers.

Other Adverse Reactions

The recommended dosage modifications for other adverse reactions are provided in Table 2.

Table 2. Dosage Modifications for Other Adverse Reactions

\begin{tabular}{|l|l|l|}
\hline \multirow{2}{*}{ Adverse Reaction } & Severity & $\begin{array}{l}\text { Recommended Dosage } \\
\text { Modifications }\end{array}$ \\
\hline $\begin{array}{l}\text { Thrombocytopenia } \\
\text { [see Warnings and } \\
\text { Precautions (5.3)] }\end{array}$ & $\begin{array}{l}\text { Platelet count } \\
25,000 \text { to less than } \\
50,000 / \mathrm{mcL}\end{array}$ & $\begin{array}{l}\text { Consider withholding BLENREP and/or } \\
\text { reducing the dose of BLENREP. }\end{array}$ \\
\cline { 2 - 3 } & $\begin{array}{l}\text { Platelet count less } \\
\text { than 25,000/mcL }\end{array}$ & $\begin{array}{l}\text { Withhold BLENREP until platelet } \\
\text { count improves to Grade 3 or better. } \\
\text { Consider resuming at a reduced dose. }\end{array}$ \\
\hline $\begin{array}{l}\text { Infusion-related } \\
\text { reactions } \\
\text { [see Warnings and } \\
\text { Precautions (5.4)] }\end{array}$ & $\begin{array}{l}\text { Grade 2 (moderate) } \\
\text { or Grade 3 (severe) }\end{array}$ & $\begin{array}{l}\text { Interrupt infusion and provide } \\
\text { supportive care. Once symptoms } \\
\text { resolve, resume at lower infusion } \\
\text { rate; reduce the infusion rate by at } \\
\text { least 50\%. }\end{array}$ \\
\cline { 2 - 3 } & $\begin{array}{l}\text { Grade 4 } \\
\text { (life-threatening) }\end{array}$ & $\begin{array}{l}\text { Permanently discontinue BLENREP } \\
\text { and provide emergency care. }\end{array}$ \\
\hline $\begin{array}{l}\text { Other Adverse } \\
\text { Reactions [see } \\
\text { Adverse Reactions } \\
\text { (6.1)] }\end{array}$ & Grade 3 & $\begin{array}{l}\text { Withhold BLENREP until improvement } \\
\text { to Grade 1 or better. Consider } \\
\text { resuming at a reduced dose. }\end{array}$ \\
\cline { 2 - 3 } & Grade 4 & $\begin{array}{l}\text { Consider permanent discontinuation } \\
\text { of BLENREP. If continuing treatment, } \\
\text { withhold BLENREP until improvement } \\
\text { to Grade 1 or better and resume at } \\
\text { reduced dose. }\end{array}$ \\
\hline
\end{tabular}

\subsection{Preparation and Administration}

BLENREP is a hazardous drug. Follow applicable special handling and disposal procedures.

Calculate the dose $(\mathrm{mg})$, total volume $(\mathrm{mL})$ of solution required, and the number of vials of BLENREP needed based on the patient's actual body weight. More than 1 vial may be needed for a full dose. Do not round down for partial vials.

\section{Reconstitution}

- Remove the vial(s) of BLENREP from the refrigerator and allow to stand for approximately 10 minutes to reach room temperature $\left(68^{\circ} \mathrm{F}\right.$ to $77^{\circ} \mathrm{F}\left[20^{\circ} \mathrm{C}\right.$ to $\left.\left.25^{\circ} \mathrm{C}\right]\right)$.

- Reconstitute each 100-mg vial of BLENREP with $2 \mathrm{~mL}$ of Sterile Water for Injection, USP, to obtain a final concentration of $50 \mathrm{mg} / \mathrm{mL}$. Gently swirl the vial to aid dissolution. Do not shake.

- If the reconstituted solution is not used immediately, store refrigerated at $36^{\circ} \mathrm{F}$ to $46^{\circ} \mathrm{F}$ $\left(2^{\circ} \mathrm{C}\right.$ to $8^{\circ} \mathrm{C}$ ) or at room temperature $\left(68^{\circ} \mathrm{F}\right.$ to $77^{\circ} \mathrm{F}\left[20^{\circ} \mathrm{C}\right.$ to $\left.\left.25^{\circ} \mathrm{C}\right]\right)$ for up to 4 hours in the original container. Discard if not diluted within 4 hours. Do not freeze. 
- Parenteral drug products should be inspected visually for particulate matter and discoloration prior to administration, whenever solution and container permit. The reconstituted solution should be clear to opalescent, colorless to yellow to brown liquid. Discard if extraneous particulate matter is observed.

\section{Dilution}

- Withdraw the calculated volume of BLENREP from the appropriate number of vials and dilute in a $250-\mathrm{mL}$ infusion bag of $0.9 \%$ Sodium Chloride Injection, USP, to final concentration of $0.2 \mathrm{mg} / \mathrm{mL}$ to $2 \mathrm{mg} / \mathrm{mL}$. The infusion bags must be made of polyvinylchloride (PVC) or polyolefin (PO).

- Mix the diluted solution by gentle inversion. Do not shake.

- Discard any unused reconstituted solution of BLENREP left in the vial(s).

- If the diluted infusion solution is not used immediately, store refrigerated at $36^{\circ} \mathrm{F}$ to $46^{\circ} \mathrm{F}\left(2^{\circ} \mathrm{C}\right.$ to $\left.8^{\circ} \mathrm{C}\right)$ for up to 24 hours. Do not freeze. Once removed from refrigeration, administer the diluted infusion solution of BLENREP within 6 hours (including infusion time).

- Parenteral drug products should be inspected visually for particulate matter and discoloration prior to administration, whenever solution and container permit. The diluted infusion solution should be clear and colorless. Discard if particulate matte is observed.

\section{Administration}

- If refrigerated, allow the diluted infusion solution to equilibrate to room temperature $\left(68^{\circ} \mathrm{F}\right.$ to $77^{\circ} \mathrm{F}$ [ $20^{\circ} \mathrm{C}$ to $\left.\left.25^{\circ} \mathrm{C}\right]\right)$ prior to administration. Diluted infusion solution may be kept at room temperature for no more than 6 hours (including infusion time).

- Administer by intravenous infusion over approximately 30 minutes using an infusion set made of polyvinyl chloride (PVC) or polyolefin (PO).

- Filtration of the diluted solution is not required; however, if the diluted solution is filtered, use a polyethersulfone (PES)-based filter (0.2 micron).

Do not mix or administer BLENREP as an infusion with other products. The product does not contain a preservative.

\section{CONTRAINDICATIONS}

None.

\section{WARNINGS AND PRECAUTIONS}

\subsection{Ocular Toxicity}

Ocular adverse reactions occurred in $77 \%$ of the 218 patients in the pooled safety population. Ocular adverse reactions included keratopathy $(76 \%)$, changes in visual acuity (55\%), blurred vision (27\%), and dry eye (19\%) [see Adverse Reactions (6.1)]. Among patients with keratopathy $(n=165), 49 \%$ had ocular symptoms, $65 \%$ had clinically relevant visual acuity changes (decline of 2 or more lines on Snellen Visual Acuity in any eye), and 34\% had both ocular symptoms and visual acuity changes.

\section{Keratopathy}

Keratopathy was reported as Grade 1 in $7 \%$ of patients, Grade 2 in $22 \%$, Grade 3 in $45 \%$, and Grade 4 in $0.5 \%$ per the KVA scale. Cases of corneal ulcer (ulcerative and infective keratitis) have been reported. Most keratopathy events developed within the first 2 treatment cycles (cumulative incidence of $65 \%$ by Cycle 2). Of the patients with Grade 2 to 4 keratopathy ( $n=149$ ), $39 \%$ of patients recovered to Grade 1 or lower after median follow-up of 6.2 months. Of the $61 \%$ who had ongoing keratopathy, $28 \%$ were still on treatment, $9 \%$ were in follow-up, and in $24 \%$ the follow-up ended due to death, study withdrawal, or lost to follow up. For patients in whom events resolved, the median time to resolution was 2 months (range: 11 days to 8.3 months).

\section{Visual Acuity Changes}

A clinically significant decrease in visual acuity of worse than $20 / 40$ in the betterseeing eye was observed in $19 \%$ of the 218 patients and of $20 / 200$ or worse in the better-seeing eye in $1.4 \%$. Of the patients with decreased visual acuity of worse than $20 / 40,88 \%$ resolved and the median time to resolution was 22 days (range: 7 days to 4.2 months). Of the patients with decreased visual acuity of $20 / 200$ or worse all resolved and the median duration was 22 days (range: 15 to 22 days).

\section{Monitoring and Patient Instruction}

Conduct ophthalmic examinations (visual acuity and slit lamp) at baseline, prior to each dose, and promptly for worsening symptoms. Perform baseline examinations within 3 weeks prior to the first dose. Perform each follow-up examination at least 1 week after the previous dose and within 2 weeks prior to the next dose. Withhold BLENREP until improvement and resume at same or reduced dose, or consider permanently discontinuing based on severity [see Dosage and Administration (2.3)]. Advise patients to use preservative-free lubricant eye drops at least 4 times a day starting with the first infusion and continuing until end of treatment. Avoid use of contact lenses unless directed by an ophthalmologist [see Dosage and
Administration (2.1)].

Changes in visual acuity may be associated with difficulty for driving and reading. Advise patients to use caution when driving or operating machinery.

BLENREP is only available through a restricted program under a REMS [see Warnings and Precautions (5.2)].

\subsection{BLENREP REMS}

BLENREP is available only through a restricted program under a REMS called the BLENREP REMS because of the risks of ocular toxicity [see Warnings and Precautions (5.1)].

Notable requirements of the BLENREP REMS include the following:

- Prescribers must be certified with the program by enrolling and completing training in the BLENREP REMS.

- Prescribers must counsel patients receiving BLENREP about the risk of ocular toxicity and the need for ophthalmic examinations prior to each dose.

- Patients must be enrolled in the BLENREP REMS and comply with monitoring.

- Healthcare facilities must be certified with the program and verify that patients are authorized to receive BLENREP.

- Wholesalers and distributers must only distribute BLENREP to certified healthcare facilities.

Further information is available, at www.BLENREPREMS.com and 1-855-209-9188.

\subsection{Thrombocytopenia}

Thrombocytopenia occurred in $69 \%$ of 218 patients in the pooled safety population, including Grade 2 in 13\%, Grade 3 in 10\%, and Grade 4 in 17\% [see Adverse Reactions (6.1)]. The median time to onset of the first thrombocytopenic event was 26.5 days. Thrombocytopenia resulted in dose reduction, dose interruption, or discontinuation in $9 \%, 2.8 \%$, and $0.5 \%$ of patients, respectively.

Grade 3 to 4 bleeding events occurred in $6 \%$ of patients, including Grade 4 in 1 patient. Fatal adverse reactions included cerebral hemorrhage in 2 patients.

Perform complete blood cell counts at baseline and during treatment as clinically indicated. Consider withholding and/or reducing the dose based on severity [see Dosage and Administration (2.3)].

\subsection{Infusion-Related Reactions}

Infusion-related reactions occurred in $18 \%$ of 218 patients in the pooled safety population, including Grade 3 in 1.8\% [see Adverse Reactions (6.1)].

Monitor patients for infusion-related reactions. For Grade 2 or 3 reactions, interrupt the infusion and provide supportive treatment. Once symptoms resolve, resume at a lower infusion rate [see Dosage and Administration (2.3)]. Administer premedication for all subsequent infusions. Discontinue BLENREP for life-threatening infusionrelated reactions and provide appropriate emergency care.

\subsection{Embryo-Fetal Toxicity}

Based on its mechanism of action, BLENREP can cause fetal harm when administered to a pregnant woman because it contains a genotoxic compound (the microtubule inhibitor, monomethyl auristatin F [MMAF]) and it targets actively dividing cells.

Advise pregnant women of the potential risk to a fetus. Advise females of reproductive potential to use effective contraception during treatment with BLENREP and for 4 months after the last dose. Advise males with female partners of reproductive potential to use effective contraception during treatment with BLENREP and for 6 months after the last dose [see Use in Specific Populations $(8.1,8.3)$ ].

\section{ADVERSE REACTIONS}

The following clinically significant adverse reactions are described elsewhere in the labeling:

- Ocular toxicity [see Warnings and Precautions (5.1)].

- Thrombocytopenia [see Warnings and Precautions (5.3)].

- Infusion-related reactions [see Warnings and Precautions (5.4)].

\subsection{Clinical Trials Experience}

Because clinical trials are conducted under widely varying conditions, adverse reaction rates observed in the clinical trials of a drug cannot be directly compared with rates in the clinical trials of another drug and may not reflect the rates observed in practice.

The pooled safety population described in Warnings and Precautions reflects exposure to BLENREP at a dosage of $2.5 \mathrm{mg} / \mathrm{kg}$ or $3.4 \mathrm{mg} / \mathrm{kg}$ (1.4 times the recommended dose) administered intravenously once every 3 weeks in 218 patients in DREAMM-2. Of these patients, 194 received a liquid formulation (not the approved dosage form) rather than the lyophilized powder. Among the 218 patients, $24 \%$ were exposed for 6 months or longer. 


\section{Relapsed or Refractory Multiple Myeloma}

The safety of BLENREP as a single agent was evaluated in DREAMM-2 [see Clinical Studies (14.1) of full Prescribing Information]. Patients received BLENREP at the recommended dosage of $2.5 \mathrm{mg} / \mathrm{kg}$ administered intravenously once every 3 weeks $(n=95)$. Among these patients, $22 \%$ were exposed for 6 months or longer.

Serious adverse reactions occurred in $40 \%$ of patients who received BLENREP. Serious adverse reactions in $>3 \%$ of patients included pneumonia $(7 \%)$, pyrexia $(6 \%)$, renal impairment $(4.2 \%)$, sepsis $(4.2 \%)$, hypercalcemia $(4.2 \%)$, and infusionrelated reactions $(3.2 \%)$. Fatal adverse reactions occurred in $3.2 \%$ of patients, including sepsis (1\%), cardiac arrest (1\%), and lung infection (1\%).

Permanent discontinuation due to an adverse reaction occurred in $8 \%$ of patients who received BLENREP; keratopathy (2.1\%) was the most frequent adverse reaction resulting in permanent discontinuation.

Dosage interruptions due to an adverse reaction occurred in $54 \%$ of patients who received BLENREP. Adverse reactions which required a dosage interruption in $>3 \%$ of patients included keratopathy $(47 \%)$, blurred vision $(5 \%)$, dry eye $(3.2 \%)$, and pneumonia (3.2\%).

Dose reductions due to an adverse reaction occurred in $29 \%$ of patients. Adverse reactions which required a dose reduction in $>3 \%$ of patients included keratopathy (23\%) and thrombocytopenia (5\%).

The most common adverse reactions ( $\geq 20 \%$ ) were keratopathy, decreased visual acuity, nausea, blurred vision, pyrexia, infusion-related reactions, and fatigue. The most common Grade 3 or $4(\geq 5 \%)$ laboratory abnormalities were lymphocytes decreased, platelets decreased, hemoglobin decreased, neutrophils decreased, creatinine increased, and gamma-glutamyl transferase increased.

Table 3 summarizes the adverse reactions in DREAMM-2 for patients who received the recommended dosage of $2.5 \mathrm{mg} / \mathrm{kg}$ once every 3 weeks.

Table 3. Adverse Reactions ( $\geq 10 \%$ ) in Patients Who Received BLENREP in DREAMM-2

\begin{tabular}{|c|c|c|}
\hline \multirow[b]{2}{*}{ Adverse Reactions } & \multicolumn{2}{|c|}{$\begin{array}{c}\text { BLENREP } \\
\mathrm{N}=95\end{array}$} \\
\hline & $\begin{array}{l}\text { All Grades } \\
(\%)\end{array}$ & $\begin{array}{c}\text { Grade 3-4 } \\
\text { (\%) }\end{array}$ \\
\hline \multicolumn{3}{|l|}{ Eye disorders } \\
\hline Keratopathy ${ }^{\mathrm{a}}$ & 71 & 44 \\
\hline Decreased visual acuity ${ }^{b}$ & 53 & 28 \\
\hline Blurred vision ${ }^{c}$ & 22 & 4 \\
\hline Dry eyes $^{d}$ & 14 & 1 \\
\hline \multicolumn{3}{|l|}{ Gastrointestinal disorders } \\
\hline Nausea & 24 & 0 \\
\hline Constipation & 13 & 0 \\
\hline Diarrhea & 13 & 1 \\
\hline \multicolumn{3}{|c|}{ General disorders and administration site conditions } \\
\hline Pyrexia & 22 & 3 \\
\hline Fatigue $^{\mathrm{e}}$ & 20 & 2 \\
\hline \multicolumn{3}{|l|}{ Procedural complications } \\
\hline Infusion-related reactions ${ }^{\dagger}$ & 21 & 3 \\
\hline \multicolumn{3}{|c|}{ Musculoskeletal and connective tissue disorders } \\
\hline Arthralgia & 12 & 0 \\
\hline Back pain & 11 & 2 \\
\hline \multicolumn{3}{|c|}{ Metabolic and nutritional disorders } \\
\hline Decreased appetite & 12 & 0 \\
\hline \multicolumn{3}{|l|}{ Infections } \\
\hline Upper respiratory tract infection ${ }^{9}$ & 11 & 0 \\
\hline
\end{tabular}

${ }^{a}$ Keratopathy was based on slit lamp eye examination, characterized as corneal epithelium changes with or without symptoms.

${ }^{b}$ Visual acuity changes were determined upon eye examination.

'Blurred vision included diplopia, vision blurred, visual acuity reduced, and visual impairment.

dDry eyes included dry eye, ocular discomfort, and eye pruritus.

${ }^{e}$ Fatigue included fatigue and asthenia.

${ }^{\dagger}$ Infusion-related reactions included infusion-related reaction, pyrexia, chills, diarrhea, nausea, asthenia, hypertension, lethargy, tachycardia.

${ }^{g}$ Upper respiratory tract infection included upper respiratory tract infection, nasopharyngitis, rhinovirus infections, and sinusitis.

Clinically relevant adverse reactions in $<10 \%$ of patients included:

Eye Disorders: Photophobia, eye irritation, infective keratitis, ulcerative keratitis.
Gastrointestinal Disorders: Vomiting.

Infections: Pneumonia

Investigations: Albuminuria.

Table 4 summarizes the laboratory abnormalities in DREAMM-2.

Table 4. Laboratory Abnormalities ( $\geq 20 \%$ ) Worsening from Baseline in Patients Who Received BLENREP in DREAMM-2

\begin{tabular}{|c|c|c|}
\hline \multirow[b]{2}{*}{ Laboratory Abnormality } & \multicolumn{2}{|c|}{$\begin{array}{l}\text { BLENREP } \\
\mathrm{N}=95\end{array}$} \\
\hline & $\begin{array}{l}\text { All Grades } \\
\text { (\%) }\end{array}$ & $\begin{array}{c}\text { Grades 3-4 } \\
(\%)\end{array}$ \\
\hline \multicolumn{3}{|l|}{ Hematology } \\
\hline Platelets decreased & 62 & 21 \\
\hline Lymphocytes decreased & 49 & 22 \\
\hline Hemoglobin decreased & 32 & 18 \\
\hline Neutrophils decreased & 28 & 9 \\
\hline \multicolumn{3}{|l|}{ Chemistry } \\
\hline $\begin{array}{l}\text { Aspartate aminotransferase } \\
\text { increased }\end{array}$ & 57 & 2 \\
\hline Albumin decreased & 43 & 4 \\
\hline Glucose increased & 38 & 3 \\
\hline Creatinine increased & 28 & 5 \\
\hline Alkaline phosphatase increased & 26 & 1 \\
\hline $\begin{array}{l}\text { Gamma-glutamyl transferase } \\
\text { increased }\end{array}$ & 25 & 5 \\
\hline Creatinine phosphokinase increased & 22 & 1 \\
\hline Sodium decreased & 21 & 2 \\
\hline Potassium decreased & 20 & 2 \\
\hline
\end{tabular}

\subsection{Immunogenicity}

As with all therapeutic proteins, there is potential for immunogenicity. The detection of antibody formation is highly dependent on the sensitivity and specificity of the assay. Additionally, the observed incidence of antibody (including neutralizing antibody) positivity in an assay may be influenced by several factors including assay methodology, sample handling, timing of sample collection, concomitant medications, and underlying disease. For these reasons, comparison of the incidence of antibodies in the studies described below with the incidence of antibodies in other studies or to other products may be misleading.

The immunogenicity of BLENREP was evaluated using an electrochemiluminescence (ECL)-based immunoassay to test for anti-belantamab mafodotin antibodies. In clinical studies of BLENREP, 2/274 patients $(<1 \%)$ tested positive for antibelantamab mafodotin antibodies after treatment. One of the 2 patients tested positive for neutralizing anti-belantamab mafodotin antibodies following 4 weeks on therapy. Due to the limited number of patients with antibodies against belantamab mafodotin-blmf, no conclusions can be drawn concerning a potential effect of immunogenicity on pharmacokinetics, efficacy, or safety.

\section{USE IN SPECIFIC POPULATIONS \\ 8.1 Pregnancy \\ Risk Summary}

Based on its mechanism of action, BLENREP can cause fetal harm when administered to a pregnant woman, because it contains a genotoxic compound (the microtubule inhibitor, MMAF) and it targets actively dividing cells [see Clinical Pharmacology (12.1), Nonclinical Toxicology (13.1) of full Prescribing Information]. Human immunoglobulin $\mathrm{G}(\mathrm{lgG})$ is known to cross the placenta; therefore, belantamab mafodotin-blmf has the potential to be transmitted from the mother to the developing fetus. There are no available data on the use of BLENREP in pregnant women to evaluate for drug-associated risk. No animal reproduction studies were conducted with BLENREP. Advise pregnant women of the potential risk to a fetus.

The estimated background risk of major birth defects and miscarriage for the indicated population is unknown. All pregnancies have a background risk of birth defect, loss, or other adverse outcome. In the U.S. general population, the estimated background risk of major birth defects and miscarriage in clinically recognized pregnancies is $2 \%$ to $4 \%$ and $15 \%$ to $20 \%$, respectively.

Data

Animal Data: Animal reproductive or developmental toxicity studies were not conducted with belantamab mafodotin-blmf. The cytotoxic component of BLENREP, MMAF, disrupts microtubule function, is genotoxic, and can be toxic to rapidly dividing cells, suggesting it has the potential to cause embryotoxicity and teratogenicity. 


\subsection{Lactation}

\section{Risk Summary}

There is no data on the presence of belantamab mafodotin-blmf in human milk or the effects on the breastfed child or milk production. Because of the potential for serious adverse reactions in the breastfed child, advise women not to breastfeed during treatment with BLENREP and for 3 months after the last dose.

\subsection{Females and Males of Reproductive Potential}

BLENREP can cause fetal harm when administered to pregnant women [see Use in Specific Populations (8.1)].

\section{Pregnancy Testing}

Pregnancy testing is recommended for females of reproductive potential prior to initiating BLENREP.

\section{Contraception}

Females: Advise women of reproductive potential to use effective contraception during treatment and for 4 months after the last dose.

Males: Because of the potential for genotoxicity, advise males with female partners of reproductive potential to use effective contraception during treatment with BLENREP and for 6 months after the last dose [see Nonclinical Toxicology (13.1) of full Prescribing Information].

\section{Infertility}

Based on findings in animal studies, BLENREP may impair fertility in females and males. The effects were not reversible in male rats, but were reversible in female rats [see Nonclinical Toxicology (13.1) of full Prescribing Information].

\subsection{Pediatric Use}

The safety and effectiveness of BLENREP in pediatric patients have not been established.

\subsection{Geriatric Use}

Of the 218 patients who received BLENREP in DREAMM-2, 43\% were aged 65 to less than 75 years and $17 \%$ were aged 75 years and older. Clinical studies of BLENREP did not include sufficient numbers of patients aged 65 and older to determine whether the effectiveness differs compared with that of younger patients. Keratopathy occurred in $80 \%$ of patients aged less than 65 years and $73 \%$ of patients aged 65 years and older. Among the patients who received BLENREP at the $2.5-\mathrm{mg} / \mathrm{kg}$ dose in DREAMM-2 $(\mathrm{n}=95)$, keratopathy occurred in $67 \%$ of patients aged less than 65 years and $73 \%$ of patients aged 65 years and older. Clinical studies did not include sufficient numbers of patients 75 years and older to determine whether they respond differently compared with younger patients.

\subsection{Renal Impairment}

No dose adjustment is recommended for patients with mild or moderate renal impairment (estimated glomerular filtration rate [eGFR] 30 to $89 \mathrm{~mL} / \mathrm{min} / 1.73 \mathrm{~m}^{2}$ as estimated by the Modification of Diet in Renal Disease [MDRD] equation) [see Clinical Pharmacology (12.3) of full Prescribing Information]. The recommended dosage has not been established in patients with severe renal impairment (eGFR 15 to $29 \mathrm{~mL} / \mathrm{min} / 1.73 \mathrm{~m}^{2}$ ) or end-stage renal disease (ESRD) with eGFR $<15 \mathrm{~mL}$ $\mathrm{min} / 1.73 \mathrm{~m}^{2}$ not on dialysis or requiring dialysis [see Clinical Pharmacology (12.3) of full Prescribing Information].

\subsection{Hepatic Impairment}

No dose adjustment is recommended for patients with mild hepatic impairment (total bilirubin $\leq$ upper limit of normal [ULN] and aspartate aminotransferase (AST) $>$ ULN or total bilirubin 1 to $\leq 1.5 \times$ ULN and any AST).

The recommended dosage of BLENREP has not been established in patients with moderate or severe hepatic impairment (total bilirubin $>1.5 \times$ ULN and any AST) [see Clinical Pharmacology (12.3) of full Prescribing Information].

\section{REFERENCES}

1. "OSHA Hazardous Drugs." OSHA.

http://www.osha.gov/SLTC/hazardousdrugs/index.html.

\section{PATIENT COUNSELING INFORMATION}

Advise the patient to read the FDA-approved patient labeling (Medication Guide).

\section{Ocular Toxicity}

- Advise patients that ocular toxicity may occur during treatment with BLENREP [see Warnings and Precautions (5.1)].

- Advise patients to administer preservative-free lubricant eye drops as recommended during treatment and to avoid wearing contact lenses during treatment unless directed by a healthcare professional [see Dosage and Administration (2.3), Warnings and Precautions (5.1)].

- Advise patients to use caution when driving or operating machinery as BLENREP may adversely affect their vision [see Warnings and Precautions (5.1)].

\section{BLENREP REMS}

BLENREP is available only through a restricted program called BLENREP REMS [see Warnings and Precautions (5.2)]. Inform the patient of the following notable requirements:

- Patients must complete the enrollment form with their provider.

- Patients must comply with ongoing monitoring for eye exams [see Warnings and Precautions (5.1)].

Thrombocytopenia

- Advise patients to inform their healthcare provider if they develop signs or symptoms of bleeding [see Warnings and Precautions (5.3)].

\section{Infusion-Related Reactions}

- Advise patients to immediately report any signs and symptoms of infusion-related reactions to their healthcare provider [see Warnings and Precautions (5.4)].

Embryo-Fetal Toxicity

- Advise pregnant women of the potential risk to a fetus. Advise females of reproductive potential to inform their healthcare provider of a known or suspected pregnancy [see Warnings and Precautions (5.5), Use in Specific Populations (8.1, 8.3)].

- Advise women of reproductive potential to use highly effective contraception during treatment and for 4 months after the last dose [see Warnings and Precautions (5.5), Use in Specific Populations (8.3)].

- Advise males with female partners of reproductive potential to use effective contraception during treatment with BLENREP and for 6 months after the last dose [see Use in Specific Populations (8.3), Nonclinical Toxicology (13.1) of full Prescribing Information].

\section{Lactation}

- Advise women not to breastfeed during treatment with BLENREP and for 3 months after the last dose [see Use in Specific Populations (8.2)].

Infertility

- Advise males and females of reproductive potential that BLENREP may impair fertility [see Use in Specific Populations (8.3)].

Trademarks are owned by or licensed to the GSK group of companies.

Manufactured by:

GlaxoSmithKline Intellectual Property Development Ltd. England

Brentford, Middlesex, UK TW8 9GS

U.S. License No. 2148

including by use of Potelligent technology licensed from BioWa, Inc.

For:

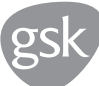

GlaxoSmithKline

Research Triangle Park, NC 27709

(C)2020 GSK group of companies or its licensor.

August 2020 BRP:1BRS

(C)2020 GSK or licensor.

BLMJRNA200002 August 2020

Produced in USA. 


\section{Review Article: ADCs in Lymphoma}

Continued from page 524

leading to an expanded label, came from the AETHERA study, which tested BV consolidation in patients undergoing autoSCT for relapsed high-risk HL. ${ }^{27}$ A significant improvement in PFS was demonstrated in patients receiving $\mathrm{BV}$ (5-year PFS of $59 \%$ vs $41 \%$ in those receiving placebo), which has led to a new standard of care in this patient population. Peripheral neuropathy led 23\% of patients to discontinue treatment prior to completing all planned 16 cycles, and another $31 \%$ required dose modifications or delays, revealing the difficulties with long-term use of this agent. BV is now also approved for use in frontline HL management. The multicenter, randomized, open-label, phase 3 ECHELON-1 study explored BV plus doxorubicin, vinblastine, and dacarbazine, compared with the standard-of-care of bleomycin plus the same latter 3 agents (ABVD) in the frontline HL setting. ${ }^{28}$ Modified PFS at 3 years was superior in patients receiving BV plus chemotherapy (83\%) compared with those who received ABVD (76\%). How BV-containing regimens are integrated with modern PET-adapted treatment strategies is still evolving.

CD30 is also expressed in a portion of cases of peripheral $\mathrm{T}$ cell lymphoma (PTCL) not otherwise specified, angioimmunoblastic T-cell lymphoma, adult T-cell leukemia/lymphoma, and cutaneous T-cell lymphoma (CTCL) including Sezary syndrome and mycosis fungoides (MF). ${ }^{22}$ The landmark multicenter, randomized, double-blind, phase 2 ECHELON-2 study ${ }^{29}$ in untreated patients with CD30-positive PTCL demonstrated a survival advantage with the addition of BV to frontline chemotherapy, improving median PFS from 20.8 to 48.2 months and reducing the risk of death by $34 \%$. Similarly, the phase 3 ALCANZA trial ${ }^{30}$ compared BV with physician's choice of therapy (methotrexate or bexarotene) in the treatment of R/R CD30-positive CTCL, with an objective global response being seen in $56.3 \%$ of patients receiving BV compared with $12.5 \%$ of those on the physician's-choice arm. BV is now indicated for use in the settings of untreated HL, R/R HL, HL after autoSCT, R/R MF, untreated PTCL, untreated ALCL, and R/R ALCL. When used in combination with chemotherapy for untreated lymphomas, G-CSF prophylaxis is indicated. ${ }^{24}$

Interestingly, there are provocative data regarding the correlation between CD30 expression and responses to BV. One analysis integrated data from 5 prospective clinical trials that included 275 patients with B- and T-cell lymphomas treated with BV. ${ }^{31}$ Of these, 143 had tumors with CD30 expression of $<10 \%$, including 58 of the 143 who had undetectable CD30 by this independent review. BV activity was observed at all levels of CD30 expression; the ORR was 34\% (20/58) in cases with undetectable CD30. While hypothesis-generating at this point, these observations raise important questions regarding the methodology of assessing heterogenous CD30 expression on lymphomas, as well as potential off-target consequences of BV such as bystander effects within tumors with low or undetectable CD30 expression.

\section{Polatuzumab Vedotin}

Polatuzumab vedotin (Polivy; PV) was the second ADC to receive approval for the treatment of lymphoma. It is composed of a humanized anti-CD79b IgG1 mAb, and it mimics BV in its use of the same protease-cleavable linker to affix the microtubule-targeting MMAE payload. ${ }^{32}$ Together, CD79a and CD79b form the heterodimeric signaling component of the B-cell receptor (BCR). Crosslinking of the BCR by antigens or antibodies triggers internalization and trafficking of the complex to the lysosomal-like major histocompatibility complex (MHC) class II endocytic compartment. This internalization, combined with the restriction of CD79b expression to the B-cell lineage, makes CD79b an attractive candidate target for ADC development.

The multicenter, open-label, phase 1 study of PV enrolled patients with B-cell non-Hodgkin lymphoma (NHL) or chronic lymphocytic leukemia (CLL) who had R/R disease or no available curative treatment options. ${ }^{33} \mathrm{PV}$ was dosed between 0.1 and $2.4 \mathrm{mg} / \mathrm{kg}$ every 21 days, and the MTD in NHL was 2.4 $\mathrm{mg} / \mathrm{kg}$. The ORR in patients with NHL treated at the PV MTD was 51\% (23/45), with median PFS and DOR of 5.7 and 6.2 months, respectively. There were also expansion cohorts for patients with NHL to combine rituximab with PV at the MTD. However, no expansion CLL cohort was pursued due to lack of activity in the PV monotherapy dose-escalation CLL cohort. On the whole, the most common grade 3 or higher AEs were neutropenia, anemia, and peripheral neuropathy, perhaps aligning in part with what was seen in trials of BV due to a common cytotoxic payload. Notably, $51 \%$ of patients $(23 / 45)$ discontinued PV treatment due to an $\mathrm{AE}$, including discontinuation due to peripheral neuropathy in $24 \%$ of patients $(11 / 45)$. One part of the subsequent multicenter, open-label, phase 2 ROMULUS study explored the combination of rituximab with $\mathrm{PV}$ at the $2.4 \mathrm{mg} / \mathrm{kg}$ MTD in a larger cohort. ${ }^{34}$ Promising ORRs of $54 \%$ and $70 \%$ were seen in both the R/R diffuse large B-cell lymphoma (DLBCL) and follicular lymphoma (FL) settings, respectively. These findings motivated further PV combination studies. In contrast, the other arm of the ROMULUS study explored pinatuzumab vedotin, a similarly designed ADC targeting CD22, but due to shorter DOR, shorter PFS, and nine grade 5 AEs occurring in patients receiving pinatuzumab vedotin, this anti-CD22 ADC was not recommended for further study at that time. 


\section{TABLE. Notable Active/Recruiting Clinical Trials of ADCs in Development for Lymphoma}

\begin{tabular}{|c|c|c|c|c|c|c|c|}
\hline ADC Name (sponsor) & Target & $\begin{array}{l}\text { Additional } \\
\text { Agents }\end{array}$ & Phase & NCT Number & Eligible Histologies & $\begin{array}{l}\text { Start } \\
\text { Date }\end{array}$ & $\begin{array}{l}\text { Completion } \\
\text { Date }\end{array}$ \\
\hline \multirow{4}{*}{$\begin{array}{c}\text { Loncastuximab } \\
\text { tesirine (Lonca, ADCT- } \\
402 ; \\
\text { ADC Therapeutics) }\end{array}$} & \multirow{4}{*}{ CD19 } & None & 2 & NCT03589469 & R/R DLBCL & August 2018 & March 2021 \\
\hline & & Durvalumab & 1 & NCT03685344 & R/R DLBCL, MCL, FL & $\begin{array}{l}\text { December } \\
\quad 2018\end{array}$ & January 2021 \\
\hline & & Ibrutinib & $1 / 2$ & NCT03684694 & R/R DLBCL, MCL & $\begin{array}{l}\text { December } \\
2018\end{array}$ & April 2023 \\
\hline & & $\begin{array}{l}\text { Rituximab, } \\
\text { gemcitabine, } \\
\text { oxaliplatin }\end{array}$ & 3 & NCT04384484 & R/R DLBCL & $\begin{array}{c}\text { September } \\
2020\end{array}$ & $\begin{array}{c}\text { December } \\
2024\end{array}$ \\
\hline \multirow{3}{*}{$\begin{array}{c}\text { MT-3724 } \\
\text { (Molecular Templates) }\end{array}$} & \multirow{3}{*}{ CD20 } & None & $1 / 2$ & NCT02361346 & R/R B-cell NHL, CLL & $\begin{array}{l}\text { February } \\
2015\end{array}$ & $\begin{array}{l}\text { September } \\
\quad 2021\end{array}$ \\
\hline & & $\begin{array}{l}\text { Gemcitabine, } \\
\text { oxaliplatin }\end{array}$ & 2 & NCT03488251 & R/R B-cell NHL & August 2018 & February 2023 \\
\hline & & Lenalidomide & 2 & NCT03645395 & R/R B-cell NHL & April 2019 & $\begin{array}{l}\text { July } \\
2022\end{array}$ \\
\hline $\begin{array}{l}\text { TRPH-222 } \\
\text { (Triphase) }\end{array}$ & CD22 & None & 1 & NCT03682796 & $\begin{array}{c}\text { R/R DLBCL, MCL, FL, } \\
\text { MZL }\end{array}$ & $\begin{array}{l}\text { October } \\
2018\end{array}$ & August 2022 \\
\hline $\begin{array}{c}\text { Camidanlumab } \\
\text { tesirine (Cami, ADCT- } \\
301 ; \\
\text { ADC Therapeutics) }\end{array}$ & CD25 & None & 2 & NCT04052997 & $\mathrm{R} / \mathrm{R} \mathrm{HL}$ & $\begin{array}{l}\text { September } \\
2019\end{array}$ & May 2024 \\
\hline $\begin{array}{c}\text { Naratuximab } \\
\text { emtansine } \\
\text { (Debio1562, } \\
\text { IMGN592; } \\
\text { Debiopharm) }\end{array}$ & CD37 & Rituximab & 2 & NCT02564744 & $\begin{array}{c}\text { R/R DLBCL, MCL, FL, } \\
\text { MZL }\end{array}$ & June 2016 & January 2021 \\
\hline $\begin{array}{c}\text { STRO-001 } \\
\text { (Sutro Biopharma) }\end{array}$ & CD74 & None & 1 & NCT03424603 & R/R B-cell NHL, MM & $\begin{array}{l}\text { February } \\
2018\end{array}$ & $\begin{array}{l}\text { November } \\
2023\end{array}$ \\
\hline $\begin{array}{l}\text { VLS-101 } \\
\text { (VelosBio) }\end{array}$ & ROR1 & None & 1 & NCT03833180 & $\begin{array}{l}\text { R/R B cell NHL, CLL, } \\
\text { Richter transformation, } \\
\text { T-cell NHL, ALL, } \\
\text { AML, Waldenström } \\
\text { macroglobulinemia }\end{array}$ & $\begin{array}{l}\text { February } \\
2019\end{array}$ & June 2021 \\
\hline
\end{tabular}

ADC, antibody-drug conjugate; ALL, acute lymphocytic leukemia; AML, acute myeloid leukemia; CLL, chronic lymphocytic leukemia; DLBCL, diffuse large B-cell lymphoma; FL, follicular lymphoma; HL, Hodgkin lymphoma; MCL, mantle cell lymphoma; MM, multiple myeloma; MZL, marginal zone lymphoma; NHL, non-Hodgkin lymphoma; R/R, relapsed or refractory.

Recently, the results of an important phase $1 \mathrm{~b} / 2$ study of PV have provided important insight into how and where in the lymphoma treatment algorithm this agent should be used. Prior studies as well as exposure-response and timeto-event analyses revealed the cumulative nature of peripheral neuropathy leading to PV discontinuations. ${ }^{35}$ In turn, dosing of PV was limited to $1.8 \mathrm{mg} / \mathrm{kg}$. This multiarm study ${ }^{36}$ explored PV combined with the alkylating agent bendamustine plus the second-generation anti-CD20
mAb obinutuzumab (Gazyva). It also explored a randomized comparison of PV plus bendamustine and rituximab (BR) vs BR alone, in the autoSCT-ineligible $\mathrm{R} / \mathrm{R}$ DLBCL setting. Impressively, in the randomly assigned cohort (40 patients per arm), patients receiving PV with BR had a significantly higher CR rate $(40 \%$ vs $17 \%$ ), as well as longer median PFS (9.5 vs 3.7 months) and median OS (12.4 vs 4.7 months) compared with patients receiving $\mathrm{BR}$ alone. Of the responses to PV with BR, 64\% (16/25) lasted at least
6 months and $48 \%(12 / 25)$ lasted at least 12 months. Rates of neutropenia, anemia, and thrombocytopenia were higher in patients receiving $\mathrm{PV}$ in addition to $\mathrm{BR}$, but grade 3 to 4 infection rates were similar between the 2 cohorts. Peripheral neuropathy associated with PV occurred in $43 \%$ of patients but was typically grade 1 to 2 and resolved in most patients. In turn, PV was approved for use with BR under the FDA's accelerated approval program for the R/R DLBCL setting. Of note, prophylaxis for both Pneumocystis 
jiroveci pneumonia and herpes virus should be given throughout treatment with this regimen. ${ }^{37}$

In parallel, PV was studied in a phase $1 b / 2$ trial in combination with either rituximab or obinutuzumab, plus cyclophosphamide, doxorubicin, and prednisone $(\mathrm{CHP})$ in patients with previously untreated DLBCL..$^{38}$ In 66 patients who received $\mathrm{PV}$ at $1.8 \mathrm{mg} / \mathrm{kg}$, hematologic toxicities and peripheral neuropathy were common but occurred at expected rates. The ORR was $89 \%(59 / 66)$ with $77 \%$ of patients achieving CR. Ongoing exploration of $\mathrm{PV}$ includes the follow-up phase 3 POLARIX study of PV plus rituximab $(\mathrm{R})$ and $\mathrm{CHP}$ vs traditional frontline $\mathrm{R}-\mathrm{CHOP}$ (CHP plus vincristine) in untreated DLBCL, the phase 3 POLAR BEAR study of PV plus R-mini-CHP vs R-mini-CHOP in elderly and/or frail patients with untreated DLBCL, as well as studies of $\mathrm{PV}$ in combination with atezolizumab (Tecentriq; anti-PD-L1) or mosunetuzumab (an anti-CD3/CD20 bispecific antibody).

\section{ADCs in Development for Lymphoma CD19}

Loncastuximab tesirine (lonca or ADCT402) is an ADC targeting CD19, an antigen expressed early in B-cell development starting at the pro-B-cell stage, making this an attractive target for both B-cell lymphoblastic leukemia as well as lymphomas derived from B-cells through the late plasmablast stage..$^{39}$ This agent incorporates a humanized anti-CD19 IgG1 $\mathrm{mAb}$ and a PBD dimer payload attached via a protease-cleavable valine-alanine linker. ${ }^{40} \mathrm{~A}$ multicenter, open-label, phase 1 dose-escalation (15 to $200 \mu \mathrm{g} / \mathrm{kg}$ ) and dose-expansion study of lonca enrolled 88 patients with R/R B-cell NHL. ${ }^{41}$ Common AEs included cytopenias, fatigue, edema, liver test abnormalities, nausea, rash, and dyspnea. The ORR at doses $>120 \mu \mathrm{g} / \mathrm{kg}$ was $59 \%$ (41/69), with $40 \% \mathrm{CR}$ and $19 \%$ PR rates. Median DOR, PFS, and OS were $4.8,5.5$, and 11.6 months, respectively.
There was moderate drug accumulation with higher doses, but no evidence of immunogenicity. The subsequent multicenter, open-label, phase 2 LOTIS-2 study treated 145 patients with $\mathrm{R} / \mathrm{R}$ DLBCL, high-grade B-cell lymphoma, or primary mediastinal B-cell lymphoma with lonca monotherapy dosed at 150 $\mu \mathrm{g} / \mathrm{kg}$ every 3 weeks for 2 cycles, then $75 \mu \mathrm{g} / \mathrm{kg}$ every 3 weeks for subsequent cycles for up to 1 year..$^{42}$ Data from this study were presented in mid-2020, and they observed an ORR of $48 \%$ (70/145), with $24 \%$ CR and $24 \%$ PR; median DOR was 10.3 months. These promising results motivated a September 2020 submission of a biologics license application to the FDA for lonca for the treatment of patients with R/R DLBCL. Two additional ongoing phase 1 studies are exploring lonca in combination with either ibrutinib (Imbruvica; an oral Bruton tyrosine kinase inhibitor) or durvalumab (Darzalex; anti-PD-L1). Finally, a phase 3 study has been announced that will incorporate a safety run-in to explore combining lonca with rituximab; it will then randomize patients with R/R DLBCL to lonca plus rituximab vs chemotherapy (gemcitabine and oxaliplatin) with rituximab.

Less successful than lonca has been an alternative anti-CD19 ADC agent, coltuximab ravtansine (SAR3419). It incorporates a humanized anti-CD19 IgG1 $\mathrm{mAb}$ with DM4 (a microtubule-targeting maytansine derivative) payload, linked via an N-succinimidyl-4-(2-pyridyldithio) butyrate glutathione-sensitive linker. ${ }^{43}$ The initial phase 1 study of SAR3419 (administered weekly at doses between 5 and $70 \mathrm{mg} / \mathrm{m}^{2}$ ) observed episodes of grade 3 optic neuropathy and grade 3 paresthesia, which were reversible but led to treatment discontinuation. ${ }^{44}$ Due to this and other clinical evidence of cumulative toxicities, the study transitioned to a dosing schedule of 4 weekly doses followed by 4 biweekly doses. The ORR was $30 \%$ in the total cohort, with dosing schema not significantly impacting response rate. Two phase 2 studies explored SAR3419 in
R/R DLBCL, either as a monotherapy or in combination with rituximab. ${ }^{45,46}$ In the monotherapy group, the ORR was $44 \%$ (18/41) with median DOR, PFS, and OS of 4.7, 4.4, and 9.2 months, respectively. Grade 3-4 AEs were reported in 38\% of patients, and eye disorders (all grade $1-2)$ occurred in $25 \%$ of patients. The combination of rituximab and SAR3419 in the R/R DLBCL setting failed to meet its primary end point, observing an ORR of $31 \%$. Likely due to the modest clinical activity of this agent and the various toxicities, there are no ongoing clinical trials for SAR3419.

Denintuzumab mafodotin (SGNCD19A) is another ADC targeting CD19 and it incorporates an MMAE payload. It was studied in a phase 1 trial $^{47}$ in R/R B-cell NHL, and the MTD was not reached ( 0.5 to $6 \mathrm{mg} / \mathrm{kg}$ every 21 days). Only 1 DLT was observed (grade 3 keratopathy at $3 \mathrm{mg} / \mathrm{kg}$ ), but the reported AEs included blurry vision (65\%), dry eye $(52 \%)$, fatigue $(35 \%)$, keratopathy (35\%), constipation (29\%), photophobia $(27 \%)$, and nausea (26\%). Overall, $33 \%$ (20/60) of efficacy-evaluable patients achieved objective responses, including $22 \%(13 / 60)$ with CRs. Randomized phase 2 trials to evaluate rituximab plus ifosfamide, carboplatin, and etoposide salvage treatment with or without SGNCD19A were opened but later terminated due to sponsor decision based on portfolio prioritization.

\section{CD20}

MT-3724 is an ADC specifically targeting CD20, which is expressed from the pre-B-cell stage through the plasmablast stage, and this agent possesses multiple unique design features. Specifically, in contrast to the common intact IgG mAb, MT-3724 utilizes a single-chain variable fragment $(\mathrm{scFv})$, and it is connected via a peptide linker to an irreversible ribosome inhibitor derived from the Shiga-like toxin (SLT) A subunit. CD20 is a noninternalized surface antigen, thus MT-3724 exploits proprietary engineered toxin 
bodies technology to achieve intracellular localization..$^{48}$ Specifically, SLTA is normally coupled to its cognate SLTB subunit to target the CD77 cell-surface marker, a noninternalizing glycosphingolipid. In MT-3724, a genetically engineered SLTA subunit with no cognate SLTB component is able to trigger spontaneous internalization, and it then goes on to inhibit protein translation. A multicenter, open-label, phase 1 dose-escalation and dose-expansion study of MT-3724 monotherapy in patients with R/R NHL is ongoing. ${ }^{49}$ Preliminary analyses reported an ORR of $38 \%$. Interestingly, the development of ADAs did not preclude benefit of MT3724. Other ongoing studies of MT-3724 include phase 2 trials combining this agent with gemcitabine and oxaliplatin chemotherapy, or with lenalidomide, in the second-line, autoSCT-ineligible, R/R DLBCL setting. An unplanned interim analysis of the study of MT-3724 plus lenalidomide found grade 2 capillary leak syndrome was occurring at lower doses of MT-3724 than expected. ${ }^{50}$ Thus, a modified MT-3724 dosing schedule (twice weekly rather than 3 times weekly) is under development. Preliminary response data revealed 2 CRs and 3 PRs in the 7 evaluable patients.

\section{CD22}

CD22 is a member of the sialic acid-binding immunoglobulin-like lectin receptor family, and it is expressed on the surface of immature and mature B-cells. It plays a critical role in baseline BCR signaling inhibition and is rapidly internalized upon antibody binding. Inotuzumab ozogamicin is an ADC that targets CD22 with a humanized IgG4 mAb that uses an acid-labile covalent linker to attach a semisynthetic calicheamicin derivative that binds DNA and triggers strand scission. ${ }^{51}$ Inotuzumab ozogamicin monotherapy is approved for $\mathrm{R} / \mathrm{R} \mathrm{B}$-cell acute lymphoblastic leukemia, and it is also being increasingly incorporated off-label into lower-intensity frontline combination approaches for patients who are less fit. ${ }^{52-54}$ Its study in lymphoma has shown mixed results. A phase 1 study $^{55}$ of inotuzumab ozogamicin in the R/R B-cell NHL setting tested doses between 0.4 and $2.4 \mathrm{mg} / \mathrm{m}^{2}$, administered once every 3 or 4 weeks. The MTD was determined to be $1.8 \mathrm{mg} / \mathrm{m}^{2}$, and common AEs included thrombocytopenia (90\%), asthenia $(67 \%)$, nausea $(51 \%)$, and neutropenia (51\%). The ORR was $39 \%$ (68\% for FL; $15 \%$ for DLBCL) for the 79 enrolled patients. Among the follow-up studies, a phase 2 trial administered inotuzumab ozogamicin with rituximab every 3 weeks for 3 planned cycles (but up to 6 were permitted), followed by high-dose therapy and autoSCT in patients with high-risk R/R DLBCL. ${ }^{56}$ Sixty-three patients enrolled, and the ORR after 3 cycles (the primary end point) was $28.6 \%$. Eighteen patients underwent autoSCT, with serious infections and hepatic toxicity following autoSCT occurring in $33 \%$ and $22 \%$, respectively. The 1 -year PFS for all enrolled patients was 29\% (median PFS, 3.0 months), thus showing lower-than-expected activity as a salvage regimen for transplant-eligible patients with DLBCL.

Another anti-CD22 ADC that is relatively early in development is TRPH222. This agent incorporates a mAb containing formylglycine modifications to facilitate site-specific conjugation of a maytansinoid antimicrotubule payload via a noncleavable, protease-insensitive linker. ${ }^{57} \mathrm{~A}$ multicenter, open-label, phase 1 study with dose escalation and dose expansion phases in $\mathrm{R} / \mathrm{R}$ B-cell NHL is ongoing. TRPH-222 was dosed between 0.6 and $5.6 \mathrm{mg} / \mathrm{kg}$ every 21 days, and $26 \%(5 / 19)$ of evaluable patients achieved CR without reaching the MTD. In turn, a $10 \mathrm{mg} / \mathrm{kg}$ dosing cohort is being explored. Notably, peripheral neuropathy, which is common with antimicrotubule ADCs, has not been observed in the patients to date.

\section{CD25}

CD25, the interleukin-2 receptor alpha, is expressed on many tissues; these include activated and regulatory $\mathrm{T}$ cells, activated B cells, a subset of thymocytes, myeloid precursors, and oligodendrocytes. It is overexpressed in a variety of malignant settings; therefore, camidanlumab tesirine (cami or ADCT-301) has been developed to combine a human anti-CD25 IgG1 that is conjugated via a dipeptide cleavable linker to a PBD dimer payload.58 In vitro and in vivo studies found high potency of this agent, with significant bystander killing of CD25-negative cells. An initial multicenter, open-label, phase 1 study explored ADCT-301 in patients with R/R HL and NHL that express CD25. ${ }^{59-61}$ Of a total of 77 treated patients, $65 \%$ had skin-related AEs; other AEs included liver function test abnormalities (39\%), edema $(25 \%)$, peripheral neuropathy $(9 \%)$, and Guillain-Barre syndrome (GBS) and radiculopathy (6\% each). In the 75 evaluable patients, ORR was $70.7 \%$ (53/75), with CR in 40 patients; in addition, the response rate was an impressive $93 \%$ in patients with R/R HL relapsing within 6 months of immune checkpoint inhibitor treatment. Based on this phase 1 study, the recommended phase 2 dose was 45 $\mu \mathrm{g} / \mathrm{kg}$ every 3 weeks for 2 cycles followed by $30 \mu \mathrm{g} / \mathrm{kg}$ every 3 weeks. This phase 2 study was reopened for enrollment after being placed under a partial clinical hold to allow for exploration of the atypical toxicities, including GBS, from the phase 1 study.

\section{CD37}

CD37 is a member of the tetraspanin family and mediates aspects of immune response signal transduction. ${ }^{62}$ Its expression is restricted to hematopoietic cells, and it is most robustly found on mature B cells, with CD37 loss leading to spontaneous lymphomagenesis in a mouse model. Naratuximab emtansine (IMGN529 or Debio1562) is an ADC that incorporates a humanized $\operatorname{IgG} 1$ anti-CD37 $\mathrm{mAb}$, which on its own exhibits significant $\mathrm{ADCC}$ and $\mathrm{CDC} .{ }^{63}$ It uses a thioether linker that can undergo lysosomal cleavage to carry the 
maytansinoid-derivative DM1 payload. IMGN529 was explored in a multicenter, open-label, phase 1 trial $^{64}$ in patients with R/R B-cell NHL. Of 49 patients receiving doses escalating from 0.1 to $1.8 \mathrm{mg} / \mathrm{kg}$, DLTs included peripheral neuropathy, neutropenia, and thrombocytopenia. AEs led to treatment discontinuation in $20 \%$ of patients $(10 / 50)$, with $16 \%(8 / 50)$ having serious treatment-related AEs, the most common being grade 3 febrile neutropenia. The MTD was determined to be $1.4 \mathrm{mg} / \mathrm{kg}$ every 3 weeks, with growth factor support being required. An objective response was achieved by $13 \%(5 / 39)$ of response-evaluable patients . After Debiopharm acquired IMGN529 from ImmunoGen, it was renamed Debio1562, and now a phase 2 study of this agent in combination with rituximab is underway.

\section{CD70}

CD70 is the ligand of the TNF receptor family member $\mathrm{CD} 27$, and it is expressed on a broad range of malignancies including lymphomas (about $50 \%$ to $60 \%$ of NHL). ${ }^{65} \mathrm{SGN}-\mathrm{CD} 70 \mathrm{~A}$ is an ADC incorporating an anti-CD70 $\mathrm{mAb}$ with a protease-cleavable linker and a DNA-crosslinking PBD dimer payload. This agent has been studied only in a phase 1 trial including patients with relapsed renal cell carcinoma, mantle cell lymphoma (MCL), DLBCL, and FL grade $3 .{ }^{66}$ It was administered at dose levels ranging from 8 to $200 \mu \mathrm{g} / \mathrm{kg}$ every 21 days, but because prolonged thrombocytopenia was observed, the study was amended to dose SGN-CD70A every 6 weeks. Overall, 20 patients with lymphoma were enrolled and treated, with an MTD of $30 \mu \mathrm{g} / \mathrm{kg}$ every 6 weeks being determined. Common AEs included thrombocytopenia $(75 \%)$, nausea $(55 \%)$, anemia $(50 \%)$, and fatigue $(50 \%)$. Responses were seen only in 20\% (4/20) of patients. Due to very modest single-agent activity and the frequency and severity of thrombocytopenia, no subsequent studies have been opened with this agent.

\section{CD74}

CD74 is a multifunctional protein that serves as an MHC class II chaperone; it partially localizes to the cell surface, where it is a receptor for macrophage migration inhibitory factor as well as a regulator of B-cell survival. ${ }^{67}$ It is highly expressed on various $\mathrm{B}$-cell malignancies, and STRO-001 is a novel ADC that incorporates an anti-CD74 $\mathrm{mAb}$ that uses a noncleavable linker to achieve site-specific attachment of 2 maytansinoid payloads. ${ }^{68}$ An initial phase 1 trial that includes patients with $\mathrm{R} / \mathrm{R} \mathrm{B}$ cell NHL and multiple myeloma is underway. Preliminary results described common infusion reactions and 2 DLTs (one grade 3 and one grade 5 thromboembolic event, resulting in a protocol amendment requiring screening for thrombosis at baseline). No ocular toxicity signals had been observed and the MTD had not been reached. Both CRs and PRs have been observed in patients with DLBCL.

\section{ROR1}

ROR1, a transmembrane receptor, has been found to be expressed on multiple tumor types but not on normal adult tissues. ${ }^{69}$ DLBCL, CLL, and MCL commonly express ROR1, motivating the development of VLS-101, an ADC that links an MMAE payload to the mAb cirmtuzumab. This agent incorporates a highly specific, humanized, IgG1 mAb that triggers rapid internalization and trafficking to lysosomes. A phase 1 clinical trial of VLS-101 is ongoing in patients with R/R lymphomas and leukemias. One particularly promising feature of VLS-101 is its potential use in Richter syndrome (RS), as this agent has been effective in eliminating xenografts derived from RS patients in mice.

\section{Conclusions}

ADCs exploit the antigen specificity of mAbs to selectively deliver highly potent cytotoxic payloads to tumors while sparing normal tissues. Lymphomas present attractive clinical scenarios in which to utilize ADCs, in part due to their relatively unique surface antigen repertoires. The anti-CD30 agent brentuximab vedotin has been approved for use in untreated and R/R HL, as well as in several R/R $\mathrm{T}$ cell lymphomas. More recently, the anti-CD79b agent PV was approved for use in R/R DLBCL, and key clinical trials are underway to study the incorporation of this agent into frontline multiagent treatment regimens for DLBCL. Substantial advances in mAb optimization and linkage chemistry have facilitated significant progress in $\mathrm{ADC}$ design, leading to a number of novel agents recently entering clinical trials. Preliminary data suggest that several of these novel ADCs may possess attractive therapeutic windows, and subsequent studies will shed light on how to pair these therapies with other agents possessing complementary targets, mechanisms of action, and AE profiles.

FINANCIAL DISCLOSURE: The authors have no significant financial interest in or other relationship with the manufacturer of any product or provider of any service mentioned in this article.

For full reference list, visit cancernetwork.com/Lymphoma_ADC

\section{Russler-Germain}

is a hematology/oncology fellow at the Washington University School of Medicine.

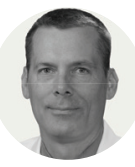

Tahl

is a professor in the Department of Medicine, Divisions of Hematology/Oncology, at the Washington University School of Medicine. 


\section{WHAT}

\section{MATTERS}

A diagnosis of prostate cancer was life-changing news for over 170,000 people per year
Cutting-edge treatments that are noninvasive so he can enjoy a life-changing trip around the world

\section{cancernetwork \\ home of the journal ONCOLOGY ${ }^{\circ}$}

Oncology and CancerNetwork lead the industry with the latest insights from key opinion leaders in oncology through articles, peer perspectives, and interactive content that translates into clinical application for today's practicing oncologist.

Timely. Practical. Relevant. 


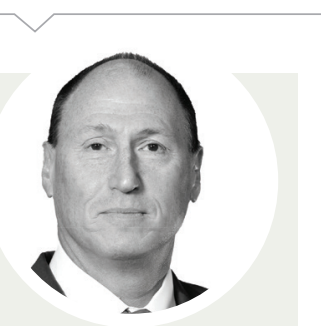

Lilenbaum is

director, Banner MD Anderson Cancer Center

\title{
Harnessing the Breakthroughs in Lung Cancer Treatment
}

\author{
"We still have some challenging issues."
}

Lung cancer incidence and mortality in the United States have been on the decline, due in large part to a sharp reduction in tobacco use. ${ }^{1}$ Results of a recent study have brought additional good news: For patients with non-small cell lung cancer (NSCLC), mortality is decreasing faster than incidence, ${ }^{2}$ suggesting that the recent rise in the use of targeted therapies is having a very significant impact on overall survival.

But work still needs to be done. While mutational targets have been identified, and newer therapeutics have been approved, optimizing that targeted therapy and sequencing every patient in order to treat them in accordance with current guidelines and algorithms remains a challenge.

ONCOLOG $Y^{\circledR}$ recently sat down with Rogerio C. Lilenbaum, MD, director of Banner MD Anderson Cancer Center, to discuss current and future trends in the care of patients with lung cancer, as well as his transition from clinician to administrator and the considerable set of challenges that role brings.

\section{A. Can we look forward to any trends or developments for patients with lung cancer?}

LILENBAUM: I think we still have some challenging issues. We've been on the edge of identifying an agent for KRAS-mutated tumors; KRAS happens to be the most common mutation in lung cancer. I think that's exciting. So far it hasn't been actionable in the sense that we have an agent to target that mutation. But now new agents are out there showing promising activity in $K R A S$-mutated tumors. And then in immunotherapy, other agents that are not checkpoint inhibitors have other targets within the activation of the immune system. I think those agents are promising, and then you can take that concept of immune manipulation and take it all the way to even chimeric antigen receptor T cells, which we now use for leukemias and lymphomas. There are some promising early data showing that this approach may have some efficacy in solid tumors as well.

Also, when we talk about targeted agents, an aspect of this conversation that has always interested me, and at times baffled me, is how some data are quickly adopted and applied by the majority of practicing oncologists, and then other data sets that [also] appear to show significant benefit are not as widely adopted. In stage 3 disease, for example, we have unequivocal data that one checkpoint in- hibitor, durvalumab (Imfinzi), at least as of now, improves outcomes. And yet, to the best of my knowledge, utilization of that strategy is not as high as it should be. Granted, the management of stage $2 \mathrm{~B}$ patients is complicated, and not everyone follows the [same] paradigm: In order [for a patient] to get to the durvalumab [treatment], you have to have received concomitant chemo and radiation, and not everybody does. [Yet,] it surprises me at times that powerful data like this are still not applied more widely, as they should be.

\section{You are a cochair for C.the 18th Annual Winter Lung Conference ${ }^{\circledR}$. What can we look forward to during that meeting, and what makes the meeting so beneficial for oncologists?}

LILENBAUM: I think one benefit of attending Winter Lung is the opportunity to hear about the most important advances in the field of lung cancer through the opinions of experts. You see the data and have an opportunity to understand the most impactful trials and studies that have been done in the recent past. And then on top of that, you have an opportunity to get editorial comments and personal opinions from national and international experts. 
That's a hallmark of Winter Lung and it has been for quite some time.

A second benefit is that we tend to give everybody an opportunity to present a case. We have case presentations on the agenda during the meeting. So, Winter Lung becomes a very real-world conference in the sense that you're discussing real patients in real time among experts from different disciplines. It's both an academic exercise in the sense that you hear the latest in the interpretation of the experts, and it's also a very clinical and practical conference in the sense that you get to apply that knowledge to patient care. And I think that balance has always characterized a Winter Lung conference.

\section{What are some of the Eclinical issues that will be discussed?}

LILENBAUM: One question I'd like to see discussed is whether we have optimized the use of targeted therapy. We now have a set number of actionable molecular alterations. It's not likely that we will see many new ones or new targets discovered in the near future. Are we testing everyone for all the actionable mutations? And are we treating those patients according to evidence-based algorithms and guidelines? This may seem very obvious, but it's far from obvious in clinical practice.

I think a significant percentage of patients with NSCLC and nonsquamous histology who have potentially actionable mutations don't always get treated as such. And I think that's an important issue.

\section{Q: Why is that? Why are we not optimizing the use of targeted therapy for these patients?}

LILENBAUM: First, I think, is that testing in this day and age is still somewhat erratic. It's not as pervasive or as generalized as it should be. Then, even within each categoryEGFR, ALK, ROS, RET, $B R A F$ - the information about how to best manage those patients is not always within the domain of the average oncologist. This was further complicated by the advent of immunotherapy.

A second issue is how we can identify patients who truly benefit from checkpoint inhibitors beyond just PD-L1. In some patients, the benefit of checkpoint inhibitors is nothing short of extraordinary. We've seen this from years of research. But in other patients, the benefit is fairly modest. Yes, PD-L1 is a marker that can help ascertain that magnitude of benefit, but it's far from perfect. And I think we need additional predictors or predictive factors to justify the cost and the toxicity of these agents.

Those are 2 very practical, broad issues in terms of [patient] management. I think we now are beginning to see the application of these 2 strategies to early-stage disease. That would be a third point. We now have the data for osimertinib in the adjuvant setting for EGFR-positive patients. That, to me, is a breakthrough, and it will change the way we do adjuvant treatment. And I think we're going to see similar data for other molecular alterations. More importantly, we will see data for immunotherapy in the early-stage settings. So I think, again, that how you apply the knowledge that we've acquired in late-stage disease to early-stage disease is among the most potentially impactful areas of research. When you [use immunotherapy] in early-stage disease, what you're doing is not only prolonging life, but increasing the cure rate. And that's what we want to see.

\section{A. When it comes to -immunotherapy, the} term "cure" comes up with other cancers, notably with melanoma, but not, it seems, with lung cancer. Why is that?

LILENBAUM: I have made that comment a few times. Number 1, I think we don't have as long a follow-up in patients with lung cancer as we have in patients with melanoma. Number 2, when you get to the bottom line, it doesn't really appear that immunotherapy in lung cancer has the same overall impact as it does in melanoma. So, I think it is harder to apply that concept of cure to advanced disease in lung cancer at this point.

\section{You're originally from Brazil. How did you end up here in the United States?}

LILENBAUM: I came purely for professional reasons. After finishing school and beginning my training, I realized I wanted to become a medical oncologist. At that time, though, Brazil had very few options for oncology training, so I came to the United States.

I enjoyed not only the practice of medicine in the United States, but the academic com- ponent and specifically the opportunity to be involved in clinical research. By the time I was finishing my fellowship, I really couldn't see myself in any other place, and I was very fortunate to have outstanding mentors who opened a lot of doors and opportunities for me. So, I had an early entry into the world of lung cancer and lung cancer investigation and research.

\section{. What was going on -at that point that attracted you to the field?}

LILENBAUM: This was the mid1990s. We had a huge influx of new chemotherapy drugs in lung cancer, and this was a disease that up until the early to mid-1990s had essentially 1 or 2 regimens that had shown some efficacy, and, by the way, horrendous toxicity. The new drugs, at the time, seemed to provide greater benefit and much less toxicity. So, the benefit ratio for treating advanced patients at the time improved significantly, and there was a lot of conversation about how to utilize those new drugs. These drugs-vinorelbine and gemcitabine, just to name 2- came into life in the mid1990s and dominated the conversation about treating advanced NSCLC, including mega-trials about what was the optimal regimen: Is there a better combination of drugs? Do you really need 2 drugs, or is 1 drug sufficient in some patients? Also: Do you really need a platinum agent? Can you do a nonplatinum combination? How do you treat elderly patients or patients with a performance status of 2? Those were questions that dominated the conversa- 
tion, I would say from 1995 to 2005 , when we started to steer more in the direction of targeted therapy, and that's where I cut my teeth in lung cancer.

\section{Along your career 1 - path, you transitioned into a more administrative position. What took you there?}

LILENBAUM: I felt that after about 12 or 15 years of a very active clinical practice, plus a very active clinical research career, I wanted to have a different impact on how cancer care is delivered-to take this experience into organizations that were creating comprehensive and integrated cancer care delivery systems. That was my impetus. It has been, to some extent, a long journey. It is, in many ways, much more challenging than clinical care or clinical research, but no less important.

\section{Can you talk about Isome of those challenges?}

LILENBAUM: Well, first, physicians in my generation were not trained in, and did not necessarily graduate from fellowship with, organization skills or leadership skills. If anything, we were trained to acquire a certain body of knowledge and make decisions independently on behalf of the individual who is in front of you and happens to be a patient at the time. Then [as an administrator] you take that experience, or even the experience of research, which applies to a larger population, but [these people] are mostly invisible to you. Then you think about, How do we do this at a larger scale, in a way that is more structural and less individual, that is more foundational? Above all, [how do we do it] in a way that sets a culture for high-quality service to patients? That's why I decided to do this. And I had the opportunity when I was recruited to be the chief medical officer for the Yale Cancer Center and the Yale Cancer Hospital, and I worked with some of the best people in the country in every aspect of cancer care and was exposed to a tremendous amount of information and knowledge that I did not acquire formally. I don't have an MBA, for example, as some [colleagues of mine] do. I also had the opportunity-or the responsibility, I guess-to implement changes that would change how we delivered care across, in that case, the whole state of Connecticut. I was exposed to terrific leaders, people who had already successfully done that. So, it was a terrific experience for me, and-just to "close the loop" here-I felt, when I was approached to consider this position at Banner MD Anderson, that I was prepared to lead an entire cancer program in that direction. I wanted to take on a program that was poised to take a qualitative step toward excellence, and patient centeredness, and an integrated delivery system, and that's why I moved to Phoenix, Arizona.

\section{A. What exactly do you - mean by patient- centered and integrated delivery?}

LILENBAUM: When people talk about "patient experience," the automatic reflex is to think about welcoming patients or being nice to patients. That's really only the floor, the minimum required for anyone to interact with patients at any point in time. Patience experience is really the totality of the experience of these individuals, like you and me, or loved ones, who, at that moment, are afflicted with a very serious illness and are very vulnerable and dependent on people who can provide knowledge and support to guide them through that process. So, it's about the clinical piece and it's about the operational piece, which is something we focus on quite a bit. It's also about the financial piece and the cultural piece. [Unfortunately,] we don't think of access as being a patient-centered exercise, but instead as a business opportunity. If you see patients quickly, they'll come to you, as opposed to having to wait a week or 2. Yet at the end of the day, it really is unconscionable to keep someone who was just given a diagnosis of cancer waiting for weeks to see a physician, just to obtain a basic understanding of what their illness is. And that's a humanistic component. It's not about market share. It's just the way we all [should] expect to be treated under these circumstances.

Another piece that we physicians often are disconnected from is, I think, the financial piece- having an organized financial picture of what going through cancer treatment means for the patient and their family's well-being. Having an orderly way to manage bills, and having a way to pay for those bills, is all related to patient-centered care, and it's a huge onus that we impose on our patients and their families. We don't really have very a good structure to support them through that process.

Now in terms of integrated care, what we talk about here is a signature care for Banner MD Anderson. It's that sense that if you have, say, breast cancer, you will enter the Banner MD Anderson facility and you will have the same experience as another patient with breast cancer who enters a different MD Anderson facility in a different state. It's what characterizes what we, as a cancer program, have to offer our patients in terms of clinical excellence, operational excellence, and in strong and compassionate support throughout that experience. And to create that alignment, to create that sense that it's all one package, is a pretty challenging goal from an administrative and financial perspective

\section{FINANCIAL DISCLOSURE: The}

authors have no significant financial interest in or other relationship with the manufacturer of any product or provider of any service mentioned in this article..

\section{REFERENCES}

1. Cancer stat facts: lung and bronchus cancer. National Cancer Institute: Surveillance, Epidemiology, and End Results Program. Accessed November 9, 2020. https://seer.cancer.gov/ statfacts/html/lungb.html

2. Howlader N, Forjaz G, Mooradian MJ, et al. The effect of advances in lung-cancer treatment on population mortality. $N$ Engl J Med. 2020;383(7):640-649. doi:10.1056/NEJMoa1916623 


\section{FDA Approves FoundationOne Liquid CDx as Companion Diagnostic for Olaparib}

The FDA has approved the FoundationOne Liquid CDx to be used as a companion diagnostic for olaparib (Lynparza), according to Foundation Medicine, the developer of the companion diagnostic.

The FoundationOne Liquid CDx uses a blood-based biopsy to identify BRCA1, $B R C A 2$, and/or ATM alterations in patients with metastatic castration-resistant prostate cancer who may be appropriate for treatment with olaparib. The comprehensive genomic profiling test is now approved as a companion diagnostic for 7 targeted therapies across 4 tumor types.

The FoundationOne Liquid CDx is a qualitative next-generation sequencingbased in vitro diagnostic test, available only by prescription, that uses targeted high throughput hybridization-based capture technology to analyze 324 genes. It utilizes circulating cell-free DNA isolated from plasma that is derived from anticoagulated peripheral whole blood of advanced cancer patients. Additional genomic findings may be reported by the test; however, these are not prescriptive or conclusive for labeled use of any specific therapeutic product.

Moreover, use of the test does not guarantee that a patient will be matched to a treatment. A negative result does not rule out the presence of an alteration. Patients who are negative for companion diagnostic mutations should be reflexed to tumor tissue testing, and their mutation status should be confirmed using an FDA-approved tumor tissue test, if feasible.

The FoundationOne Liquid CDx was approved by the FDA in August to report genomic alteration results for patients with any solid tumor. Notably, Foundation Medicine's tissue-based comprehensive genomic profiling test, FoundationOne CDx, was also approved as a companion diagnostic for olaparib in May 2020.

\section{REFERENCE}

Foundation Medicine expands indication for FoundationOne Liquid CDx to be used as a companion diagnostic for Lynparza. News release. Foundation Medicine; November 9, 2020. Accessed November 9, 2020. https://www.foundationmedicine.com/ press-releases/3ace3473-1335-43bd-94542388c5549bf8

To read the full article visit: cancernetwork.com/Olaparib_diagnostic

\section{Enzalutamide Induces Added Benefit for Patients With Castration-Resistant Prostate Cancer}

Researchers have found an added benefit in terms of mortality due to the longer overall survival with the drug enzalutamide (Xtandi) to treat adult patients with high-risk nonmetastatic castration-resistant prostate cancer, according to a reassessment by the German Institute for Quality and Efficiency in Health Care.

The drug had previously been examined twice, before in 2018 and 2019, to see if there was an advantage in comparison with the appropriate comparator therapy. Both the first and second data cut-offs for the PROSPER study found no added benefit for the drug to treat highrisk nonmetastatic castration-resistant prostate cancer.

In regard to morbidity and health-related quality of life, no new findings existed when compared with the first assessment of the drug. As a result, no added benefit was determined for these outcome categories.

Where that differed was in terms of mortality for the third data cut-off when compared with the first assessment. The data indicated that patients in the enzalutamide arm survived considerably longer than patients in the comparator arm.

The researchers explained that the results were mixed in terms of the drug's adverse effects (AEs). For renal and urinary disorders, there were signs of a major AE advantage for patients, although it is unclear whether this reduced AE profile was a result of treatment or symptoms of the disease. More disadvantages were found for enzalutamide when compared with watchful waiting while maintaining ongoing conventional androgen deprivation therapy.

Regardless, the disadvantages did not put the advantages into question. The overall assessment found an added benefit for enzalutamide compared with the appropriate comparator therapy.

\section{REFERENCE}

Castration-resistant prostate cancer at high risk of metastasis: enzalutamide has added benefit. News release. American Association for the Advancement of Science; August 19, 2020. Accessed November 14, 2020. https://www. eurekalert.org/pub_releases/2020-08/ifqacpc081920.php.

\section{To read the full article visit:} cancernetwork.com/enzalutamide_crpc

\section{Lenvatinib in Combination With Pembrolizumab or Everolimus Shows Promise for Advanced RCC}

New investigational data from the pivotal phase 3 KEYNOTE-581/CLEAR trial (Study 307) demonstrated that the combination of pembrolizumab (Keytruda) and lenvatinib (Lenvima) in patients with advanced renal cell carcinoma (RCC) met the study's primary end point of progression-free survival (PFS) and its key secondary end points of overall survival (OS) and objective response rate (ORR).

The results, announced by Merck, demonstrated statistically significant and clinically meaningful improvements in PFS, OS, and ORR vs lenvatinib combined with sunitinib (Sutent) in the intention-to-treat (ITT) study population. Additionally, lenvatinib plus everolimus met the trial's primary end point of PFS and a key secondary end point of ORR, demonstrating a statistically significant and clinically meaningful improvements in both vs the sunitinib combination in the ITT study population.

The safety profiles of both pembrolizumab plus lenvatinib and lenvatinib 
plus everolimus were found to be consistent with previously reported studies.

The multicenter, randomized, open-label, phase 3 trial is evaluating lenvatinib in combination with pembrolizumab or in combination with everolimus vs sunitinib for the first-line treatment of patients with advanced RCC. The primary end point is PFS by independent review per RECIST 1.1 criteria and key secondary end points include OS, ORR, and safety.

The study enrolled approximately 1050 patients who were randomized to 1 of 3 treatment arms to receive either:

- $18 \mathrm{mg}$ of lenvatinib orally once daily in combination with $5 \mathrm{mg}$ orally once daily of everolimus; or

- $20 \mathrm{mg}$ of lenvatinib orally once daily in combination with $200 \mathrm{mg}$ of pembrolizumab intravenously every 3 weeks; or

- 50 mg of sunitinib orally once daily for 4 weeks on treatment, followed by 2 weeks off treatment.

The ITT population included patients across all Memorial Sloan Kettering Cancer Center risk groups, including favorable, intermediate, and poor.

Merck and Eisai indicated that they will discuss these data with regulatory authorities worldwide, with the intent to submit marketing authorization applications based on these results. In addition, they will be submitting the results to be presented at an upcoming medical meeting.

Of note, the companies are continuing to study the pembrolizumab plus lenvatinib combination through the LEAP (LEnvatinib And Pembrolizumab) clinical program across 19 trials in 13 different tumor types, including endometrial carcinoma, hepatocellular carcinoma, melanoma, non-small cell lung cancer, RCC, squamous cell carcinoma of the head and neck, urothelial cancer, biliary tract cancer, colorectal cancer, gastric cancer, glioblastoma, ovarian cancer, and triple-negative breast cancer.

\section{REFERENCE}

Keytruda (pembrolizumab) plus Lenvima (lenvatinib) demonstrated statistically significant improvement in progression-free survival (PFS), overall survival (OS) and objective response rate (ORR) versus sunitinib as first-line treatment for patients. News release. Merck; November 10, 2020. Accessed November 14, 2020. https://www.merck.com/news/ keytruda-pembrolizumab-plus-lenvimalenvatinib-demonstrated-statistically-significantimprovement-in-progression-free-survival-pfsoverall-survival-os-and-objective-response-rate/

To read the full article visit: cancernetwork.com/lenvatinib_rcc

\section{Tremelimumab, Durvalumab Combo Shows Promise for Certain Patients with Localized Bladder Cancer}

Phase 1 clinical trial results published in Nature Medicine demonstrated that neoadjuvant combination treatment with tremelimumab and durvalumab (Imfinzi) was well tolerated and showed early signs of activity in certain patients with localized bladder cancer who are not eligible for cisplatin-based chemotherapy. ${ }^{1}$

Patients included in the study had tumors with high-risk features that are associated with unfavorable outcomes, defined by bulky tumors, variant histology, lymphovascular invasion, hydronephrosis, and/or high-grade upper tract disease.

Between April 2017 and December 2018, researchers enrolled 28 patients with high-risk, cisplatin-ineligible, localized muscle-invasive urothelial carcinoma in the first cohort of the study. Participants had baseline transurethral resection of bladder tumor and were then treated with a combination of durvalum$\mathrm{ab}$ at a dose of $1500 \mathrm{mg} / \mathrm{kg}$ and tremelimumab at a dose of $75 \mathrm{mg} / \mathrm{kg}$ every 4 weeks for a total of 2 combination doses before cystectomy.

No deaths related to therapy occurred. However, the majority of patients experienced an immune-related adverse event (irAE) of any grade. The most common irAE was grade 1 or 2 rash and asymptomatic increase in amylase; each oc- curred in $29 \%$ of patients. Additionally, the study did not exceed its safety or futility rules, with a total of 6 patients $(21 \%)$ experiencing grade 3 or higher irAEs.

Of the 28 patients enrolled in the trial, 24 completed cystectomy per protocol, with $9(37.5 \%)$ achieving a pathologic complete response (pCR). Moreover, in 12 patients with particularly large tumors (stage T3-T4), the pCR rate was $42 \%$, with half of those patients seeing their tumor size reduced to stage T1 or less. $^{2}$

Importantly, median overall survival (OS) has not yet been reached, and 24 patients were still alive at 1 year. Additionally, $82.8 \%$ of patients who had surgery were free of disease recurrence at 1 year.

The researchers also collected pre- and posttreatment blood and tissue samples from patients to study biomarkers associated with response, identifying higher-density tertiary lymphoid structures (TLS) in pretreatment tumor samples from patients who responded well to combination therapy compared with those who did not respond. Notably, a higher density of TLS correlated with longer OS and relapse-free survival.

Although these results still need to be confirmed in larger studies, investigators indicated that the findings suggest that TLS may act as an effective predictive biomarker for patients who will respond to checkpoint blockade.

\section{REFERENCES}

1. Gao J, Navai N, Alhalabi O, et al. Neoadjuvant PD-L1 plus CTLA-4 blockade in patients with cisplatin-ineligible operable high-risk urothelial carcinoma. Nat Med. Published online October 12, 2020. doi:10.1038/s41591-020-1086-y

2. Dual checkpoint blockade promising as pre-surgical approach for certain patients with localized bladder cancer. News release. The University of Texas MD Anderson Cancer Center; October 12, 2020. Accessed November 14, 2020. https://www.mdanderson.org/newsroom/dualcheckpoint-blockade-promising-as-pre-surgicalapproach-for-certain-patients-with-localizedbladder-cancer.h00-159385890.html

To read the full article visit: cancernetwork.com/tremelimumab_bc 


\section{IN THE TREATMENT OF RELAPSED REFRACTORY MULTIPLE MYELOMA \\ IN COMBINATION WITH POMALIDOMIDE AND DEXAMETHASONE (Pd)}

\section{ACHIEVE GREATER OUTCOMES FOR YOUR PATIENTS}

SARCLISA is an anti-CD38 therapy proven to deliver superior PFS (median PFS of 11.53 months with SARCLISA + Pd vs 6.47 months with Pd alone, $\mathrm{HR}=0.596,95 \% \mathrm{Cl}: 0.44,0.81, P=0.0010$ ). SARCLISA also demonstrated a significant increase in ORR $160.4 \%$ with SARCLISA + Pd [95\% Cl: $52.2 \%, 68.2 \%$ ] vs $35.3 \%$ with Pd alone [95\% Cl: $27.8 \%, 43.4 \%], P<0.0001)^{1 *}$

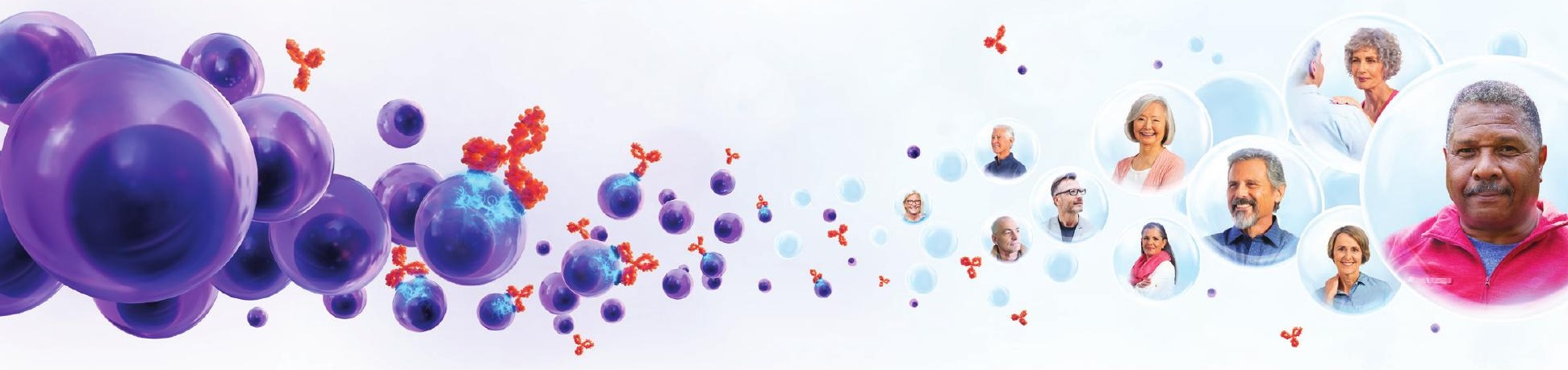

NCCN Clinical Practice Guidelines in Oncology (NCCN Guidelines ${ }^{\ominus}$ ) Preferred Category 1 recommendation for isatuximab-irfc (SARCLISA)

Isatuximab-irfc (SARCLISA), in combination with pomalidomide and dexamethasone, is a Preferred Category 1 option for previously treated multiple myeloma by the National Comprehensive Cancer Network ${ }^{\circledast}\left(\mathrm{NCCN}^{\circledast}\right)^{2}{ }^{2}$

NCCN makes no warranties of any kind whatsoever regarding their content, use or application and disclaims any responsibility for their application or use in any way.

*ORR included SCR, CR, VGPR, and PR. SCR, CR, VGPR, and PR were evaluated by an IRC using the IMWG response criteria.'

$C R=$ complete response; IMWG=International Myeloma Working Group; IRC=independent response committee; $m A b=$ monoclonal antibody; NCCN=National Comprehensive Cancer Network; ORR=overall response rate; PFS=progression-free survival; PR=partial response; $\mathrm{s} C R=$ stringent complete response; $\mathrm{VGPR}=$ very good partial response.

\section{Indication}

SARCLISA (isatuximab-irfc) is indicated, in combination with pomalidomide and dexamethasone, for the treatment of adult patients with multiple myeloma who have received at least two prior therapies including lenalidomide and a proteasome inhibitor.

\section{Important Safety Information}

\section{CONTRAINDICATIONS}

SARCLISA is contraindicated in patients with severe hypersensitivity to isatuximab-irfc or to any of its excipients.

\section{WARNINGS AND PRECAUTIONS}

\section{Infusion-Related Reactions}

Infusion-related reactions (IRRs) have been observed in $39 \%$ of patients treated with SARCLISA. All IRRs started during the first SARCLISA infusion and resolved on the same day in $98 \%$ of the cases. The most common symptoms of an IRR included dyspnea, cough, chills, and nausea. The most common severe signs and symptoms included hypertension and dyspnea.

Please see Important Safety Information throughout, and accompanying brief summary of full Prescribing Information. 


\title{
Choose SARCLISA + Pd to Offer Improved Outcomes to More Patients vs Pd Alone
}

\author{
Studied in the phase 3 ICARIA-MM trial, which included \\ patients with poor prognostic factors ${ }^{1}$
}

\author{
Based on the ICARIA-MM trial, SARCLISA + Pd is a treatment choice \\ for patients with relapsed refractory multiple myeloma
}

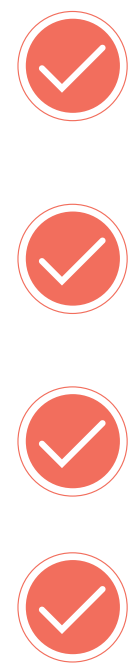

\author{
Who have received at least 2 prior therapies, \\ including lenalidomide and a PI
}

Who may have renal impairment (creatinine clearance $<60 \mathrm{~mL} / \mathrm{min} / 1.73 \mathrm{~m}^{2}$ ), high cytogenetic risk, or a history of COPD or asthma

\section{Who may have poor performance status} or are $\geq 75$ years of age

\author{
Who are refractory to lenalidomide, a PI, \\ or both
}

\begin{abstract}
STUDY DESIGN: ICARIA-MM (NCTO2990338), a multicenter, open-label, randomized, phase 3 study, evaluated the efficacy and safety of SARCLISA in 307 patients with relapsed refractory multiple myeloma who had received at least 2 prior therapies, including lenalidomide and a PI. Patients received either SARCLISA $10 \mathrm{mg} / \mathrm{kg}$ administered as an IV infusion in combination with Pd ( $\mathrm{n}=154)$ or Pd alone ( $\mathrm{n}=153)$, administered in 28-day cycles until disease progression or unacceptable toxicity. SARCLISA was given weekly in the first cycle and every 2 weeks thereafter. Pomalidomide $4 \mathrm{mg}$ was taken orally once daily from day 1 to day 21 of each 28-day cycle. Low-dose dexamethasone (orally or IV) $40 \mathrm{mg}$ ( $20 \mathrm{mg}$ for patients $\geq 75$ years of age) was given on days 1, 8, 15, and 22 for each 28 -day cycle. PFS was the primary endpoint; ORR and OS were key secondary endpoints. PFS results were assessed by an IRC, based on central laboratory data for M-protein, and central radiologic imaging review using the IMWG criteria. Median follow-up was 11.6 months.'
\end{abstract}

PATIENT CHARACTERISTICS: The median patient age was 67 years (range, 36 to 86 ), and $20 \%$ of patients were $\geq 75$ years of age. Ten percent of patients entered the study with a history of COPD or asthma. The proportion of patients with renal impairment (creatinine clearance $<60 \mathrm{~mL} / \mathrm{min} / 1.73 \mathrm{~m}^{2}$ ) was $34 \%$. The ISS stage at study entry was I in $37 \%$, II in $36 \%$, and III in $25 \%$ of patients. Overall, $20 \%$ of patients had high-risk chromosomal abnormalities at study entry: del(17p), $t(4 ; 14)$, and $t(14 ; 16)$ were present in $12 \%, 8 \%$, and $2 \%$ of patients, respectively. The median number of prior lines of therapy was 3 (range, 2 to 11). All patients received a prior PI, all patients received prior lenalidomide, and $56 \%$ of patients received prior stem cell transplantation; the majority of patients (93\%) were refractory to lenalidomide, $76 \%$ to a Pl, and $73 \%$ to both an immunomodulator and a PI.

COPD=chronic obstructive pulmonary disease; ISS=International Staging System; IV=intravenous; OS=overall survival; $\mathrm{Pl}=$ proteasome inhibitor .

\section{Important Safety Information (cont'd)}

\section{Infusion-Related Reactions (cont'd)}

To decrease the risk and severity of IRRs, premedicate patients prior to SARCLISA infusion with acetaminophen, $\mathrm{H}_{2}$ antagonists, diphenhydramine or equivalent, and dexamethasone. Monitor vital signs frequently during the entire SARCLISA infusion. For patients with grade 1 or 2 reactions, interrupt SARCLISA infusion and provide appropriate medical support. If symptoms improve, restart SARCLISA infusion at half of the initial rate, with supportive care as needed, and closely monitor patients. If symptoms do not recur after 30 minutes, the infusion rate may be increased to the initial rate, and then increased incrementally. In case symptoms do not improve or recur after interruption, permanently discontinue SARCLISA and institute appropriate management. Permanently discontinue SARCLISA if a grade 3 or higher IRR occurs and institute appropriate emergency medical management. 


\section{SARCLISA + Pd Extended Median PFS to 1 Year}

\section{Superior PFS with SARCLISA + Pd vs Pd alone}

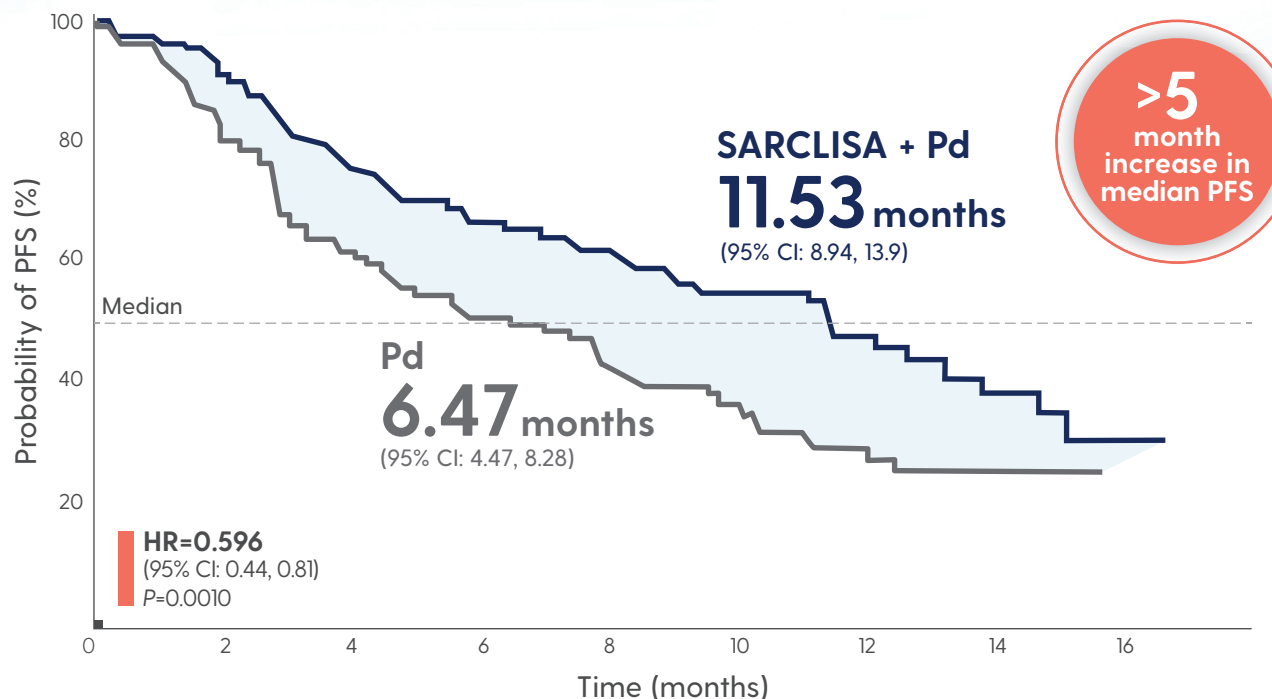

Patients at risk

\begin{tabular}{rllllllllll} 
SARCLISA + Pd & 154 & 129 & 106 & 89 & 81 & 52 & 30 & 14 & 1 \\
\cline { 2 - 11 } & 153 & 105 & 80 & 63 & 51 & 33 & 17 & 5 & 0
\end{tabular}

The median duration of treatment was 41 weeks with SARCLISA + Pd vs 24 weeks with Pd.'

At a median follow-up time of 11.6 months, 43 patients (27.9\%) receiving SARCLISA + Pd and 56 patients (36.6\%) receiving Pd had died. Median OS was not reached for either treatment group at interim analysis. The OS results at interim analysis did not reach statistical significance.'

\section{SARCLISA + Pd showed a significant increase in ORR ${ }^{1 *}$}

\begin{tabular}{ccc} 
SARCLISA + Pd $(n=154)$ & $P d(n=153)$ \\
\hline $60.4 \%$ ORR & $P<0.0001$ & $35.3 \%$ ORR \\
\hline $31.8 \% \geq V G P R$ & $\sim 4 \times$ increase & $\mathbf{8 . 5} \% \geq V G P R$ \\
\hline 35 days & $\begin{array}{c}\text { Median time to first response } \\
\text { among responders }\end{array}$ & $\mathbf{5 8}$ days
\end{tabular}

*ORR included sCR, CR, VGPR, and PR. ORR: SARCLISA + Pd (95\% Cl: 52.2\%, 68.2\%), Pd (95\% Cl: 27.8\%, 43.4\%).

\section{Important Safety Information (cont'd)}

\section{Neutropenia}

SARCLISA may cause neutropenia. Neutropenia (reported as laboratory abnormality) occurred in $96 \%$ of patients and grade 3-4 neutropenia occurred in $85 \%$ of patients treated with SARCLISA, pomalidomide, and dexamethasone (Isa-Pd). Febrile neutropenia occurred in $12 \%$ of patients and neutropenic infections, defined as infection with concurrent grade $\geq 3$ neutropenia, occurred in $25 \%$ of patients treated with Isa-Pd. The most frequent neutropenic infections included those of upper respiratory tract $(10 \%)$, lower respiratory tract $(9 \%)$, and urinary tract $(3 \%)$.

Please see Important Safety Information throughout, and accompanying brief summary of full Prescribing Information. 


\section{Important Safety Information (cont'd)}

\section{Neutropenia (cont'd)}

Monitor complete blood cell counts periodically during treatment. Consider the use of antibiotics and antiviral prophylaxis during treatment. Monitor patients with neutropenia for signs of infection. In case of grade 4 neutropenia, delay SARCLISA dose until neutrophil count recovery to at least $1.0 \times 10^{\circ} / \mathrm{L}$, and provide supportive care with growth factors, according to institutional guidelines. No dose reductions of SARCLISA are recommended.

\section{Second Primary Malignancies}

Second primary malignancies were reported in $3.9 \%$ of patients in the SARCLISA, pomalidomide, and dexamethasone (Isa-Pd) arm and in $0.7 \%$ of patients in the pomalidomide and dexamethasone (Pd) arm, and consisted of skin squamous cell carcinoma (2.6\% of patients in the Isa-Pd arm and in $0.7 \%$ of patients in the Pd arm), breast angiosarcoma ( $0.7 \%$ of patients in the Isa-Pd arm), and myelodysplastic syndrome $(0.7 \%$ of patients in the Isa-Pd arm). With the exception of the patient with myelodysplastic syndrome, patients were able to continue SARCLISA treatment. Monitor patients for the development of second primary malignancies.

\section{Laboratory Test Interference}

\section{Interference with Serological Testing (Indirect Antiglobulin Test)}

SARCLISA binds to CD38 on red blood cells (RBCs) and may result in a false positive indirect antiglobulin test (indirect Coombs test). In ICARIA-multiple myeloma (MM), the indirect antiglobulin test was positive during SARCLISA treatment in $67.7 \%$ of the tested patients. In patients with a positive indirect antiglobulin test, blood transfusions were administered without evidence of hemolysis. ABO/RhD typing was not affected by SARCLISA treatment. Before the first SARCLISA infusion, conduct blood type and screen tests on SARCLISA-treated patients. Consider phenotyping prior to starting SARCLISA treatment. If treatment with SARCLISA has already started, inform the blood bank that the patient is receiving SARCLISA and SARCLISA interference with blood compatibility testing can be resolved using dithiothreitol-treated RBCs. If an emergency transfusion is required, non-cross-matched $A B O / R h D-$ compatible RBCs can be given as per local blood bank practices.

\section{Interference with Serum Protein Electrophoresis and Immunofixation Tests}

SARCLISA is an IgG kappa monoclonal antibody that can be incidentally detected on both serum protein electrophoresis and immunofixation assays used for the clinical monitoring of endogenous M-protein. This interference can impact the accuracy of the determination of complete response in some patients with lgG kappa myeloma protein.

\section{Embryo-Fetal Toxicity}

Based on the mechanism of action, SARCLISA can cause fetal harm when administered to a pregnant woman. SARCLISA may cause fetal immune cell depletion and decreased bone density. Advise pregnant women of the potential risk to a fetus. Advise females with reproductive potential to use an effective method of contraception during treatment with SARCLISA and for at least 5 months after the last dose. The combination of SARCLISA with pomalidomide is contraindicated in pregnant women because pomalidomide may cause birth defects and death of the unborn child. Refer to the pomalidomide prescribing information on use during pregnancy.

\section{ADVERSE REACTIONS}

The most common adverse reactions ( $\geq 20 \%$ ) were neutropenia (laboratory abnormality, $96 \%$ Isa-Pd vs $92 \% \mathrm{Pd}$ ), infusion-related reactions ( $38 \% \mathrm{Isa}-\mathrm{Pd}$ vs $0 \% \mathrm{Pd}$ ), pneumonia ( $31 \% \mathrm{Isa}-\mathrm{Pd}$ vs $23 \% \mathrm{Pd}$ ), upper respiratory tract infection ( $57 \%$ Isa-Pd vs $42 \%$ Pd), and diarrhea ( $26 \%$ with Isa-Pd vs $19 \% \mathrm{Pd}$ ). Serious adverse reactions occurred in $62 \%$ of patients receiving SARCLISA. Serious adverse reactions in $>5 \%$ of patients who received Isa-Pd included pneumonia $(26 \%)$, upper respiratory tract infections (7\%), and febrile neutropenia $(7 \%)$. Fatal adverse reactions occurred in $11 \%$ of patients (those that occurred in more than $1 \%$ of patients were pneumonia and other infections [3\%]).

\section{USE IN SPECIAL POPULATIONS}

Because of the potential for serious adverse reactions in the breastfed child from isatuximab-irfc administered in combination with Pd, advise lactating women not to breastfeed during treatment with SARCLISA.

\section{Please see accompanying brief summary of full Prescribing Information.}

References: 1. SARCLISA [prescribing information]. Bridgewater, NJ: sanofi-aventis U.S. LLC. 2. Referenced with permission from the NCCN Clinical Practice Guidelines in Oncology (NCCN Guidelines ${ }^{\circledR}$ ) for Multiple Myeloma V.2.2021. @ National Comprehensive Cancer Network, Inc. 2020. All rights reserved. Accessed September 9, 2020. To view the most recent and complete version of the guideline, go online to NCCN.org. 
SARCLISA ${ }^{\circledR}$

(isatuximab-irfc) injection, for intravenous use Brief Summary of Prescribing Information 1 INDICATIONS AND USAGE

SARCLISA is indicated, in combination with pomalidomide and dexamethasone, for the treatment of adult patients with multiple myeloma who have received at least two prior therapies including lenalidomide and a proteasome inhibitor.

\section{DOSAGE AND ADMINISTRATION}

\subsection{Recommended Dosage}

- Administer pre-infusion medications [see Dosage and

Administration (2.2)]

- SARCLISA should be administered by a healthcare professional, with immediate access to emergency equipment and appropriate medical support to manage infusion-related reactions if they occur [see Warnings and Precautions (5.1)].

The recommended dose of SARCLISA is $10 \mathrm{mg} / \mathrm{kg}$ actual body weight administered as an intravenous infusion in combination with pomalidomide and dexamethasone according to the schedule in Table 1 [see Clinical Studies (14) in the full prescribing information.

Table 1: SARCLISA Dosing Schedule in Combination with Pomalidomide and Dexamethasone

\begin{tabular}{|l|l|}
\hline Cycle & Dosing schedule \\
\hline Cycle 1 & Days 1, 8, 15, and 22 (weekly) \\
\hline Cycle 2 and beyond & Days 1, 15 (every 2 weeks) \\
\hline
\end{tabular}

Each treatment cycle consists of a 28-day period. Treatment is repeated until disease progression or unacceptable toxicity. SARCLISA is used in combination with pomalidomide and dexamethasone.

Missed SARCLISA Doses

If a planned dose of SARCLISA is missed, administer the dose as soon as possible and adjust the treatment schedule accordingly, maintaining the treatment interval.

\subsection{Recommended Premedications}

Administer the following premedications prior to SARCLISA infusion to reduce the risk and severity of infusion-related reactions [see Warnings and Precautions (5.1)].

- Dexamethasone $40 \mathrm{mg}$ orally or intravenously (or $20 \mathrm{mg}$

orally or intravenously for patients $\geq 75$ years of age)

- Acetaminophen $650 \mathrm{mg}$ to $1000 \mathrm{mg}$ orally (or equivalent)

- H2 antagonists.

- Diphenhydramine $25 \mathrm{mg}$ to $50 \mathrm{mg}$ orally or intravenously

(or equivalent). The intravenous route is preferred for at least the first 4 infusions.

The above recommended dose of dexamethasone (orally or intravenously) corresponds to the total dose to be administered only once before infusion as part of the premedication and of the backbone treatment, before SARCLISA and pomalidomide administration.

Administer the recommended premedication agents 15 to 60 minutes prior to starting a SARCLISA infusion.

\subsection{Dose Modifications}

No dose reduction of SARCLISA is recommended. Dose delay may be required to allow recovery of blood counts in the event of hematological toxicity [see Warnings and Precautions $(5.2,5.4)$ ]. For information concerning drugs given in combination with SARCLISA, see manufacturer's prescribing information For other medicinal products that are administered with

SARCLISA, refer to the respective current prescribing information

\subsection{Preparation}

Prepare the solution for infusion using aseptic technique as follows: Calculate the dose $(\mathrm{mg})$ of required SARCLISA based on actual patient weight (measured prior to each cycle to have the administered dose adjusted accordingly) [see Dosage and Administration (2.1)]. More than one SARCLISA vial may be necessary to obtain the required dose for the patient.

- Parenteral drug products should be inspected visually for particulate matter and discoloration prior to administration, whenever solution and container permit.

- Remove the volume of diluent from the $250 \mathrm{~mL}$ Sodium Chloride Injection, USP, or 5\% Dextrose Injection, USP diluent bag that is equal to the required volume of SARCLISA injection.

- Withdraw the necessary volume of SARCLISA injection and dilute by adding to the infusion bag of $0.9 \%$ Sodium Chloride Injection, USP or $5 \%$ Dextrose Injection, USP to achieve the appropriate SARCLISA concentration for infusion.

- The infusion bag must be made of polyolefins (PO), polyethylene (PE), polypropylene (PP), polyvinyl chloride (PVC) with di-(2-ethylhexyl) phthalate (DEHP) or ethyl viny acetate (EVA).

- Gently homogenize the diluted solution by inverting the bag. Do not shake.

\subsection{Administration}

- Administer the infusion solution by intravenous infusion using an intravenous tubing infusion set (in PE, PVC with or without DEHP, polybutadiene [PBD], or polyurethane [PU]) with a 0.22 micron in-line filter (polyethersulfone [PES], polysulfone, or nylon).

- The infusion solution should be administered for a period of time that will depend on the infusion rate (see Table 2). Use prepared SARCLISA infusion solution within 48 hours when stored refrigerated at $2^{\circ} \mathrm{C}-8^{\circ} \mathrm{C}$, followed by 8 hours (including the infusion time) at room temperature.

- Do not administer SARCLISA infusion solution concomitantly in the same intravenous line with other agents. nfusion Rates

Following dilution, administer the SARCLISA infusion solution intravenously at the infusion rates presented in Table 2. Incremental escalation of the infusion rate should be considered only in the absence of infusion-related reactions [see Warnings and Precautions (5.1) and Adverse Reactions (6.1)].

Table 2: Infusion Rates of SARCLISA Administration

\begin{tabular}{|l|c|c|c|c|c|}
\hline & $\begin{array}{c}\text { Dilution } \\
\text { Volume }\end{array}$ & $\begin{array}{c}\text { Initial } \\
\text { Rate }\end{array}$ & $\begin{array}{c}\text { Absence of } \\
\text { Infusion- } \\
\text { Related } \\
\text { Reaction }\end{array}$ & $\begin{array}{c}\text { Rate } \\
\text { Increment }\end{array}$ & $\begin{array}{c}\text { Maximum } \\
\text { Rate }\end{array}$ \\
\hline $\begin{array}{l}\text { First } \\
\text { infusion }\end{array}$ & $250 \mathrm{~mL}$ & $\begin{array}{c}25 \mathrm{~mL} / \\
\text { hour }\end{array}$ & $\begin{array}{c}\text { For } 60 \\
\text { minutes }\end{array}$ & $\begin{array}{c}25 \mathrm{~mL} / \text { hour } \\
\text { every } 30 \\
\text { minutes }\end{array}$ & $\begin{array}{c}150 \mathrm{~mL} / \\
\text { hour }\end{array}$ \\
\hline $\begin{array}{l}\text { Second } \\
\text { infusion }\end{array}$ & $250 \mathrm{~mL}$ & $\begin{array}{c}50 \mathrm{~mL} / \\
\text { hour }\end{array}$ & $\begin{array}{c}\text { For } 30 \\
\text { minutes } \\
\text { hour for } 30 \\
\text { minutes then } \\
\text { increase by } \\
100 \mathrm{~mL} / \\
\text { hour every } 30 \\
\text { minutes }\end{array}$ & $\begin{array}{c}200 \mathrm{~mL} / \\
\text { hour }\end{array}$ & $\begin{array}{c}200 \mathrm{~mL} / \\
\text { hour }\end{array}$ \\
\hline $\begin{array}{l}\text { Subsequent } \\
\text { infusions }\end{array}$ & $250 \mathrm{~mL}$ & $\begin{array}{c}200 \\
\mathrm{~mL} / \\
\text { hour }\end{array}$ & - & - & \multicolumn{2}{|c|}{} \\
\hline
\end{tabular}

\section{CONTRAINDICATIONS}

SARCLISA is contraindicated in patients with severe hypersensitivity to isatuximab-irfc or to any of its excipients [see Warnings and Precautions (5.1)].

\section{WARNINGS AND PRECAUTIONS}

\section{.1 Infusion-Related Reactions}

Infusion-related reactions have been observed in 39\% of patients treated with SARCLISA [see Adverse Reactions 6.1)]. All infusion-related reactions started during the first SARCLISA infusion and resolved on the same day in $98 \%$ of the cases. The most common symptoms of an infusionrelated reaction included dyspnea, cough, chills, and nausea. The most common severe signs and symptoms included hypertension and dyspnea [see Adverse Reactions (6.1)] To decrease the risk and severity of infusion-related reactions, premedicate patients prior to SARCLISA infusion with acetaminophen, $\mathrm{H} 2$ antagonists, diphenhydramine, or equivalent; dexamethasone [see Dosage and Administration (2.2)]. Monitor vital signs frequently during the entire SARCLISA infusion. For patients with grade 1 or 2 reactions, interrupt SARCLISA infusion and provide appropriate medica support. If symptoms improve, restart SARCLISA infusion at half of the initial infusion rate, with supportive care as needed, and closely monitor patients. If symptoms do not recur after 30 minutes, the infusion rate may be increased to the initial rate, and then increased incrementally, as shown in Table 2 [see Dosage and Administration (2.5)]. In case symptoms do not improve or recur after interruption, permanently discontinue SARCLISA and institute appropriate management. Permanently discontinue SARCLISA therapy if a grade 3 or higher infusion-related reaction occurs and institute appropriate medical management.

\subsection{Neutropenia}

SARCLISA may cause neutropenia. Neutropenia (reported as laboratory abnormality) occurred in $96 \%$ of patients and grade 3-4 neutropenia occurred in $85 \%$ of patients treated with SARCLISA, pomalidomide, and dexamethasone (Isa-Pd). Febrile neutropenia occurred in $12 \%$ of patients and neutropenic infections, defined as infection with concurrent grade $\geq 3$ neutropenia, occurred in $25 \%$ of patients treated with Isa-Pd. The most frequent neutropenic infections included those of upper respiratory tract (10\%), lower respiratory tract (9\%), and urinary tract (3\%) [see Adverse Reactions (6.1)].

Monitor complete blood cell counts periodically during treatment. Consider the use of antibiotics and antiviral prophylaxis during treatment. Monitor patients with neutropenia for signs of infection. In case of grade 4 neutropenia delay SARCLISA dose until neutrophil count recovery to at least $1.0 \times 10^{9} / \mathrm{L}$, and provide supportive care with growth factors, according to institutional guidelines. No .

\subsection{Second Primary Malignancies}

Second primary malignancies were reported in $3.9 \%$ of patients in the SARCLISA, pomalidomide and dexamethasone (Isa-Pd) arm and in $0.7 \%$ of patients in the pomalidomide and dexamethasone $(\mathrm{Pd})$ arm, and consisted of skin squamous cell carcinoma ( $2.6 \%$ of patients in the Isa-Pd arm and in $0.7 \%$ of patients in the Pd arm), breast angiosarcoma $(0.7 \%$ of patients in the Isa-Pd arm) and myelodysplastic syndrome
$(0.7 \%$ of patients in the Isa-Pd arm). With the exception of the patient with myelodysplastic syndrome, patients were able to continue SARCLISA treatment. Monitor patients for the development of second primary malignancies, as per International Myeloma Working Group (IMWG) guidelines. $\mathbf{5 . 4}$ Laboratory Test Interference

Test SARCLISA binds to CD38 on red blood cells (RBCS) and may result in a false positive indirect antiglobulin test (indirect Coombs test). In ICARIA-multiple myeloma (MM) the indirect antiglobulin test was positive during SARCLISA treatment in $67.7 \%$ of the tested patients. In patients with a positive indirect antiglobulin test, blood transfusions were administered without evidence of hemolysis. ABO/RhD typing was not affected by SARCLISA treatment. Before the first SARCLISA infusion, conduct blood type and screen tests on SARCLISA-treated patients. Consider phenotyping prior to starting SARCLISA treatment. If treatment with SARCLISA has already started, inform the blood bank that the patient is receiving SARCLISA and SARCLISA interference with blood compatibility testing can be resolved using dithiothreitoltreated RBCs. If an emergency transfusion is required, noncross-matched ABO/RhD-compatible RBCs can be given as per local blood bank practices [see Drug Interactions (7.1)]. nterference with Serum Protein Electrophoresis and

\section{mmunofixation Tests}

SARCLISA is an IgG kappa monoclonal antibody that can be incidentally detected on both serum protein electrophoresis and immunofixation assays used for the clinical monitoring of endogenous M-protein. This interference can impact the accuracy of the determination of complete response in some patients with IgG kappa myeloma protein [see Drug Interactions (7.1)].

\subsection{Embryo-Fetal Toxicity}

Based on the mechanism of action, SARCLISA can cause feta harm when administered to a pregnant woman. SARCLISA may cause fetal immune cell depletion and decreased bone density. Advise pregnant women of the potential risk to a fetus. Advise females with reproductive potential to use an effective method of contraception during treatment with SARCLISA and for at least 5 months after the last dose [see Use in Specific Populations $(8.1,8.3)]$. The combination of SARCLISA with pomalidomide is contraindicated in pregnant women because pomalidomide may cause birth defects and death of the unborn child. Refer to the pomalidomide

prescribing information on use during pregnancy.

\section{ADVERSE REACTIONS}

The following clinically signiticant adverse reactions from SARCLISA are also described in other sections of the labeling: - Infusion-Related Reactions [see Warnings and Precautions (5.1)]

- Neutropenia [see Warnings and Precautions (5.2)]

- Second Primary Malignancies [see Warnings and

\section{Precautions (5.3)]}

\section{Clinical Trials Experience}

Because clinical trials are conducted under widely varying conditions, adverse reaction rates observed in the clinical trials of a drug cannot be directly compared to rates in the clinical trials of another drug and may not reflect the rates observed in practice.

Multiple Myeloma

The safety of SARCLISA was evaluated in ICARIA-MM a randomized, open-label clinical trial in patients with previously treated multiple myeloma. Patients were eligible for inclusion if they had ECOG status of $0-2$, platelets $\geq 75,000$ cells $/ \mathrm{mm}^{3}$, absolute neutrophil count $\geq 1 \times 10^{9} / \mathrm{L}$, creatinine clearance $\geq 30 \mathrm{~mL} / \mathrm{min}$ (MDRD formula), and AST and/ or ALT $\leq 3 \times$ ULN. Patients received SARCLISA $10 \mathrm{mg} / \mathrm{kg}$ intravenously, weekly in the first cycle and every two weeks thereafter, in combination with pomalidomide and low dose dexamethasone (Isa-Pd) $(n=152)$ or pomalidomide and low dose dexamethasone (Pd) (n=149) [see Clinical Studies (14) in the full prescribing information]. Among patients receiving Isa-Pd, 66\% were exposed to SARCLISA for 6 months or longe and $24 \%$ were exposed for greater than 12 months or longer. The median age of patients who received Isa-Pd was 68 years (range 36-83); 58\% male, 76\% white, and 14\% Asian. Serious adverse reactions occurred in $62 \%$ of patients receiving Isa-Pd. Serious adverse reactions in $>5 \%$ of patients who received lsa-Pd included pneumonia (26\%), upper respiratory tract infections (7\%), and febrile neutropenia (7\%). Fatal adverse reactions occurred in $11 \%$ of patients (those that occurred in more than $1 \%$ of patients were pneumonia and other infections [3\%])

Permanent discontinuation due to an adverse reaction (grades 1-4) occurred in 7\% of patients who received Isa-Pd. The most frequent adverse reactions requiring permanent discontinuation in patients who received Isa-Pd were infections (2.6\%). In addition, SARCLISA alone was discontinued in 3\% of patients due to infusion-related reactions.

Dosage interruptions due to an adverse reaction occurred in $31 \%$ of patients who received SARCLISA. The most frequent adverse reaction requiring dosage interruption was infusionrelated reaction ( $28 \%)$ 
The most common adverse reactions ( $\geq 20 \%)$ were neutropenia, infusion-related reactions, pneumonia, upper respiratory tract infection, and diarrhea.

Table 3 summarizes the adverse reactions in ICARIA-MM

Table 3: Adverse Reactions ( $\mathbf{1 0} \%$ ) in Patients

Receiving SARCLISA, Pomalidomide, and Dexamethasone with a Difference Between Arms of $\geq 5 \%$ Compared to Control Arm in ICARIA-MM Trial

\begin{tabular}{|c|c|c|c|c|c|c|}
\hline \multirow[t]{2}{*}{$\begin{array}{l}\text { Adverse } \\
\text { Reactions }\end{array}$} & \multicolumn{3}{|c|}{$\begin{array}{c}\text { SARCLISA+ } \\
\text { Pomalidomide + } \\
\text { Dexamethasone (Isa-Pd) } \\
(\mathrm{N}=152)\end{array}$} & \multicolumn{3}{|c|}{$\begin{array}{c}\text { Pomalidomide + } \\
\text { Dexamethasone (Pd) } \\
(\mathrm{N}=149)\end{array}$} \\
\hline & $\begin{array}{c}\text { All } \\
\text { grades } \\
(\%)\end{array}$ & $\begin{array}{c}\text { Grade } \\
3 \\
(\%)\end{array}$ & $\begin{array}{c}\text { Grade } \\
4 \\
(\%)\end{array}$ & $\begin{array}{c}\text { All } \\
\text { grades } \\
(\%)\end{array}$ & $\begin{array}{c}\text { Grade } \\
3 \\
(\%)\end{array}$ & $\begin{array}{c}\text { Grade } \\
4 \\
(\%)\end{array}$ \\
\hline $\begin{array}{l}\text { Infusion-related } \\
\text { reaction }\end{array}$ & 38 & 1.3 & 1.3 & 0 & 0 & 0 \\
\hline \multicolumn{7}{|l|}{ Infections } \\
\hline Pneumonia* & 31 & 22 & 3.3 & 23 & 16 & 2.7 \\
\hline $\begin{array}{l}\text { Upper } \\
\text { respiratory tract } \\
\text { infection }\end{array}$ & 57 & 9 & 0 & 42 & 3.4 & 0 \\
\hline \multicolumn{7}{|c|}{ Blood and lymphatic system disorders } \\
\hline $\begin{array}{l}\text { Febrile } \\
\text { neutropenia }\end{array}$ & 12 & 11 & 1.3 & 2 & 1.3 & 0.7 \\
\hline \multicolumn{7}{|c|}{ Respiratory, thoracic and mediastinal disorders } \\
\hline Dyspnea $^{\ddagger}$ & 17 & 5.0 & 0 & 12 & 1.3 & 0 \\
\hline \multicolumn{7}{|c|}{ Gastrointestinal disorders } \\
\hline Diarnhea & 26 & 2 & - & 19 & 0.7 & - \\
\hline Nausea & 15 & 0 & - & 9 & 0 & - \\
\hline Vomiting & 12 & 1.3 & - & 3.4 & 0 & - \\
\hline
\end{tabular}

CTCAE version 4.03

*Pneumonia includes atypical pneumonia, bronchopulmonary aspergillosis, pneumonia, pneumonia haemophilus, pneumonia influenzal, pneumonia pneumococcal, pneumonia streptococcal, pneumonia viral, candida pneumonia, pneumonia bacterial, haemophilus infection, lung infection, pneumonia fungal, and pneumocystis jirovecii pneumonia.

+Upper respiratory tract infection includes bronchiolitis, bronchitis, bronchitis viral, chronic sinusitis,

fungal pharyngitis, influenza-like illness, laryngitis,

nasopharyngitis, parainfluenzae virus infection, pharyngitis, respiratory tract infection, respiratory tract infection viral

rhinitis, sinusitis, tracheitis, upper respiratory tract infection, and upper respiratory tract infection bacterial.

†Dyspnea includes dyspnea, dyspnea exertional, and dyspnea at rest.

Table 4 summarizes the hematology laboratory abnormalities in ICARIA-MM.

Table 4: Treatment Emergent Hematology Laboratory Abnormalities in Patients Receiving Isa-Pd Treatment versus Pd Treatment - ICARIA-MM

\begin{tabular}{|l|c|c|c|c|c|c|}
\hline $\begin{array}{l}\text { Laboratory } \\
\text { Parameter } \\
\text { n (\%) }\end{array}$ & \multicolumn{3}{|c|}{$\begin{array}{c}\text { SARCLSA+ } \\
\text { Pomalidomide+ } \\
\text { Dexamethasone (Isa-Pd) } \\
\text { (N=152) }\end{array}$} & \multicolumn{3}{|c|}{$\begin{array}{c}\text { Pomalidomide + } \\
\text { Dexamethasone (Pd) } \\
\text { (N=149) }\end{array}$} \\
\hline & $\begin{array}{c}\text { All } \\
\text { Grades }\end{array}$ & $\begin{array}{c}\text { Grade } \\
\mathbf{3}\end{array}$ & $\begin{array}{c}\text { Grade } \\
\mathbf{4}\end{array}$ & $\begin{array}{c}\text { All } \\
\text { Grades }\end{array}$ & $\begin{array}{c}\text { Grade } \\
\mathbf{3}\end{array}$ & $\begin{array}{c}\text { Grade } \\
\mathbf{4}\end{array}$ \\
\hline Anemia & 151 & 48 & 0 & $\begin{array}{c}145 \\
(97)\end{array}$ & $\begin{array}{c}41 \\
(28)\end{array}$ & 0 \\
\hline Neutropenia & 146 & 37 & 92 & 137 & 57 & 46 \\
$(32)$ & $(96)$ & $(24)$ & $(61)$ & $(92)$ & $(38)$ & $(31)$ \\
\hline Lymphopenia & 140 & $\begin{array}{c}64 \\
(42)\end{array}$ & $\begin{array}{c}19 \\
(13)\end{array}$ & $\begin{array}{c}137 \\
(92)\end{array}$ & $\begin{array}{c}52 \\
(35)\end{array}$ & $\begin{array}{c}12 \\
(8)\end{array}$ \\
\hline Thrombocytopenia & $\begin{array}{c}127 \\
(84)\end{array}$ & $\begin{array}{c}22 \\
(14)\end{array}$ & $\begin{array}{c}25 \\
(16)\end{array}$ & $\begin{array}{c}118 \\
(79)\end{array}$ & $\begin{array}{c}14 \\
(9)\end{array}$ & $\begin{array}{c}22 \\
(15)\end{array}$ \\
\hline
\end{tabular}

Description of Selected Adverse Reactions

\section{infusion-related reactions}

In ICARIA-MM, infusion-related reactions (defined as adverse reactions associated with the SARCLISA infusions, with an onset typically within 24 hours from the start of the infusion) were reported in 58 patients (38\%) treated with SARCLISA. All patients who experienced infusion-related reactions, experienced them during the 1st infusion of SARCLISA, with 3 patients (2\%) also having infusion-related reactions at thei 2nd infusion, and 2 patients (1.3\%) at their 4th infusion. Grade 1 infusion-related reactions were reported in 3.9\%, Grade 2 in 32\%, Grade 3 in 1.3\%, and Grade 4 in 1.3\% of the patients. Signs and symptoms of Grade 3 or higher infusion-related reactions included dyspnea, hypertension, and bronchospasm. The incidence of infusion interruptions because of infusion-related reactions was $29.6 \%$. The median time to infusion interruption was 55 minutes.

In a separate study (TCD 14079 Part B) with SARCLISA 10 $\mathrm{mg} / \mathrm{kg}$ administered from a $250 \mathrm{~mL}$ fixed-volume infusion in combination with $\mathrm{Pd}$, infusion-related reactions (all Grade 2) were reported in $40 \%$ of patients, at the first administration, the day of the infusion. Overall, the infusion-related reactions of SARCLISA $10 \mathrm{mg} / \mathrm{kg}$ administered as a $250 \mathrm{~mL}$ fixed-volume infusion were similar to that of SARCLISA as administered in ICARIA-MM.

intections

In ICARIA-MM, the incidence of Grade 3 or higher infections was $43 \%$ in Isa-Pd group. Pneumonia was the most commonly reported severe infection with Grade 3 reported in $22 \%$ of patients in Isa-Pd group compared to $16 \%$ in $\mathrm{Pd}$ group, and Grade 4 in 3.3\% of patients in Isa-Pd group compared to $2.7 \%$ in Pd group. Discontinuations from treatment due to infection were reported in $2.6 \%$ of patients in Isa-Pd group compared to $5.4 \%$ in Pd group. Fatal infections were reported in $3.3 \%$ of patients in Isa-Pd group and in $4 \%$ in $\mathrm{Pd}$ group

\subsection{Immunogenicity}

As with all therapeutic proteins, there is a potential for immunogenicity. The detection of antibody formation is highly dependent on the sensitivity and specificity of the assay. Additionally, the observed incidence of antibody (including neutralizing antibody) positivity in an assay may be influenced by several factors, including assay methodology, sample handling, timing of sample collection, concomitant medications, and underlying disease. For these reasons, comparison of the incidence of antibodies in the studies described below with the incidence of antibodies in other studies or to other isatuximab-irfc products may be misleading

In ICARIA-MM, no patients tested positive for antidrug antibodies (ADA). Therefore, the neutralizing ADA status was not determined. Overall, across 6 clinical studies in multiple myeloma (MM) with SARCLISA single agent and combination therapies including ICARIA-MM ( $\mathrm{N}=564)$, the incidence of treatment emergent ADAs was $2.3 \%$. No clinically significant differences in the pharmacokinetics, safety, or efficacy of isatuximab-irfc were observed in patients with ADAs.

\section{DRUG INTERACTIONS}

\subsection{Daboratory Test interference}

Interference with Serological Testing

SARCLISA, an anti-CD38 antibody, may interfere with blood bank serologic tests with false positive reactions in indirect antiglobulin tests (indirect Coombs tests), antibody detection (screening) tests, antibody identification panels, and antihuman globulin crossmatches in patients treated with SARCLISA [see Warnings and Precautions (5.4)]. nterference with Serum Protein Electrophoresis and Immunofixation Tests

SARCLISA may be incidentally detected by serum protein electrophoresis and immunofixation assays used for the monitoring of M-protein and may interfere with accurate response classification based on International Myeloma Working Group (IMWG) criteria [see Warnings and Precautions (5.4)].

\section{USE IN SPECIFIC POPULATIONS}

\subsection{Pregnancy}

\section{Risk Summary}

SARCLISA can cause fetal harm when administered to a pregnant woman. The assessment of isatuximab-irfoassociated risks is based on the mechanism of action and data from target antigen CD38 knockout animal models (see Data). There are no available data on SARCLISA use in pregnant women to evaluate for a drug-associated risk of major birth defects, miscarriage or adverse maternal or fetal outcomes. Animal reproduction toxicity studies have not been conducted with isatuximab-irfc. The estimated background risk of major birth defects and miscarriage for the indicated population is unknown. All pregnancies have a background risk of birth defect, miscarriage, or other adverse outcomes. In the U.S. general population, the estimated background risk of major birth defects and miscarriage in clinically recognized pregnancies is $2 \%$ to $4 \%$ and $15 \%$ to $20 \%$, respectively. The combination of SARCLISA and pomalidomide is contraindicated in pregnant women because pomalidomide may cause birth defects and death of the unborn child. Refer to the pomalidomide prescribing information on use during pregnancy. Pomalidomide is only available through a REMS program.

Clinical Considerations

Fetal/neonatal reactions

Immunoglobulin G1 monoclonal antibodies are known to cross the placenta. Based on its mechanism of action, SARCLISA may cause depletion of fetal CD38-positive immune cells and decreased bone density. Defer administration of live vaccines to neonates and infants exposed to SARCLISA in utero until a hematology evaluation is completed. (isatuximab-irfc) injection, for intravenous use

Data

Mice that were genetically modified to eliminate all CD38 expression (CD38 knockout mice) had reduced bone density which recovered 5 months after birth. Data from studies using CD38 knockout animal models also suggest the involvement of CD38 in regulating humoral immune responses (mice), feto-maternal immune tolerance (mice), and early embryonic development (frogs).

\subsection{Lactation}

\section{Risk Summary}

There are no available data on the presence of isatuximab-iffc in human milk, milk production, or the effects on the breastfed child. Maternal immunoglobulin $\mathrm{G}$ is known to be present in human milk. The effects of local gastrointestinal exposure and limited systemic exposure in the breastfed infant to SARCLISA are unknown. Because of the potential for serious adverse reactions in the breastfed child from isatuximab-iffc administered in combination with pomalidomide and dexamethasone, advise lactating women not to breastfeed during treatment with SARCLISA. Refer to pomalidomide prescribing information for additional information.

\subsection{Females and Males of Reproductive Potential}

Pregnancy Testing

With the combination of SARCLISA with pomalidomide, refer to the pomalidomide labeling for pregnancy testing requirements prior to initiating treatment in females of reproductive potential.

Contraception

Females

SARCLISA can cause fetal harm when administered to a pregnant woman [see Use in Specific Populations (8.1)]. Advise female patients of reproductive potential to use effective contraception during treatment and for at least 5 months after the last dose of SARCLISA. Additionally, refer to the pomalidomide labeling for contraception requirements prior to initiating treatment in females of reproductive potential

Males

Refer to the pomalidomide prescribing information.

\subsection{Pediatric Use}

Safety and effectiveness in pediatric patients have not been established.

\subsection{Geriatric Use}

Of the total number of subjects in clinical studies of

SARCLISA, 53\% (306 patients) were 65 and over, while 14\% (82 patients) were 75 and over. No overall differences in safety or effectiveness were observed between subjects 65 and ove and younger subjects, and other reported clinical experience has not identified differences in responses between the adults 65 years and over and younger patients, but greater sensitivity of some older individuals cannot be ruled out.

\section{OVERDOSAGE}

There is no known specific antidote for SARCLISA overdose. In the event of overdose of SARCLISA, monitor the patients for signs or symptoms of adverse effects and take all appropriate measures immediately.

Manufactured by:

sanofi-aventis U.S. LLC

Bridgewater, NJ 08807

U.S. License No. 1752

SARCLISA is a registered trademark of Sanofi

(C)2020 sanofi-aventis U.S. LLC

ISA-BPLR-SA-MAR20

Revised: March 2020 


\section{FDA Approves Pembrolizumab + Chemotherapy Combination for Locally Recurrent Unresectable or Metastatic TNBC}

Hannah Slater

$\mathbf{T}$ he FDA has granted accelerated approval to pembrolizumab (Keytruda) in combination with chemotherapy for the treatment of patients with locally recurrent unresectable or metastatic triple-negative breast cancer (TNBC) whose tumors express PDL1 (combined positive score [CPS] $\geq 10$ ) as determined by an FDA approved test. ${ }^{1}$

The FDA approval was based on data from the multicenter, double-blind, randomized, placebo-controlled, phase 3 KEYNOTE-355 trial (NCT02819518), which evaluated patients with locally recurrent unresectable or metastatic TNBC who had not been previously treated with chemotherapy in the metastatic setting.

Patients were randomized 2:1 to receive $200 \mathrm{mg}$ of pembrolizumab or placebo on day 1 every 3 weeks in combination with different chemotherapy treatments (paclitaxel protein-bound, or paclitaxel, or gemcitabine plus carboplatin) via intravenous infusion. The primary end point for the study was progression-free survival (PFS) as assessed by blinded independent review according to RECIST 1.1, tested in the subgroup of patients with CPS of 10 or higher.

The median PFS was 9.7 months $(95 \%$ CI, 7.6-11.3) in the pembrolizumab plus chemotherapy arm and 5.6 months
(95\% CI, 5.3-7.5) in the placebo plus chemotherapy arm (HR, 0.65; 95\% CI, 0.49-0.86; 1 -sided $P=.0012$ ).

Regarding safety, the most common adverse events observed in patients receiving pembrolizumab plus chemotherapy in KEYNOTE-355 ( $220 \%$ of patients) were fatigue, nausea, diarrhea, constipation, vomiting, alopecia, rash, cough, decreased appetite, and headache. Further, the most common laboratory abnormalities in patients receiving pembrolizumab plus chemotherapy (incidence $\geq 20 \%$ ) were anemia, leukopenia, neutropenia, lymphopenia, thrombocytopenia, elevated liver enzymes, hyperglycemia, hypoalbuminemia, increased alkaline phosphatase, hypocalcemia, hyponatremia, hypophosphatemia, and hypokalemia.

The FDA recommended dose of pembrolizumab for adult patients with locally recurrent unresectable or metastatic TNBC is $200 \mathrm{mg}$ every 3 weeks or 400 mg every 6 weeks administered prior to chemotherapy until disease progression, unacceptable toxicity, or up to 24 months. When given with pembrolizumab, either paclitaxel protein-bound $100 \mathrm{mg} / \mathrm{m}^{2}$ on days 1,8 , and 15 every 28 days; or paclitaxel $90 \mathrm{mg} / \mathrm{m}^{2}$ on days 1,8 , and 15 every 28 days; or gemcitabine $1000 \mathrm{mg} / \mathrm{m}^{2}$ plus carboplatin area under the curve $2 \mathrm{mg} / \mathrm{mL} / \mathrm{min}$ on days 1 and 8 every 21 days should be administered via intravenous infusion.

Of note, this application was granted accelerated approval based on PFS. However, continued approval for this indication may be dependent upon verification and description of clinical benefit in the confirmatory trials.

The FDA also approved the PD-L1 IHC 22C3 pharmDx as a companion diagnostic for selecting patients with TNBC whose tumors express PD-L1 with a CPS of 10 or higher. ${ }^{2}$

Currently, PD-L1 IHC 22C3 pharm$\mathrm{Dx}$ is the only companion diagnostic that is FDA approved to assist in the identification of patients with TNBC who would benefit from treatment with pembrolizumab in combination with chemotherapy. This marks the seventh cancer type for which PD-L1 IHC 22C3 pharmDx has been granted FDA approval; the diagnostic also helps physicians identify patients with non-small cell lung cancer, gastric or gastroesophageal junction adenocarcinoma, esophageal squamous cell carcinoma, cervical cancer, urothelial carcinoma, and head and neck squamous cell carcinoma who would benefit.

For full reference list, visit cancernetwork.com/Pembro_TNBC 


\section{Pancreatic Neuroendocrine Tumor With Humoral Hypercalcemia and High Tumor PD-L1 Score}

Mehmet Sitki Copur MD, FACP'; Luciano Vargas MD²; Whitney Wedel MD³; Shaheed Merani MD²; Allison Cushman-Vokoun $\mathrm{MD}^{4}, \mathrm{PhD}$; Angela Drincic MD ${ }^{5}$

'Mary Lanning Healthcare Morrison Cancer Center Hastings Nebraska, University of Nebraska Medical Center, Omaha, Nebraska; ${ }^{2}$ Transplant and General Surgery, University of Nebraska Medical Center, Omaha, Nebraska; ${ }^{3}$ Mary Lanning Healthcare Anatomic Pathology. Hastings, Nebraska; ${ }^{2}$ Pathology Microbiology Molecular Diagnostics, University of Nebraska Medical Center, Omaha, Nebraska; ${ }^{5}$ Diabetes and Endocrinology Center, University of Nebraska Medical Center Omaha, Nebraska

\begin{abstract}
Pancreatic neuroendocrine tumors (NETs) are a heterogeneous group of neoplasms. They can be functioning tumors with secretion of a variety of peptide hormones, or nonfunctioning tumors with metastases to the liver at the time of diagnosis. Well-differentiated tumors tend to be slow-growing and characterized by low tumor mutational burden (TMB) and lower propensity to express PD-L1. Hypercalcemia due to malignancy can occur in about $20 \%$ to $30 \%$ of patients with cancer. The secretion of parathyroid hormone-related protein (PTH-rP) is among the causes of malignant hypercalcemia and has seldom been associated with hypercalcemia of NETs. Although the therapeutic landscape for neuroendocrine neoplasms has evolved substantially over the past decade, the role of immunotherapy has not yet been completely explored in this group of patients. We present a rare case of a metastatic pancreatic NET with high TMB, high PD-L1 tumor proportion score, and high PTH-rPrelated hypercalcemia.
\end{abstract}

\section{Introduction}

Pancreatic neuroendocrine tumors (NETs) are rare. They have an incidence of less than 1 case per 100,000 individuals per year. Pancreatic NETs are generally indolent and carry a much better prognosis than ductal adenocarcinoma of the pancreas. ${ }^{1-3}$ Although pancreatic NETs account for approximately $1 \%$ to $3 \%$ of all pancreatic cancers by prevalence, they constitute $10 \%$ of all pancreatic cancers due to their better survival. ${ }^{4}$ The majority (40\%-91\%) of pancreatic NETs are nonfunctional, but the remainder can present with clinically evident hormonal symptoms depending on the hormones they secrete. Of the functioning tumors, $70 \%$ are insulinomas, $15 \%$ are glucagonomas, and $10 \%$ are gastrinomas and somatostatinomas. The remaining rare functional pancreatic NETs include vipomas and cholecystokinin-secreting tumors. ${ }^{5-7}$

Hypercalcemia is a well-known com- plication of cancer and can be caused by local osteolytic effects of cancer, excess $1,25(\mathrm{OH})_{2} \mathrm{D}$ secretion, or ectopic parathyroid hormone secretion. Humoral hypercalcemia of malignancy, which is caused by the oversecretion of parathyroid hormone-related peptide (PTH-rP), is responsible for $80 \%$ of malignancy associated hypercalcemias..$^{8-11}$ Although humoral hypercalcemia can be caused by any tumor, its presence in patients with pancreatic NETs is extremely rare. ${ }^{12-14}$

Immunotherapy in oncology has shown promising results in many cancer types. The immune landscape of NETs has yet to be fully defined. Most NETs appear to be mismatch repair proficient and have a low tumor mutational burden (TMB). Only $3 \%$ of patients with NETs have been reported to harbor more than 17 mutations per megabase. ${ }^{15}$ Immune checkpoint molecules are heterogeneously expressed in the surrounding cells of NETs, and it is unclear whether or not tumor-infiltrating lymphocytes may have antitumor effects within the tumor microenvironment. Recently, an immune checkpoint inhibitor combination therapy showed efficacy in patients with high-grade neuroendocrine carcinoma. ${ }^{16}$ 

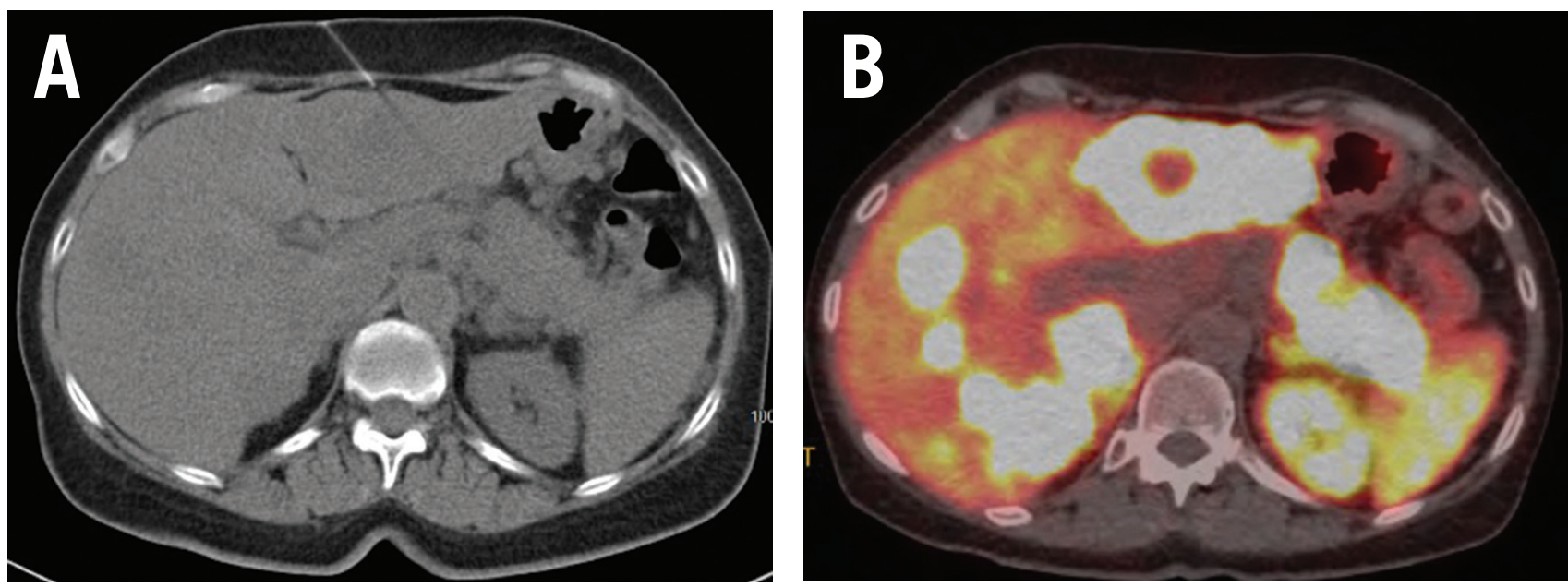

Figure 1 Large necrotic mass in the pancreatic body/tail extending to the splenic hilum with the invasion into spleen. Multiple enhancing lesions throughout the liver (A). Large pancreatic body/tail PET avid mass with direct extension into splenic hilum. Multiple PET avid hepatic metastases (B)

\section{Case}

A White woman aged 62 years noted poor appetite, occasional nausea, vomiting, indigestion, and a 30-pound weight loss starting 6 months prior to her diagnosis. Initial evaluation, including upper and lower endoscopies, revealed Barretts esophagus with no dysplasia, a cecal polyp, and negative celiac disease markers. Treatment with proton pump inhibitors did not help her symptoms. As time passed, she noted occasional epigastric and right upper quadrant abdominal pain. Further evaluation with ultrasound and a CT scan of the abdomen revealed a large necrotic mass in the pancreatic body/ tail extending to the splenic hilum with invasion into the spleen, as well as multiple enhancing lesions throughout the liver (Figure 1A). A CT-guided biopsy of a liver lesion revealed a metastatic, well-differentiated, high-grade (grade 3) NET with rosette formation, high mitotic rate (more than 20 per high power field), and a Ki-67 index of $30 \%$ (Figure 2 A, B). Further work-up with ${ }^{68}$ gallium 68-dotatate PET-CT confirmed a large pancreatic body/tail PET avid mass with direct extension involving the splenic hilum and multiple hepatic metastases.

(Figure 1B). Ultrasound of the thyroid and parathyroid glands and MRI of the brain was negative. Serum gastrin, glucagon, and vasoactive intestinal peptide levels were normal. She had no fasting or nocturnal hypoglycemia. Serum chromogranin A level was elevated at $754 \mathrm{ng} /$ $\mathrm{mL}$ (normal 0-103). A genetic consultation and work-up by our certified genetic counselor did not reveal any familial neuroendocrine syndrome.

Shortly after her diagnosis, the patient was noted to have an elevated calcium level of $12.6 \mathrm{mg} / \mathrm{dL}$. Further work-up revealed normal vitamin D 25(OH) (30.69 ng/dL; normal, 30-60); normal vitamin D 1, 25 $(\mathrm{OH})_{2}(78 \mathrm{pg} / \mathrm{mL}$; normal, 19 $79)$; suppressed intact PTH $(4.6 \mathrm{pg} / \mathrm{mL}$; (normal, 12-78); and elevated PTH-rP (32 pmol/L; normal, 0-4.2). Because of the extent of her disease, she was initially treated with systemic fluoropyrimidine-oxaliplatin-based systemic chemotherapy along with monthly long-acting octreotide acetate (Sandostatin LAR Depot) injections. Calcium level returned to normal with intravenous bisphosphonate and isotonic saline. After the completion of 4 cycles of systemic treatment, she underwent an exploratory laparotomy with the intent of tumor debulking, distal pancreatectomy, and splenectomy. However, the extent of tumor invasion of the celiac plexus, left gastric artery, and splenic artery, which were not predictable by the imaging studies, limited the surgery to a wedge resection of the liver.

PD-L1 immunohistochemistry analysis of the tumor revealed a tumor proportion score (TPS) of $80 \%$. Next-generation sequencing analysis showed a TMB of 15 per megabase, stable microsatellite status, and genomic findings of RNF43 p.G659fs*41,ATRX p.S750del, BCOR p.L855fs*1,HNF1Ap.P379fs*5, MLH1E172,MSH3 p.K383fs*32,QKI p.K134fs*14. She was started on immune checkpoint inhibitor therapy with pembrolizumab and continued on long-acting octreotide acetate.

\section{Discussion}

Our increased understanding of the biology, pathophysiology, and genetics of pancreatic NETs has led to improved diagnostic and treatment options. However, the optimal treatment and sequencing of these treatments remain largely undefined. The relatively uncommon occurrence and indolent nature of some NETs make designing and completing adequately powered randomized trials very difficult. Most patients receive multiple modalities of 


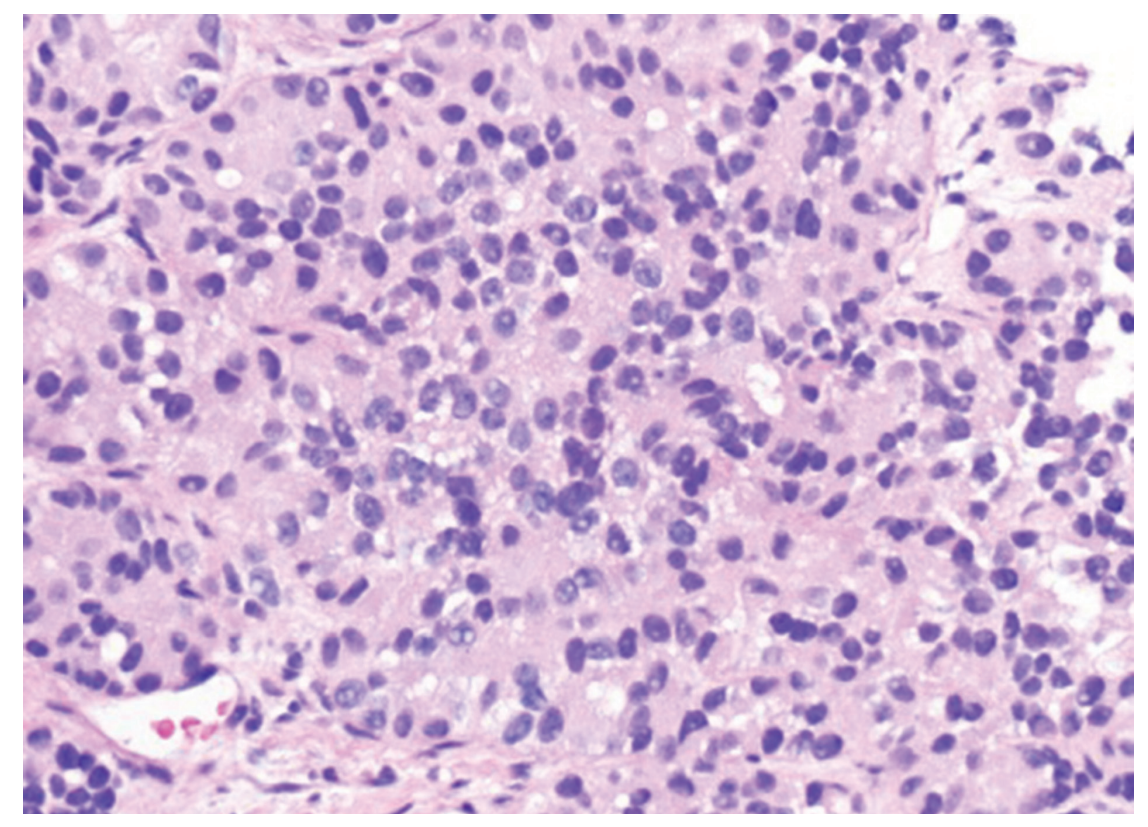

- Figure 2A Pancreatic well-differentiated neuroendocrine tumor. Well-differentiated neoplasm with rosette formation; the Ki67 index was approximately 30\% (H\&E, 400X magnification).

treatments during the course of their disease. Assessment of the effectiveness of a particular treatment relative to the other treatments becomes very challenging due to the use of a plethora of various treatments with widely varied sequencing. Thus, a lack of established data on optimal treatment sequencing exists. Available practice guidelines offer a wide variety of management options but remain inadequate for the care of individual patients. Involvement of an experienced multidisciplinary team is crucial to provide the optimum coordinated care. For patients with metastatic disease, a multitude of treatment options has ranged from surgical debulking, to systemic chemotherapy, targeted therapy, liver-directed therapy, peptide receptor radionuclide therapy, and more.

\section{Surgery}

Surgery is the initial treatment approach for most early-stage tumors. However, patients with incidentally discovered nonfunctioning tumors, especially those less than $1 \mathrm{~cm}$ in size, may be followed depending on the site of the tumor. ${ }^{17,18}$ Resection of the primary tumor and its metastases may be of benefit in selected patients with advanced disease. Metastases in patients with NETs of the pancreas often occur first in the liver. $\mathrm{Cu}-$ rative-intent surgical excision of both the primary tumor and the metastases should be considered in patients with limited hepatic disease. This can be performed in a staged or synchronous fashion. ${ }^{19}$ Noncurative debulking surgery can also be considered in selected cases. When performing staged pancreatoduodenectomy and liver resection, hepatectomy should be considered before pancreatic resection to minimize the risk of perihepatic sepsis from the contaminated biliary tree..$^{20}$ Additional resection and/or ablation may be possible, providing long-term survival. ${ }^{21} \mathrm{~A}$ prophylactic cholecystectomy may be considered, to avoid biliary symptoms and gallstones that could occur due to the continued future use of long-term somatostatin analogues. ${ }^{22}$

\section{Systemic Therapy}

Symptomatic patients with clinically significant unresectable tumor burden may benefit from systemic therapy. Several different cytotoxic chemotherapy regimens have been shown to have antitumor activity but no consensus exists on which regimen is the best. Streptozosin is approved for use in patients with advanced pancreatic NETs. The combination of doxorubicin and streptozosin showed an overall response rate (ORR) of $69 \%$ and a survival benefit in a small randomized trial. ${ }^{23}$ An ORR of $39 \%$ was reported in a retrospective study using the combination of streptozosin, 5 -fluorouracil, and doxorubicin. ${ }^{24}$ In another study with 88 patients, FOLFOX demonstrated an ORR of $31 \%$, median progression-free survival (PFS) of 9 months, and median overall survival (OS) of 30 months,${ }^{25}$ while in a smaller study with FOLFOX, a disease control rate of $78 \%$ was observed. ${ }^{26}$

Octreotide or lanreotide should be considered in certain patients with symptomatic or unresectable disease, high tumor burden, or significant disease progression. Patients with symptoms of hormone secretion or patients who have uptake with somatostatin scintigraphy benefit from somatostatin analogues. Octreotide and lanreotide share the same mechanism of action. In a randomized study of 204 patients with gastroenteropancreatic NETs, treatment with lanreaotide vs placebo resulted in improved PFS (not reached vs 18 months; $P<.001) .{ }^{27}$ Octreotide showed improvement in time to tumor progression (14.3 vs 6 months; $P=.000072$ ) in carcinoid tumors of the midgut. ${ }^{28}$ Radiolabeled somatostatin analogues have shown tumor responses and encouraging survival rates in nonrandomized studies. ${ }^{29,30} \mathrm{In}$ a study involving 610 patients with metastatic gastroenteropancreatic and bronchial NETs, treatment with ${ }^{177} \mathrm{Lu}$-dotatate resulted in complete responses in 6 patients and a 71-month median OS in patients with pancreatic NETs. ${ }^{31}$ 
Oral temozolomide, either alone or in combination, has shown activity in advanced pancreatic NETs. In results of a retrospective series, temozolomide combined with capecitabine showed an objective response rate of $70 \%$ and a median PFS of 18 months. ${ }^{32}$ Kunz et al presented prospective randomized phase 2 trial data comparing single-agent temozolomide with the combination of temozolomide and capecitabine in advanced pancreatic NETs; the combination arm showed improved PFS and OS. ${ }^{33}$ In a prospective study combination of temozolomide with bevacizum$a b$, radiographic response was seen in $33 \%$ of patients, ${ }^{34}$ and in another study the combination of temozolomide with everolimus showed partial response in $40 \%$ of patients with pancreatic neuroendocrine tumors. ${ }^{35}$ Everolimus was evaluated in a randomized multicenter study involving 410 patients with advanced progressive pancreatic NETs, and the agent showed PFS benefit compared with placebo (11.0 vs 4.6 months; $P<.001) .{ }^{36}$ Sunitinib was compared with placebo in a randomized study of 171 patients and also showed a PFS advantage over placebo (11.4 vs 5.5 months; $P<.001) .{ }^{37}$

\section{Liver-Directed Therapies}

Various liver-directed therapies have been available for hepatic-predominant metastatic disease, mostly to reduce the tumor bulk and relieve symptoms of hormone hypersecretion. Among these are transarterial embolization, chemoembolization, radioembolization, radiofrequency ablation, cryotherapy, and microwave ablation. Embolization in general is considered an effective approach in patients with liver-predominant disease ${ }^{38-40}$; however, there are limited data comparing various embolization techniques. A systematic review of radiofrequency ablation of $301 \mathrm{pa}-$ tients reported symptom improvement of $92 \%$, with a median duration of 14 to 27 months. ${ }^{41}$

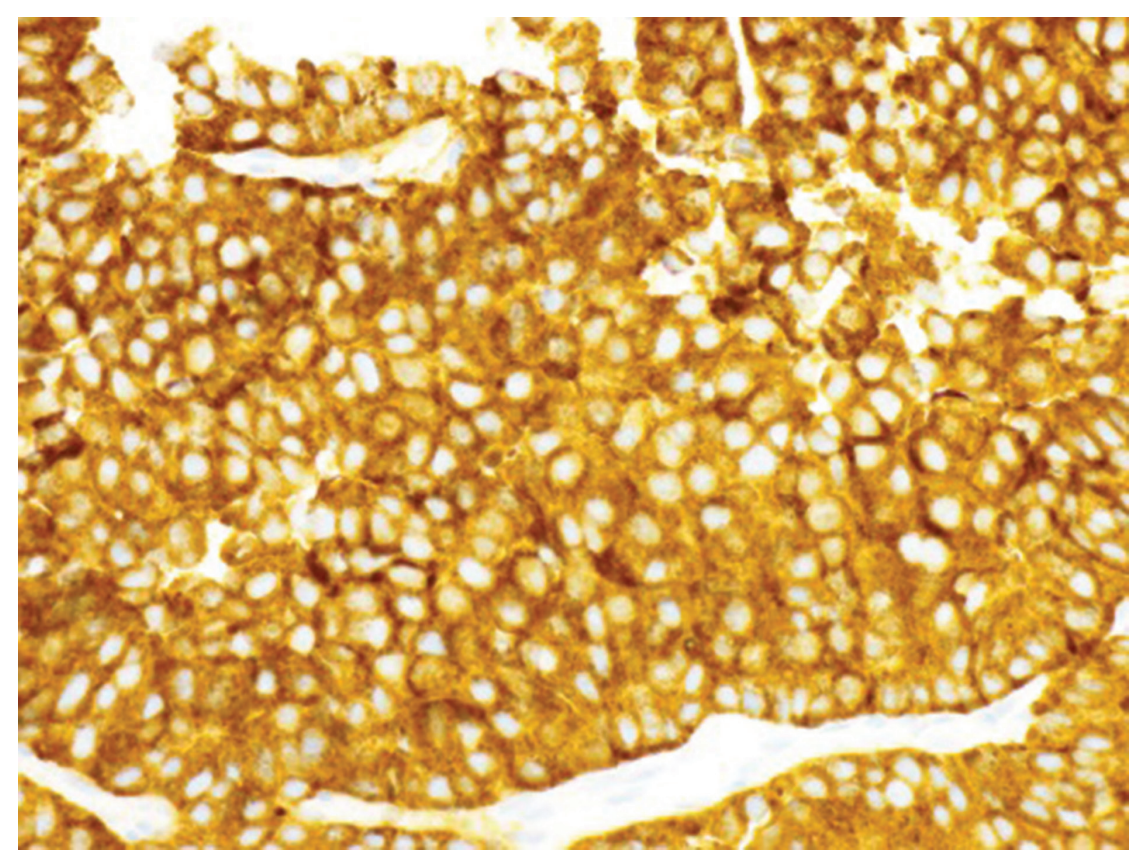

Figure 2B Positivity for synaptophysin (immunoperoxidase stain, 400X magnification).

Microwaves are a nonionizing form of radiation that cause extremely rapid oscillation of the water within tissues; friction from the fluctuation of intracellular water molecules generates heat, which in turn leads to coagulative necrosis. The intratumoral temperatures of microwave ablation are consistently higher than those that can be achieved with radiofrequency ablation. In both radiofrequency ablation and microwave ablation, a probe is placed into the target lesion under radiographic guidance or during open surgery. Multiple lesions can be ablated during the same procedure. However, here is a paucity of data comparing microwave ablation with radiofrequency ablation for management of NETs in the liver. ${ }^{42,43}$

Cryoablation, another thermoablation technique, works by decreasing cell viability through low temperatures. ${ }^{44}$ Seifert et al described a series of 13 patients with NETs who underwent hepatic cryotherapy; cryoprobes were inserted under ultrasound guidance. Twelve of 13 patients had complete ablation of all visible tumors, with 2 recurrences at the ablation sites and 12 survivors at 1 year of post-ablation. All 7 patients who had hormonally related symptoms prior to cryotherapy experienced palliative benefit. Two patients developed a postprocedural coagulopathy requiring intraabdominal packing and the transfusion of clotting factors. ${ }^{45}$

\section{Unusual Features of the Case High TMB With a High PD-L1 TPS}

PD-L1 immunohistochemistry analysis of the tumor in our patient revealed a TPS of $80 \%$, and next-generation sequencing analysis indicated a TMB of 15 per megabase. The role of immunotherapy in NET patients has not been well defined. Predictive biomarkers such as high TMB and high PD-L1 TPS are being evaluated in identifying patients who might benefit from this treatment modality. Our current knowledge about immunotherapy in gastroenteropancreatic NETs mostly comes from early-phase clinical trials and case reports. In the phase $1 \mathrm{~b}$ KEYNOTE-028 study, 16 patients with pancreatic NETs who 
were treated with pembrolizumab monotherapy had an ORR of $6 \%$, PFS of $27 \%$ at 12 months, and a median OS of $87 \% .{ }^{46}$ The analysis of data from a phase 2 basket trial of atezolizumab and bevacizumab in solid tumors, which included pancreatic and extrapancreatic NET cohorts and involved 40 patients with previously treated grade $1 / 2$ NETs, revealed ORRs of $20 \%$ and $15 \%$ and median PFS of 19.6 months and 14.9 months in the 2 cohorts, respectively. ${ }^{47}$ In another phase 2 basket trial, a dual anti-CTLA-4 and anti-PD-1 blockade was tested by utilizing nivolumab plus ipilimumab in 15 patients with nonpancreatic gastrointestinal NETs. ${ }^{16}$ Objective responses were observed only in high-grade NETs with an ORR of $44 \%$, but the blockade had meager efficacy in the well-differentiated tumors. Several case reports of the use of single-agent or combination immunotherapy in NETs have also been published. ${ }^{48-51}$ Our patient's high TMB, high PD-L1 score, and grade 3 histology led us to utilize immunotherapy.

\section{Elevated PTH-rP and Hypercalcemia}

An elevated calcium level with a suppressed intact PTH level and an elevated PTH-rP level was another rather unusual feature of our case. Malignancy-relat- ed hypercalcemia is rather uncommon in pancreatic NETs. ${ }^{52-54}$ PTH-rP is an autocrine, paracrine, and endocrine peptide involving calcium transportation and/or signaling expressed in response to a variety of physiological stimuli. ${ }^{55}$ It is structurally similar to PTH and, like PTH, it enhances renal tubular reabsorption of calcium while simultaneously increasing urinary phosphorus excretion. However, unlike PTH, it does not increase 1 , $25(\mathrm{OH})_{2} \mathrm{D}$, thus it does not increase intestinal absorption of calcium and phosphorus. PTH-rP acts on osteoblasts, leading to enhanced synthesis of the receptor activator nuclear kappa B (RANK) ligand. ${ }^{56,57}$ Bisphosphonates can normalize serum calcium level in more than $70 \%$ of patients; however, in approximately $25 \%$ of patients, the level can be resistant to bisphosphonate therapy ${ }^{58}$ with a relatively short duration (2-3 months) of serum calcium level control. Our patient's calcium level normalized with intravenous bisphosphonate and isotonic saline; however, her PTH-rP level remained elevated (34 pmol/L) while her intact PTH level remained suppressed $(1.4 \mathrm{pg} / \mathrm{mL})$ for 2 months into her hypercalcemia diagnosis and treatment. Serum PTH-rP levels can be considered to be a tumor marker and may indicate treatment response or disease progression. ${ }^{59} \mathrm{PTH}-$
$\mathrm{rP}$ has an $\mathrm{N}$-terminal and a C-terminal, which can be utilized as clinically useful assays. While both assays are elevated in patients with humoral hypercalcemia of malignancy, C-terminal PTH-rP depends on glomerular filtration and accumulates in patients with renal failure, while $\mathrm{N}$-terminal PTH-rP remains low or undetectable. ${ }^{60}$ Our patient had normal renal function. Her elevated but stable PTH-rP level, despite normalization of hypercalcemia, could be indicative of bisphosphonate treatment and stabilization of her disease by the early effect of immunotherapy and octreotide.

\section{Outcome of the Case}

Our patient, currently 6 months out from her original diagnosis, is being treated with monthly intramuscular injections of octreotide (Sandostatin LAR $30 \mathrm{mg}$ ) and intravenous pembrolizumab $200 \mathrm{mg}$ every 3 weeks. She is currently asymptomatic and tolerating her treatments well. She will be evaluated for further noncurative debulking surgery and/or liver-directed ablative therapy depending on the tumor response to systemic treatment.

For full reference list, visit:

cancernetwork.com/NET_casestudy

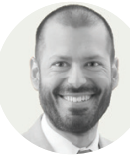

\section{Merani}

is an assistant professor, transplantation surgery, University of Nebraska Medical Center.

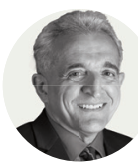

\section{Copur}

is a medical oncologist/ hematologist at Morrison Cancer Center, Mary Lanning Healthcare in Hastings, Nebraska, and is a professor at the University of Nebraska Medical Center Adjunct Faculty. He is also an Editorial Advisory Board member at ONCOLOGY ${ }^{\circledR}$.

\section{Vargas}

is an assistant professor, transplantation surgery, University of Nebraska.

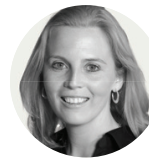

\section{Cushman-Vokoun}

is the medical director of the Molecular Diagnostics Laboratory at University of Nebraska Medical Center, and associate professor at the University of Nebraska Medical Center Department of Pathology and Microbiology, with research interest in the molecular pathology and testing of Glbased malignancies.

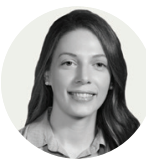

\section{Wedel}

is a staff pathologist at Mary Lanning Healthcare.

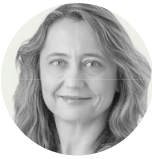

\section{Drincic}

is professor of medicine, Division of Diabetes, Endocrinology and Metabolism at the University of Nebraska Medical Center and medical director, Diabetes and Endocrinology Center. 


\section{CONTINUING MEDICAL EDUCATION (CME)}

\section{Beyond PD-1 Blockade: Novel Checkpoint Inhibitors}

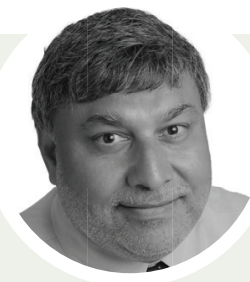

FACULTY

Naiyer A. Rizvi, MD

Price Family Professor of Medicine Director, Thoracic Oncology Co-Director of Cancer Immunotherapy Department of Medicine Division of Hematology and Oncology Columbia University Medical Center Research Director of the Price Family Comprehensive Center for Chest Care New York Presbyterian Hospital New York, NY

This activity was written by $P E R^{\circledR}$ editorial staff under faculty guidance and review. The Q\&A portion of the activity was transcribed from a recorded interview with the faculty and edited by faculty and PER ${ }^{\circledR}$ editorial staff for clarity.

\section{CIME PROVIDER CONTACT} INFORIMATION

Physicians' Education Resource ${ }^{\circledR}$, LLC 2 Clarke Drive, Suite 110

Cranbury, NJ 08512 Toll-Free: 888-949-0045

Local: 609-378-3701

Fax: 609-257-0705

info@gotoper.com

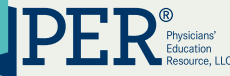

gotoper.com

\section{LEARNING OBJECTIVES}

Upon successful completion of this activity, you should be better prepared to:

- Describe mechanisms of intrinsic and acquired resistance to PD-1/PD-L1 blockade

- Explain mechanisms of action of novel and emerging checkpoint inhibitors

RELEASE DATE: December 1, 2020

EXPIRATION DATE: December 1, 2021

\section{INSTRUCTIONS FOR PARTICIPATION / HOW TO RECEIVE CREDIT}

1. Read this activity in its entirety.

2. Go to https://www.gotoper.com/go/onc20-pd1 to access and complete the posttest.

3. Answer the evaluation questions.

4. Request credit using the drop-down menu.

You may immediately download your certificate.

FACULTY, STAFF, AND PLANNERS' DISCLOSURES

In accordance with ACCME Guidelines, PER ${ }^{\circledast}$ has identified and resolved all COl for faculty, staff, and planners prior to the start of this activity by using a multistep process.

Disclosures: Naiyer A. Rizvi, MD, has no relevant financial relationships with commercial interests.

The staff of PER ${ }^{\circledast}$ have no relevant financial relationships with commercial interests to disclose.

\section{OFF-LABEL DISCLOSURE AND DISCLAIMER}

This activity may or may not discuss investigational, unapproved, or off-label use of drugs. Learners are advised to consult prescribing information for any products discussed. The information provided in this activity is for accredited continuing education purposes only and is not meant to substitute for the independent clinical judgment of a healthcare professional relative to diagnostic, treatment, or management options for a specific patient's medical condition. The opinions expressed in the content are solely those of the individual faculty members, and do not reflect those of PER ${ }^{\circledast}$ or any of the companies that provided commercial support for this activity.

This activity is funded by PER ${ }^{\circledast}$.

\section{ACCREDITATION/CREDIT DESIGNATION}

Physicians' Education Resource ${ }^{\circledast}$, LLC, is accredited by the Accreditation Council for Continuing Medical Education (ACCME) to provide continuing medical education for physicians. Physicians' Education Resource ${ }^{\circledR}$, LLC, designates this enduring material for a maximum of 0.5 AMA PRA Category 1 $\mathrm{Credits}^{\mathrm{T}}$. Physicians should claim only the credit commensurate with the extent of their participation in the activity. 
S ince the initial FDA approval of the class of agents known as immune checkpoint inhibitors, their indications and usage have expanded to include the treatment of numerous tumor types, such as bladder, prostate, lung, and colorectal cancers. ${ }^{1}$ Immune checkpoint inhibitors carry out their actions by blocking the interactions in the PD-1 pathway, an inhibiting regulatory pathway that normally suppresses T-cell immune response, or the cytotoxic T-lymphocyte associated antigen 4 (CTLA-4) pathway. By blocking these pathways, immune checkpoint inhibitors allow activated $\mathrm{T}$ cells to effectively recognize and attack tumor cells. Immune checkpoint inhibitors that are currently FDA approved ${ }^{2}$ are listed in Table 1.

As understanding of immune checkpoint mechanisms has improved in recent years, potential targets, for which novel therapies may be developed, continue to be identified. These agents are being evaluated for their potential to be used as monotherapy, in combination with currently available immune checkpoint therapies, and across several tumor types, thereby expanding the population of patients who may benefit from these treatments. ${ }^{3}$

Naiyer A. Rizvi, MD, reviews recent advances in the development of novel immune checkpoint inhibition therapies and their potential integration into the treatment landscape of numerous cancer subtypes to improve outcomes in these patients across lines of care.
Q:

\section{What is the} mechanism of action of currently available immune checkpoint inhibitors?

RIZVI: In terms of current checkpoint inhibitors, the ones that are approved target the PD-1/PD-L1 pathway. ${ }^{1}$ PD-1 is a checkpoint protein on T cells. ${ }^{4}$ It normally acts as an off switch to keep $\mathrm{T}$ cells from attacking other cells in the body. Cancers normally are recognized as foreign to the body, and your $\mathrm{T}$ cells should normally attack those foreign cells and eradicate them. However, through various mechanisms of action, the tumor cells can evade the immune system, often by upregulation of the PD-1 pathway, which allows for inhibition of $\mathrm{T}$ cells, resulting in the immune system not recognizing cancer cells as foreign.

The other pathway that has an approved checkpoint inhibitor acts on CTLA-4, which is expressed on regulatory cells. ${ }^{5}$ It is also an off switch that is involved in suppressing the immune response to cancer.

\section{What are the major mechanisms of tumor resistance to immune checkpoint inhibitor therapy?}

RIZVI: Immune checkpoint inhibitor resistance is a complicated area. Primary resistance would include scenarios in which a PD-1 pathway inhibitor is administered either alone or in combination with a CTLA-4 inhibitor and no response occurs, and there is a long list of mechanisms

\section{TABLE 1. FDA-Approved Immune Checkpoint}

\section{Inhibitors ${ }^{2}$}

\begin{tabular}{ll}
\hline Target & \multicolumn{1}{l}{ Immune checkpoint inhibitor } \\
\cline { 2 - 2 } PD-1 & Pembrolizumab \\
\hline \multirow{2}{*}{ PD-L1 } & Atezolizumab \\
\cline { 2 - 2 } & Avelumab \\
\cline { 2 - 2 } & Durvalumab \\
\hline CTLA-4 & Ipilimumab \\
\hline
\end{tabular}

CTLA-4, cytotoxic T-lymphocyte associated antigen 4; PD-1, programmed cell death protein 1; PD-L1, programmed death ligand 1.

for why that may occur. ${ }^{6}$ The most common reason is that there are not enough mutations within the tumor. There is clearly an association between high tumor mutational burden (TMB) and response to these therapies. In tumor types with very few mutations, there are less mutations to activate the immune system. In tumors with a high number of mutations, such as high microsatellite instability (MSI-H) colorectal cancer, in which DNA repair mechanisms are deficient, the tumor accumulates hundreds of thousands of mutations and those are seen as foreign to the immune system, which is being suppressed by the PD-1 pathway and other mechanisms. ${ }^{7}$ In this case, when you give the immune checkpoint inhibitor, you would turn the immune system on against these hundreds of thousands of mutations.

Certainly, the level of PD-L1 expression is also important. In tumors with low PD-L1 expression, patients are less likely to respond than in those with high PD-L1 expres- sion. ${ }^{7}$ There are several factors within the tumor microenvironment that can contribute to resistance. Pancreatic cancer, for example, has a very fibrotic, difficult-to-penetrate tumor microenvironment. Other tumors may have a high number of tumor regulatory T cells or myeloid suppressor cells. Other pathways include immunosuppressive molecules, such as IDO, arginase, glutaminase, that can be invoked, which all contribute to primary resistance. ${ }^{6,7}$

Acquired resistance is a little bit more common. This describes a situation where a patient may initially respond to a checkpoint inhibitor for a period of time and then progress, which is probably due to the tumor developing resistance mechanisms that may include subclones, which are less immunogenic. It can include tumors that develop a loss of antigens that are being presented. Or it can be from alterations in tumor antigen processing machinery, such as the JAK pathway or beta2 microglobulin. ${ }^{8}$ We know less about acquired resistance 
because it can be so variable, as it depends on the patient and the tumor type.

\section{What are the \\ - major mechanisms underlying novel immune checkpoint therapies in development?}

RIZVI: When we first started using PD-1 inhibitors and CTLA-4 inhibitors over 10 years ago, there was a lot of excitement about developing novel checkpoint agents. While both PD-1 and CTLA-4 are checkpoints that turn off the $\mathrm{T}$ cells, there are also a number of checkpoints that turn on the immune system. ${ }^{3}$ And this is the balance that occurs in healthy individuals. So when you get an infection, the agonist checkpoints turn on to activate $T$ cells, and then once you clear the infection, the inhibitory checkpoints turn on, such as PD-1, CTLA-4, to turn off the immune system to avoid rampant inflammation once you cleared the infection. So, I think that we're all very excited about many of these new potential targets, both new ways to block the inhibitory checkpoints but also ways to turn on the activating T cells.

Beyond PD-1 and CTLA-4 pathways, many of the early studies are looking at OX40, LAG-3, and others. ${ }^{9}$ It has been a bit challenging to make a material improvement beyond PD-1 and CTLA-4. I think that the challenge has been around patient selection. Many of these trials were done in the resistance setting, whereas more of these trials now are being looked at in the immunotherapy-naïve setting to say, "Okay, PD-1, CTLA-4 works this much in this type of tumor. Can we improve it by giving combination with one of these novel agents?" Current immune checkpoint inhibition pathways, especially PD- 1 , are certainly the backbone of a lot of these therapies, similarly to where platinum may have been the backbone chemotherapy agent for many types of cancers.

\section{What are the key - data from clinical} trials evaluating emerging novel immune checkpoint inhibition agents?

RIZVI: Some of the emerging data with some of these newer checkpoint pathways are looking more promising. TIM-3 is another co-inhibitory receptor that is expressed on T cells, FOXP3-positive regulatory $\mathrm{T}$ cells, and many immune cells, such as macrophages or dendritic cells. ${ }^{10}$ Some of the early data in lung cancer show that there was about $15 \%$ to $20 \%$ activity with TIM-3 inhibitors in the acquired-resistance setting. ${ }^{11}$ T-cell immunoreceptor with Ig and ITIM domains (TIGIT) has been showing some very encouraging data. It is an inhibitory immunoreceptor on lymphocytes involved in the PVR-nectin family and associated with different T-cell exhaustion phenotypes. ${ }^{12}$ TIGIT can enhance antitumor response through its role as a ligand-receptor competitor for CD226. Some of the data presented earlier this year showed a high level of activity in first-line lung cancer. ${ }^{13}$

\section{TABLE २. Select Investigational Immune} Checkpoint Inhibition Agents

\begin{tabular}{ll}
\hline Target & $\begin{array}{l}\text { Therapeutic agent } \\
\text { Eftilagimod alpha }\end{array}$ \\
LAG-3 & $\begin{array}{l}\text { (IMP321) } \\
\text { Relatlimab } \\
\text { (BMS-986016) }\end{array}$ \\
\hline TIM-3 & $\begin{array}{l}\text { Cobolimab }^{19} \\
\text { (TSR-022) }\end{array}$ \\
\cline { 2 - 2 } & $\begin{array}{l}\text { Sabatolimab } \\
\text { (MBG453) }\end{array}$ \\
\hline OX-40 & PF-04518600 \\
\hline TIGIT & Tiragolumab \\
\hline
\end{tabular}

LAG-3, lymphocyte activation gene 3; TIGIT, T-cell immunoreceptor with immunoglobulin and ITIM domain; TIM-3, T-cell immunoglobulin and mucin domain-3.

Interestingly, the patients for whom PD-1 monotherapy with pembrolizumab is approved, the greater than or equal to $50 \%$ expression group seem to have the greatest benefit. So, even though PD-1/PD-L1 inhibitors seem to be active in high PD-L1 expression, the response rates are still in the $40 \%$ range, and many of them are not durable. In the preliminary data, inhibiting TIGIT with PD-1, the response rates were more in the $60 \%$ range. $^{13}$

These agents have entered phase 3 trials, both in nonsmall cell and small cell lung cancer, which is exciting. Some of the compounds which are showing promise in very early data now include the inhibitor of immunoglobulin-like transcript 4 (ILT4), an immunosuppressive molecule expressed in myeloid cells predominately. ${ }^{14}$ Some of the early data shows activity broadly in a number of tumor types with ILT4 inhibitors. Some of the data with NKG2 inhibitors, particularly in head and neck cancer, has also shown promise. ${ }^{15}$ Blocking NKG2 is relevant for activation of the innate immune pathway, which includes the natural killer pathway. And data here has shown very good activity in head and neck cancers.

Finally, some new bispecifics are demonstrating activity, such as PD-1/CTLA-4 bispecifics, TGF-beta pathway bispecifics (which is not a checkpoint per se, but beyond novel immune checkpoints). We are also trying to inhibit other immunosuppressive pathways within the tumor microenvironment that can limit the effectiveness of PD-1. ${ }^{16}$

\section{What are some of - the major adverse events observed with novel emerging immune checkpoint agents?} RIZVI: When we think about toxicities with the immune 
checkpoint inhibitors, obviously PD-1, CTLA-4 have significant autoimmune toxicities, particularly when given in combination. In preclinical models, if you knock out PD-1 or CTLA-4, you get limitations in development; worse with CTLA-4 than PD-1. But many of these other novel immune checkpoints, when you knock them out, you do not see that much happen to the animals, suggesting they may be less critical in terms of the immune system pathways. ${ }^{3}$

So we are not really seeing a compounding of toxicities with many of these immune checkpoint inhibitors. Certainly, in some of the early data with super-agonists that really revved up the immune system too much, we saw a fair amount of toxicity, including pulmonary toxicity, capillary-release syndrome, and things like that. But using some of the checkpoints that we are studying more recently in the clinic, we have not seen much in the way of dose-limiting toxicity, aside from some mild toxicities that you might expect with PD-1, CTLA-4, but nothing dramatic. And so they do lend themselves well to combination with PD-1 or CTLA-4.

\section{What is the role 1. of biomarkers in predicting which class of novel emerging immune checkpoint agents is used?}

RIZVI: In terms of biomarkers, we really have not identified subsets that indicate whether they should get TIGIT or TIM-3 or LAG-3, just like we really have no biomarker for CTLA-4. Certainly, for some of these compounds like TIGIT, if you have high levels of PD-L1 expression, you often have higher levels of TIGIT, so they kind of go hand in hand quite often. And so certainly for TIGIT, PD-L1 expression may be a relevant biomarker. And so, if we focus on PDL1-high tumors, perhaps, that may be sort of double-positive for PD-L1, as well as TIGIT expression, and there could be a biomarker there. In general, we do not really have a good sense of biomarkers for some of the novel immune checkpoint inhibitors in development, but it is certainly a work in progress.

\section{F. For which tumor types do emerging immune checkpoint agents appear to be most effective?}

RIZVI: The TIGIT seems to be enhancing PD-1 pathway inhibition, particularly in non-small cell lung cancer. ${ }^{22}$ The TIM-3 agent is also showing activity in lung cancer. ${ }^{20}$ The inhibitors of NKG2A are showing promise in head and neck cancer. ${ }^{23}$ ILT4 is seeing activity in a broad range of tumor types, but it's much earlier in development. LAG-3 is also showing broad activity early on. ${ }^{17,18}$ The answer is probably that where you see modest activity for the PD-1 pathway, such as in head and neck, lung, gastric, bladder, that may be where the opportunity is. Tumors with low TMB, low T cell infiltration, I am not sure that it is impacting those tumors.

\section{What are the major challenges in the development of novel immune checkpoint therapies?}

RIZVI: I think it is challenging to integrate. We have not had much advancement over the last 10 to 12 years. We have not been able to get FDA approval of immune checkpoint inhibitors beyond the PD-1 pathway and CTLA-4. We are seeing more of the next-generation immune checkpoint inhibitors with new targets showing promise, and many of them are in Phase 3 trials currently, so hopefully these will read out soon and we'll have better combinations in the future.

\section{KEY REFERENCES}

1. Ribas A, Wolchok JD.

Cancer immunotherapy using checkpoint blockade. Science. 2018;359(6382):1350-1355. doi:10.1126/science.aar4060

3. Qin S, Xu L, Yi M, Yu S, Wu K, Luo $S$. Novel immune checkpoint targets: moving beyond PD-1 and CTLA-4. Mol Cancer 2019;18(1):155. doi:10.1186/ s12943-019-1091-2

9. Marin-Acevedo JA, Dholaria B, Soyano AE, Knutson KL, Chumsri

S, Lou Y. Next generation of immune checkpoint therapy in cancer: new developments and challenges. J Hematol Oncol. 2018;11(1):39. doi:10.1186/ s13045-018-0582-8

12. Chauvin JM, Zarour HM. TIGIT in cancer immunotherapy. $\mathrm{J}$ Immunother Cancer. 2020;8(2):e000957. doi:10.1136/ jitc-2020-000957

For full reference list, visit https://www.gotoper.com/go/ onc20-pd1 


\section{Medical Economics

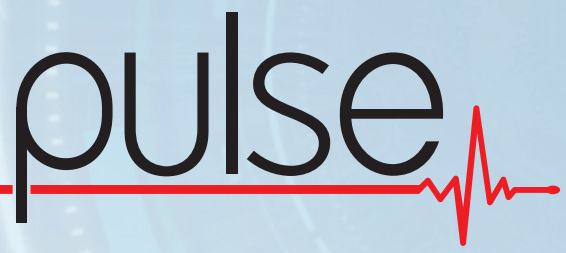

\section{Succeed as a caregiver and businessperson.}

Access thought provoking video interviews with industry experts on managing your medical practice.

» How physicians can protect personal assets from liability

»Tips to get your practice finances back on track

» Major coding changes for 2021, explained

»The latest on diabetes management: Treatment plans, therapies, tech and more

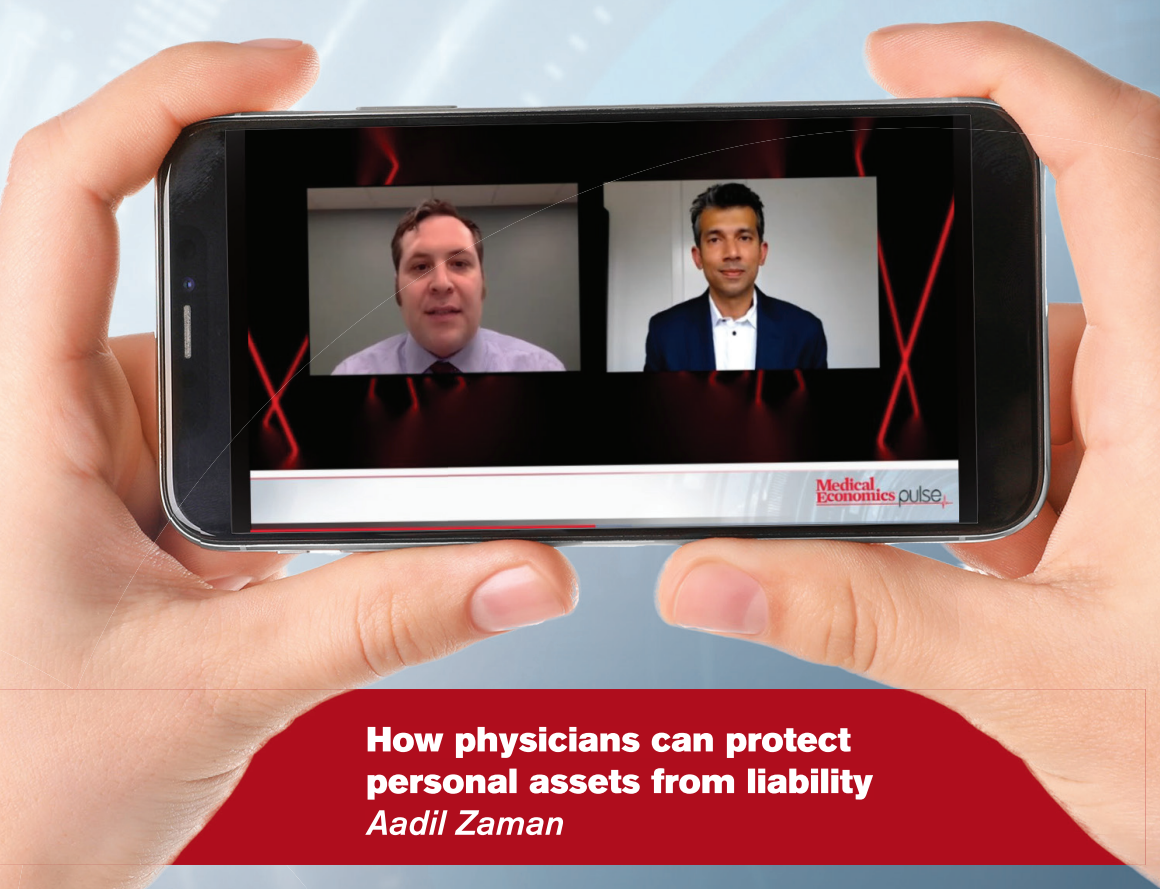

View the video series!

www.medicaleconomics.com/medical-economics-pulse

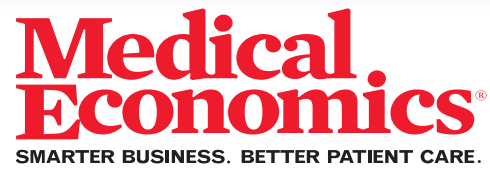



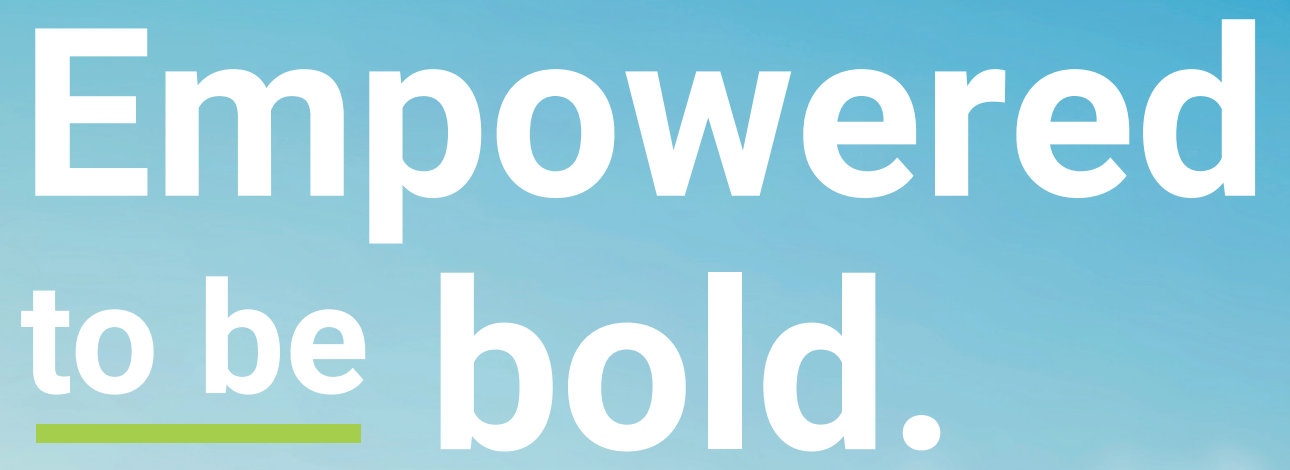

We're mastering the art of
patient-focused treatment

Our robust investigational pipeline of novel small molecule, biologic, and cellular therapies is focused on both improving outcomes and improving the cancer treatment experience for patients around the world.

Be the first to learn about the latest technology in metastatic breast cancer treatment and beyond at AthenexOncology.com

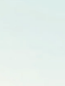




\section{Neutropenia (cont'd)}

Monitor complete blood cell counts periodically during treatment. Consider the use of antibiotics and antiviral prophylaxis during treatment. Monitor patients with neutropenia for signs of infection. In case of grade 4 neutropenia, delay SARCLISA dose until neutrophil count recovery to at least $1.0 \times 10^{\circ} / \mathrm{L}$, and provide supportive care with growth factors, according to institutional guidelines. No dose reductions of SARCLISA are recommended.

\section{Second Primary Malignancies}

Second primary malignancies were reported in 3.9\% of patients in the SARCLISA, pomalidomide, and dexamethasone (Isa-Pd) arm and in $0.7 \%$ of patients in the pomalidomide and dexamethasone (Pd) arm, and consisted of skin squamous cell carcinoma $(2.6 \%$ of patients in the Isa-Pd arm and in $0.7 \%$ of patients in the Pd arm), breast angiosarcoma (0.7\% of patients in the Isa-Pd arm), and myelodysplastic syndrome $(0.7 \%$ of patients in the Isa-Pd arm). With the exception of the patient with myelodysplastic syndrome, patients were able to continue SARCLISA treatment. Monitor patients for the development of second primary malignancies.

\section{Laboratory Test Interference \\ Interference with Serological Testing (Indirect \\ Antiglobulin Test)}

SARCLISA binds to CD38 on red blood cells (RBCs) and may result in a false positive indirect antiglobulin test (indirect Coombs test). In ICARIA-multiple myeloma (MM), the indirect antiglobulin test was positive during SARCLISA treatment in $67.7 \%$ of the tested patients. In patients with a positive indirect antiglobulin test, blood transfusions were administered without evidence of hemolysis. $A B O / R h D$ typing was not affected by SARCLISA treatment. Before the first SARCLISA infusion, conduct blood type and screen tests on SARCLISAtreated patients. Consider phenotyping prior to starting SARCLISA treatment. If treatment with SARCLISA has already started, inform the blood bank that the patient is receiving SARCLISA and SARCLISA interference with blood compatibility testing can be resolved using dithiothreitol-treated RBCs. If an emergency transfusion is required, non-cross-matched $A B O / R h D$-compatible RBCs can be given as per local blood bank practices.

\section{Interference with Serum Protein Electrophoresis and Immunofixation Tests}

SARCLISA is an lgG kappa monoclonal antibody that can be incidentally detected on both serum protein electrophoresis and immunofixation assays used for the clinical monitoring of endogenous M-protein. This interference can impact the accuracy of the determination of complete response in some patients with IgG kappa myeloma protein.

\section{Embryo-Fetal Toxicity}

Based on the mechanism of action, SARCLISA can cause fetal harm when administered to a pregnant woman. SARCLISA may cause fetal immune cell depletion and decreased bone density. Advise pregnant women of the potential risk to a fetus. Advise females with reproductive potential to use an effective method of contraception during treatment with SARCLISA and for at least 5 months after the last dose. The combination of SARCLISA with pomalidomide is contraindicated in pregnant women because pomalidomide may cause birth defects and death of the unborn child. Refer to the pomalidomide prescribing information on use during pregnancy.

\section{ADVERSE REACTIONS}

The most common adverse reactions ( $\geq 20 \%)$ were neutropenia (laboratory abnormality, $96 \%$ Isa-Pd vs $92 \%$ $\mathrm{Pd}$ ), infusion-related reactions ( $38 \% \mathrm{Isa}-\mathrm{Pd}$ vs $0 \% \mathrm{Pd}$ ), pneumonia ( $31 \% \mathrm{Isa}-\mathrm{Pd}$ vs $23 \% \mathrm{Pd}$ ), upper respiratory tract infection ( $57 \%$ Isa-Pd vs $42 \% \mathrm{Pd}$ ), and diarrhea ( $26 \%$ with Isa-Pd vs $19 \%$ Pd). Serious adverse reactions occurred in $62 \%$ of patients receiving SARCLISA. Serious adverse reactions in $>5 \%$ of patients who received Isa-Pd included pneumonia (26\%), upper respiratory tract infections (7\%), and febrile neutropenia (7\%). Fatal adverse reactions occurred in $11 \%$ of patients (those that occurred in more than $1 \%$ of patients were pneumonia and other infections [3\%]).

\section{USE IN SPECIAL POPULATIONS}

Because of the potential for serious adverse reactions in the breastfed child from isatuximab-irfc administered in combination with $\mathrm{Pd}$, advise lactating women not to breastfeed during treatment with SARCLISA.

\section{Please see Important Safety Information throughout, and Brief Summary of the full Prescribing Information.}

\footnotetext{
References: 1. SARCLISA [prescribing information]. Bridgewater, NJ: sanofi-aventis U.S. LLC. 2. Attal M, Richardson PG, Rajkumar SV, et al; on behalf of the ICARIAMM study group. Isatuximab plus pomalidomide and low-dose dexamethasone versus pomalidomide and low-dose dexamethasone in patients with relapsed and refractory multiple myeloma (ICARIA-MM): a randomised, multicentre, open-label, phase 3 study. Lancet. 2019;394(10214):2096-2107. 3. Data on file. sanofi-aventis U.S. LLC. 4. Referenced with permission from the NCCN Clinical Practice Guidelines in Oncology (NCCN Guidelines ${ }^{\circledR}$ ) for Multiple Myeloma V.2.2021. ๑ National Comprehensive Cancer Network, Inc. 2020. All rights reserved. Accessed September 9, 2020. To view the most recent and complete version of the guideline, go online to NCCN.org.
} 
IN THE TREATMENT OF RELAPSED REFRACTORY

MULTIPLE MYELOMA IN COMBINATION WITH Pd

\title{
Achieve Greater Outcomes for Your Patients
}

\author{
The first phase 3 trial of an anti-CD38 mAb in \\ combination with $\mathrm{Pd}$ vs $\mathrm{Pd}$ alone
}

SARCLISA + Pd

$(n=154)$

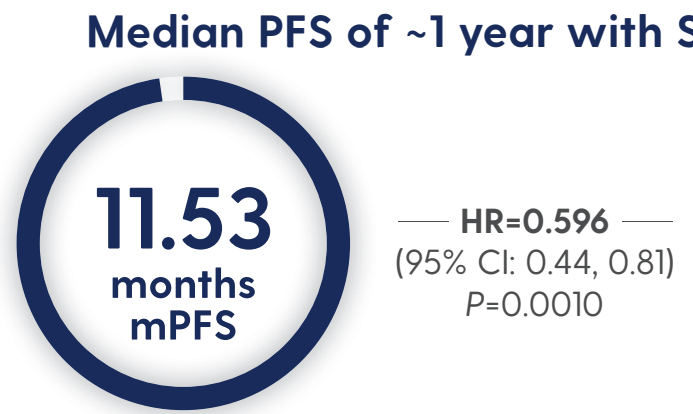

(95\% Cl: 8.94, 13.9) $\begin{aligned} & \text { HR }=0.596 \\ &(95 \% \mathrm{Cl}: 0.44,0.81)\end{aligned}$

$P=0.0010$

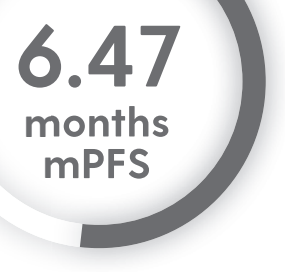

Pd

$(n=153)$

A significant increase in responses shown with SARCLISA + Pd ${ }^{1 *}$

\begin{tabular}{|lcl|}
\hline \multicolumn{2}{l}{ SARCLISA + Pd $(n=154)$} & $P d(n=153)$ \\
\hline $60.4^{\%}$ ORR & $P<0.0001$ & $35.3^{\%}$ ORR \\
\hline $31.8 \% \geq V G P R$ & $\sim 4 \times$ increase & $8.5 \% \geq V G P R$ \\
\hline 35 days & $\begin{array}{c}\text { Median time to first response } \\
\text { among responders }\end{array}$ & $\mathbf{5 8}$ days
\end{tabular}

*ORR included sCR, CR, VGPR, and PR. ORR: SARCLISA + Pd (95\% Cl: 52.2\%, 68.2\%), Pd (95\% Cl: $27.8 \%, 43.4 \%)$.

- $7 \%$ of patients receiving SARCLISA + Pd permanently discontinued treatment due to adverse reactions ${ }^{1}$

- Infusion time decreases to 75 minutes starting after the second infusion in the absence of IRRs'

IRR=infusion-related reaction; $\mathrm{mPFS}=$ median progression-free survival.

\section{Indication}

SARCLISA (isatuximab-irfc) is indicated, in combination with pomalidomide and dexamethasone, for the treatment of adult patients with multiple myeloma who have received at least two prior therapies including lenalidomide and a proteasome inhibitor.

\section{Important Safety Information}

\section{CONTRAINDICATIONS}

SARCLISA is contraindicated in patients with severe hypersensitivity to isatuximab-irfc or to any of its excipients.

\section{WARNINGS AND PRECAUTIONS}

\section{Infusion-Related Reactions}

Infusion-related reactions (IRRs) have been observed in 39\% of patients treated with SARCLISA. All IRRs started during the first SARCLISA infusion and resolved on the same day in $98 \%$ of the cases. The most common symptoms of an IRR included dyspnea, cough, chills, and nausea. The most common severe signs and symptoms included hypertension and dyspnea.

Please see Important Safety Information throughout, and accompanying Brief Summary of the full Prescribing Information. 